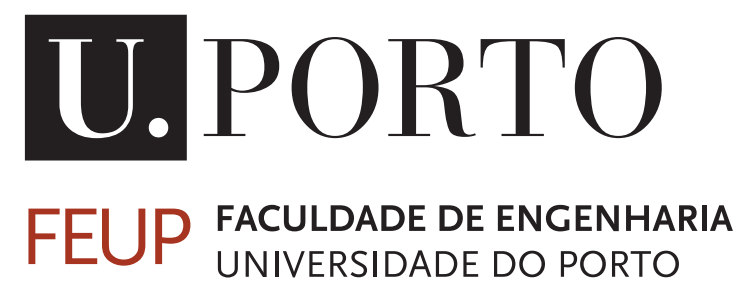

\title{
Automatic Macular Edema Identification and Characterization Using OCT Images
}

Fátima Gabriela dos Santos Samagaio

\author{
DISSERTATION
}

Master in Biomedical Engineering

Supervisor: Joaquim Gabriel Mendes, PhD (FEUP)

Supervisor: Jorge Novo Buján, PhD (FIC, Spain) 
(C) Gabriela Samagaio, 2018 


\section{Resumo}

A Tomografia de Coerência Óptica (TCO) consiste numa técnica de imagem médica não invasiva e ausente do contacto directo com o paciente que avalia in vivo a histopatologia do tecido. Esta tem sido amplamente utilizada no diagnóstico médico para identificar diversas patologias, não só associadas ao órgão em estudo no presente projecto, mas também a outras doenças em geral.

A Retinopatia Diabética (RD) e a Degeneração Macular Relacionada à Idade (DMRI) são atualmente consideradas como duas das principais doenças oculares relacionadas com a perda de visão nos países desenvolvidos. Estas doenças têm em comum o acúmulo de líquido dentro do tecido retiniano, formando o Edema Macular (EM). A detecção e caracterização da acumulação de líquido intra-retiniano na mácula constitui uma questão oftalmológica crucial, dado que este fornece informações úteis na identificação e no diagnóstico dos diferentes tipos de EM, indicando consequentemente a gravidade da doença. Estes tipos de edemas da retina são clinicamente definidos, segundo a classificação de referência do campo, como: Descolamento Seroso Exsudativo da Retina (DSER), Edema Macular Difuso (EMD) e Edema Macular Cistoide (EMC).

O presente trabalho visa o desenvolvimento de um sistema automático capaz de detectar e caracterizar os três tipos de EM usando imagens TCO. Este sistema de Diagnóstico Assistido por Computador (DAC) usará diferentes estratégias as quais combinam técnicas de processamento de imagem e de aprendizagem máquina com o conhecimento clínico. No caso do reconhecimento dos edemas DSER e EMC, espera-se a aplicação de abordagens com base nos diferentes limiares de intensidades característicos destes edemas, enquanto que para os EMD, dada a sua complexidade e aparência regional, deve ser aplicada uma estratégia de aprendizagem para explorar propriedades características dessa região.

Esta solução permitirá assegurar a redução de custos relacionados com operações cirúrgicas e tratamento clínico aumentando assim a qualidade do diagnóstico, reduzindo a subjectividade inter-especialistas culminando na melhora da qualidade de vida do paciente. A produtividade e eficiência dos especialistas irá igualmente aumentar dado que esta solução irá o seu trabalho será facilitado.

Palavras-Chaves: Diagnóstico Assistido por Computador, Tomografia de Coerência Óptica, Edema Macular, Retinopatia Diabética, Degeneração Macular Relacionada à Idade 


\section{Abstract}

The Optical Coherence Tomography (OCT) consists of a non-invasive and contactless medical image technique that evaluates in vivo the histopathology of the tissue. It has been widely used in medical diagnosis to identify a large range of pathologies, not only associated to the organ under the present study but also with other diseases in general.

The diabetic retinopathy (DR) and the Age-related Macular Degeneration (AMD) are considered the two major eye diseases that are currently related with the vision loss in the developed countries. These diseases have in common the fluid accumulation within the retinal tissue round the macular region, forming the Macular Edema (ME). The detection and characterization of the intraretinal fluid accumulation constitutes a crucial ophthalmological issue as it provides useful information for the identification and diagnosis of the different types of ME, consequently indicating the severity of the disease. These types of retinal edemas are clinically defined, by the reference classification of the field, as: Serous Retinal Detachment (SRD), Diffuse Retinal Thickening (DRT) and Cystoid Macular Edema (CME).

Therefore, the proposed project aims to develop an automatic system capable of detecting and characterizing these three types of ME using OCT images. Thus, the development of a ComputerAided Diagnosis (CAD) system is expected to detect each type of ME using different strategies, which combine image processing and learning techniques with clinical knowledge. In the case of the recognition of SRD and CME edemas, the application of multilevel image thresholding approaches is expected, whereas for the DRT edemas, given their complexity and regional appearance, a learning strategy should be applied in order to exploit representative properties from these regions.

This solution will ensure cost reductions related to surgical operations and clinical treatment by increasing the quality of the diagnosis. Moreover, this system will allow the reduction of the subjectivity among specialists, consequently leading to an improvement of the quality of life of the patients under clinical evaluation. Also, the productivity and efficiency of the specialists will be increased since the proposed project will facilitate and simplify their work.

Key-Words: Computer-Aided Diagnosis, Optical Coherence Tomography, Macular Edema, Diabetic Retinopathy, Age-related Macular Degeneration 


\section{Agradecimentos}

Em primeiro lugar gostaria de dedicar um sentido agradecimento ao Grupo de Investigação VARPA, por me ter dado, pela segunda vez consecutiva, a oportunidade de integrar a equipa e desta forma, poder processeguir com o projeto previamente iniciado. Se hoje sou a pessoa e a profissional que sou, devo em grande parte a esta equipa que desde o primeiro dia me acompanha e me orienta.

$\mathrm{Na}$ bagagem levo o conjunto de ferramentas adquiridas durante este período para assim construir o meu percurso para o sucesso. Aprendi que sem sacrifício e esforço nada se consegue, e toda esta equipa é prova disso. O caminho pode ter sido difícil, às vezes até demais. Mas todos souberam manter a confiança uns nos outros e nunca desistiram ou afastaram o olhar da meta. Hoje colhemos os frutos dessa dedicação e agora desfrutamos do triunfo.

Como membros desta equipa, gostaria de prestar um especial agradecimento ao meu supervisor da dissertação, Professor Doutor Jorge Novo, pelos ensinamentos, orientação, motivação e paciência demonstrados ao longo deste percurso. E ainda, ao doutorando Joaquim de Moura pelos princípios e valores que me transmitiu e acima de tudo pelo apoio incondicional. Considero-me um dos seus pequenos pupilos.

Seguidamente, gostaria ainda de agradecer ao Professor Doutor Joaquim Mendes não só por ter aceite o desafio de ser o meu orientador, como também por ter acompanhado a minha evolução ao longo destes 3 anos académicos.

Em especial gostaria ainda de agradecer à minha família, em particular à pessoa mais importante na minha vida, a minha mãe. Obrigada por teres acreditado sempre em mim, por me teres transmitido o teu orgulho e força inesgotável. Se hoje venci muitas balhatas e atingi muitos dos meus objetivos foi pelo teu apoio incondicional, mãe.

Aos meus verdadeiros amigos(as), um sincero obrigada por "aturarem" mesmo à distância as minhas loucuras que só vocês conhecem, mas igualmente por me ampararem a queda nos momentos de maior aflição. "Ai miei piccoli", obrigada pela experiência inesquecível, formamos "pior" família possível, tão disfuncional e louca, que mesmo já separados pela distância o nosso carinho e apoio em nada mudou. Finalmente, mas não menos importante, gostaria de agradecer à "família gym" pelo apoio inesgotável e pela boa energia transmitida diariamente.

Gabriela Samagaio 


\section{Contents}

1 Introduction 1

1.1 Motivation ...................... 1

1.2 Research Goals and Contributions . . . . . . . . . . . . . . . 3

1.3 Dissertation Outline . . . . . . . . . . . . . . . . . 3

2 Domain Description 5

2.1 The Human Eye Anatomy and Physiology . . . . . . . . . . . . . . 5 5

2.1.1 Retinal Anatomy and Physiology _............. 8

2.2 Image Extraction: Ophthalmoscopy . . . . . . . . . . . . . . . . . 13

2.2.1 Direct Opthalmoscope . . . . . . . . . . . . . . . . . . 13

2.2 .2 Indirect Opthalmoscope $\ldots \ldots \ldots \ldots \ldots$

2.3 Macular Edema (ME) Pathology . . . . . . . . . . . . . . . . . . 18

2.3.1 Description of the ME Pathology . . . . . . . . . . . . . . 18

2.4 Macular Edema Types . . . . . . . . . . . . . . . . . . . . 22

2.4.1 Serous Retinal Detachment - SRD . . . . . . . . . . . . . . . . 23

2.4.2 Diffuse Retinal Thickening - DRT . . . . . . . . . . . . . . . 24

2.4.3 Cystoid Macular Edema - CME . . . . . . . . . . . . . . 25

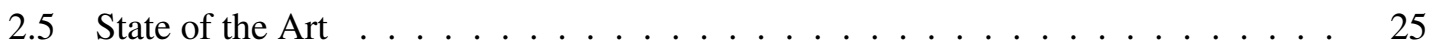

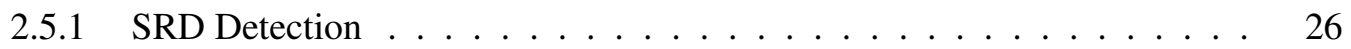

2.5.2 SRD and CME Detection . . . . . . . . . . . . . . . . 27

2.5 .3 CME Detection . . . . . . . . . . . . . . . . . . . 27

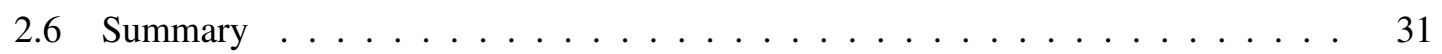

3 Methodology 33

3.1 Description of the Main Methodology Stages . . . . . . . . . . . . . . 33

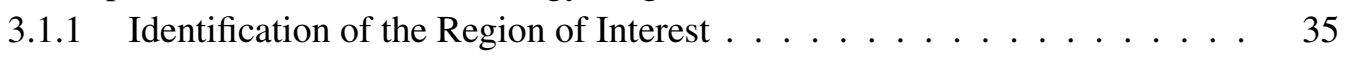

3.1.2 Retinal Layer Segmentation . . . . . . . . . . . . . . . 35

3.1.3 Identification of the Different Types of ME . . . . . . . . . . . 38

3.2 Disease Characterization . . . . . . . . . . . . . . . . . 47

3.3 Properties of the Required Software . . . . . . . . . . . . . . . 47

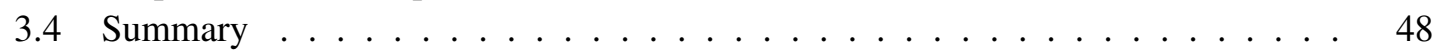

4 Results 49

5 Discussion 55

6 Conclusions 63 
A Publications 65

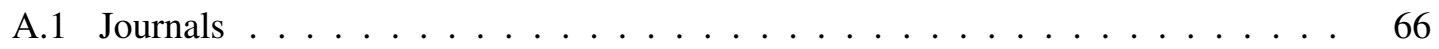

A.2 Conferences ............................ 66

References 


\section{List of Figures}

2.1 Human eye anatomy with the representation of the formation of the captured

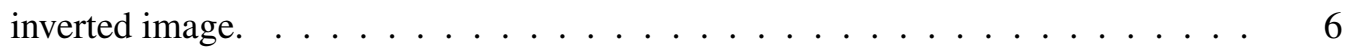

2.2 External human eye anatomy of the eye surface. . . . . . . . . . . . 7

2.3 The eye is composed of three concentric tunics. . . . . . . . . . . . 7

2.4 Scheme of the path traveled by light through the eye . . . . . . . . . . 9

2.5 Optimal wavelengths for human rod and cone photoreceptors cells. . . . . . . . . 9

2.6 Main retina structures. . . . . . . . . . . . . . . . . . . . 10

2.7 Distribution of cones and rods photoreceptors cells across the human retina. . . . 11

2.8 Example of an OCT image with the identification of the retinal layers. . . . . . 12

2.9 Example of OCT image with the inner and outer retina identification. . . . . . . 13

2.10 Direct ophthalmoscope technique. . . . . . . . . . . . . . . . 14

2.11 Modern direct ophthalmoscope. . . . . . . . . . . . . . . . . . . 14

2.12 Monocular indirect ophthalmoscope technique. . . . . . . . . . . . 15

2.13 Binocular indirect ophthalmoscope technique. . . . . . . . . . . . . . . 16

2.14 Process to capture the OCT images. . . . . . . . . . . . . . . 17

2.15 Opthalmologic test using the OCT device in a patient. . . . . . . . . . . 18

2.16 Schematic illustration of the biological mechanism forming the ME. . . . . . . . 19

2.17 Comparative scheme between a normal and pathological eye [1] . . . . . . . . 20 20

2.18 Example of OCT image with 3 types of MEs. . . . . . . . . . . 23

2.19 Example of OCT image with the presence of SRD edema. . . . . . . . . 24

2.20 Example of OCT image with the presence of DRT edema. . . . . . . . . . 24

2.21 Example of OCT image with the presence of CME edemas. . . . . . . . . . 25

3.1 Main steps of the proposed methodology for the identification and characterization of the three types of ME as SRD, DRT and CME . . . . . . . . . . . . . . 34

3.2 Example of OCT image with the identification of the ILM, OPL, ISOS and RPE

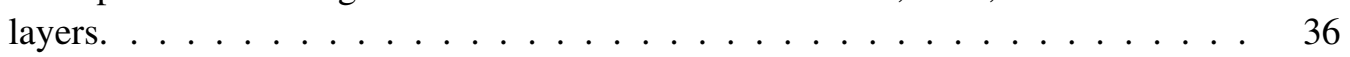

3.3 Example of OCT image with the representation of three main retinal layers ILM, ISOS and RPE. . . . . . . . . . . . . . . . . . . . . . . 36

3.4 Example of OCT image with the identification of the OPL layer by the application of the Region Growing approach. . . . . . . . . . . . . . . . . 37

3.5 Scheme for the division of the ROI in two sub-regions: inner and outer retina. . . 38

3.6 Example of OCT image with the identification of the SRD edema. . . . . . . . . 39

3.7 Example of OCT image with the presence of DRT edema. . . . . . . . . . 41

3.8 Illustration of separating two classes using SVM method. . . . . . . . . . . 43

3.9 Example of OCT image with the presence of a hard-exudate . . . . . . . . . . 44

3.10 Example of OCT image with application of the aggregation factor. . . . . . . . 44

3.11 Example of OCT image with the presence of CME edemas. . . . . . . . . 45 
3.12 Example of OCT with the presence of CMEs in the outer retina with a variable size and morphology shapes. . . . . . . . . . . . . . . 45

3.13 Example of OCT image with the presence of CMEs in the outer retina. . . . . . 4 46

4.1 Determination of the optimal aggregation factor. . . . . . . . . 53

4.2 The application of the aggregation factor unifies single candidates in a grouped "sponge-like" edema detection. . . . . . . . . . . . . . . . . 54

5.1 Comparative analysis between the performance of the original method and our proposal for the OPL layer and the outer retina identifications, using nonpathological and pathological cases. . . . . . . . . . . . 56

5.2 Example of OCT images with the OPL identification from the output of the original method and our proposal. . . . . . . . . . . . . 57

5.3 Example of OCT image with the presence of CMEs. . . . . . . . . . . 58

5.4 Example of OCT image with a non-detected CME, with an elliptical horizontal

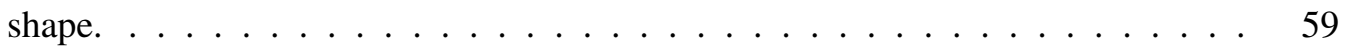

5.5 Examples of ME detections in OCT images. . . . . . . . . . . . . . 60

5.6 Example of OCT image with the detection of the three types of MEs: SRD illustrated, CME and DRT. . . . . . . . . . . . . . . . 61 


\section{List of Tables}

2.1 Comparative list of the different studies that are present in the literature for the identification of fluid regions. . . . . . . . . . . . . 30

3.1 List of the defined 18 features to identify the DRT presence. . . . . . . . 42

4.1 Obtained results for the OPL layer and outer retina identifications in nonpathological and pathological OCT images. . . . . . . . . . . . 51

4.2 Obtained results by the analyzed classifiers and the selected feature subsets. . . 5 53

4.3 Obtained results for the DRT detection using the best configurations of the Naive Bayes and SVM classifiers using the testing dataset. . . . . . . . . . . 53

4.4 F-Measures that were obtained with the tested classifiers and their corresponding optimal aggregation factors using the testing dataset. . . . . . . . . . . 54

4.5 Results of the CME detection using Precision, Recall and F-Measure. . . . . . . 54

5.1 CME detection performance comparison of state of the art approaches and the proposed method. . . . . . . . . . . . . . . . . . 61 


\section{Abbreviations}

\begin{tabular}{|c|c|}
\hline AMD & Aged-related Macular Degeneration \\
\hline BoW & Bag of Words \\
\hline CAD & Computer-Aided Design \\
\hline CME & Cystoid Macular Edema \\
\hline $\mathrm{CNN}$ & Convolutional Neural Network \\
\hline DME & Diabetic Macular Edema \\
\hline DR & Diabetic Retinopathy \\
\hline DRT & Diffuse Retinal Thickening \\
\hline ELM & External Limiting Membrane \\
\hline $\mathrm{FCN}$ & Fully Convolutional Neural Network \\
\hline FN & False Negative \\
\hline FP & False Positive \\
\hline GCL & Ganglion Cell Layer \\
\hline GMM & Gaussian Mixture Models \\
\hline HOG & Histogram Oriented Gradients \\
\hline ILM & Inner Limiting Membrane \\
\hline INL & Inner Nuclear Layer \\
\hline IPL & Inner Plexiform Layer \\
\hline ISOS & Inner Segment and Outer Segment layer \\
\hline KNN & K-Nearest Neighbors \\
\hline LBP & Local Binary Patterns \\
\hline LDA & Linear Discriminant Analysis \\
\hline MATLAB & Matrix Laboratory \\
\hline $\mathrm{ME}$ & Macular Edema \\
\hline MH & Macular Hole \\
\hline MME & Microcystic Macular Edema \\
\hline NDP & National Diabetes Program \\
\hline NFL & Nerve Fiber Layer \\
\hline OCT & Optical Coherence Tomography \\
\hline ONL & Outer Nuclear Layer \\
\hline OPL & Outer Plexiform Layer \\
\hline PAC & Principal Component Analysis \\
\hline PL & Photoreceptor Layer \\
\hline RHA & Regional Health Administration \\
\hline ROI & Region of Interest \\
\hline
\end{tabular}


RPE Retinal Pigment Ephitelium

SLD Super-Luminescent Diode

SRD Serous Retinal Detachment

SVM Support Vector Machine

TN True Negative

TP True Positive

VEGF Vascular Endothelial Growth Factor

WHO World Health Organization 


\section{Chapter 1}

\section{Introduction}

The present dissertation was proposed by the VARPA research group as a strategy to continue the previous studies started in the second semester of the previous academic year (2017/2018). This project was developed under the Erasmus+ Mobility for traineeship program, being fully developed in the Faculty of Computer Science of the University of A Coruña, Spain, in participation with a heterogeneous scientific group.

The present project aims to develop in MATLAB ${ }^{\circledR}$ a package for automatic detection and characterization of the three types of Macular Edema (ME) as Serous Retinal Detachment (SRD), Diffuse Retinal Thickening (DRT) and Cystoid Macular Edema (CME).

This chapter provides an introductory motivation for the study. Then, the research goals and the contributions of this project are briefly described. Finally, a general description of the structure of the present dissertation project is presented with a short explanation of the content addressed in each chapter.

\subsection{Motivation}

Visual impairment is a major worldwide health problem which, besides the effect on the normal vision, also carries a significant loss of quality of life and an important economic cost both to the patients and the healthcare systems. In particular, following the recent statistics from the World Health Organization (WHO), it was estimated that a patient with visual impairments has three times more chances to suffer a fall while walking, to be unemployed, to have a motor vehicle collision or even to suffer from a depressive and anxiety disorder [2]. According to the latest data, in 2017 this organization estimated that 253 million people lived with vision impairment, of which 36 million were blind and 217 million had moderate to severe vision loss [3] among working-aged adults. Additionally, in 2050, it was estimated that the number of people with visual impairment could be triplicated [4]. Thus, an early detection of the retinal diseases, a correct treatment implementation and a reliable method to predict the patient's response to therapies, represent a major necessity in the biomedical and, in particular, in the ophthalmological fields [5]. 
In the last 10 years, despite the progress that has been made in surgical techniques, cataracts still remains the leading cause of visual impairment, affecting a $47 \%$ of the population with visual problems. In particular, the ME can be related with many causes, as diabetes (typically causes the Diabetic Retinopathy (DR)), Age-related Macular Degeneration (AMD) and cataracts, which makes this eye pathology the second leading cause of vision loss [6]. ME refers to an abnormal blister of fluid in the layers over the macular region, which changes the morphology of the retinal tissue. The presence of this fluid accumulation within this sensitive-light tissue leads to a significant modification of the normal morphology, architecture and functions of this tissue (especially regarding its thickness). This visual reduction is intrinsically related with the presence of fluid in the retinal tissue, once it constitutes the light-sensitive tissue at the back of the eye. Additionally, it affects the normal function of the macula, given this is the part of the retina responsible for sharp, central and color vision, this fluid accumulation leads to the deterioration of the visual quality and, in more advanced clinical stages, it could lead to complete blindness. The more diffuse and thicker, the more severe the swelling is, significantly decreasing the visual acuity of the patient [7]. In this way, if this retinal pathology is not correctly diagnosed or treated, this pathology could become chronic, leading to an irreversible damage of the macula and a permanent vision loss [8]. Moreover, the lifespan is a factor that contributes to higher statistical values, once a considerable percentage of the population is reaching elderly ages, where the problems in the vision are more frequent. Additionally, there is a high variability of the patient response to the different types of available treatments, such as the intravitreal injections and the macular laser therapy, which also contribute to these statistics.

The DR could affect to patients with type I or type II diabetes. However, it normally occurs after a few years, manifesting earlier in the case of type I diabetes than in type II diabetes. According to the study carried out by Williams et al. [9], it is estimated that a $2.4 \%$ of the diabetes European population was diagnosed with Diabetic Macular Edema (DME) [9]. Furthermore, in 2009 the clinical study [10] described that half of the diagnosed diabetic patients had never been seen by an ophthalmologist and that DR is the most frequently identified complication, with 250,000 having DR, 25,000 decreased visual acuity and 13,000 total vision loss of at least 1 eye. Every year more than 3,000 diabetics lose their vision irreversibly, $90 \%$ of which could be avoided with regular clinical appointments to control the evolution of the disease. Additionally, a similar study carried out by PREVADIAB [11] in 2012, estimated that a $12.9 \%$ of the Portuguese adult population (20-79 years-old) suffered from diabetes and a 3\% of the patients had DME [12].

In this way, the development of an automatic system capable of detecting and characterizing the three types of ME: SRD, DRT and CME, represents a basic need as a way to, subsequently, determine measurements that could help to predict the patient response to the different types of treatments.

Nowadays, Computer-Aided Diagnostic (CAD) systems are used in ophthalmology as an important tool, due to several advantages such as: the automated detection of pathological eye diseases, the evaluation of the progression of the pathology and the follow-up treatment analysis in combination with different types of eye fundus images. One of the most widely used is the Op- 
tical Coherence Tomography (OCT) image modality, once this technique allows the assessment of macular diseases as well as it enables the analysis of the extent of disorganization in the subretinal layers. Subretinal layers disorganization refers to the variation of the underlying retinal microstructures due to the presence of intraretinal fluid, typically defined as ME.

\subsection{Research Goals and Contributions}

As indicated, the main goal of this dissertation is the development of an automatic system that is capable of detecting and characterizing the three types of ME. Following the clinical classification of reference in the ophthalmological field, MEs are identified as SRD, DRT and CME. For that purpose, OCT images will be used, since it is the most commonly referenced in the literature given the high-resolution of the retinal tissue that this image modality provides.

Considering the retinal fluid accumulation forms swollen regions within the intraretinal tissues, the proposed system firstly delimits and restricts the Region of Interest (ROI). This ROI is constrained to the retinal tissue. Given that each type of edema typically appears in particular regions inside these retinal layer tissues, two-subregions should be identified to facilitate the work: the inner retina and the outer retina. Then, to identify the three types of ME, clinical conditions should be implemented in each region of interest. This process allows the standardization of the identification of the existing ME types, avoiding the subjectivity that is frequently introduced among the ophthalmologists in this analysis. Additionally, given the instantaneous performance of an automatic computational procedure, the work of the specialists is carried out more easily, improving their productivity and, consequently, the positive impact in the quality of life of the patients.

\subsection{Dissertation Outline}

As described as follows, the proposed dissertation is sub-divided into six chapters:

\section{- Chapter 1- Introduction}

The present chapter motivates the importance of the development of this auxiliary computational tool as well as the main contributions to the automatic identification and characterization of the ME presence in the OCT images.

\section{- Chapter 2- Domain Description}

This chapter is structured into four main sections to cover the entire domain description of the dissertation project. The first section allows a contextualization of the eye anatomy and physiology. Then, the following section describes the main ophthalmological techniques used for the visualization and examination of the eye fundus. Subsequently, the section addresses the ME pathology by describing the three main ME types: SRD, DRT and CME. Finally, the last section exposes the recent projects presented in the literature that are also focused on the identification of abnormal intraretinal fluid regions in OCT images. 


\section{- Chapter 3 - Methodology}

Chapter 3 presents the designed methodology for the automatic identification and characterization of the three ME types using OCT images. The proposed strategy is composed in two main stages, each one being described in detail and validated using clinical references. Additionally, this chapter presents the properties of the software solutions required for the development of this project.

\section{- Chapter 4- Results}

This chapter presents the results and validation of the main stages of the methodology.

\section{- Chapter 5- Discussion}

It includes the discussion of the obtained results and the challenges that were faced in the project.

\section{- Chapter 6-Conclusions}

The last chapter presents the final conclusions about the work carried out, the guidelines and the trial results obtained. Finally, some perspectives of future work are indicated in the scope of this theme. 


\section{Chapter 2}

\section{Domain Description}

Macular Edema (ME) is caused by a leak of fluid derived from the blood of the vascularity within the retinal tissue. The presence of this abnormal fluid accumulation leads to severe lesions decreasing the visual impairment acuity and, in more severe cases, it could lead to permanent blindness.

In this way, for a better understanding of the consequences and the disease pathology, the present chapter firstly introduces the human eye anatomy and physiology. Then, considering that this pathology affects to particular retinal tissues, its main structures presented are described as well as their functions. Subsequently, a brief historical contextualization of the medical techniques used for the evaluation of the eye fundus is recorded, where in particular, through the OCT image, it is possible to identify and characterize the ME in three types: SRD, DRT and CME. Next, in the fourth Section, the pathology under study is described in detail (the so-called ME and the subtypes correlated) as well as the main causes and consequences of these retinal diseases. Then, the recent state of the art will be presented. Finally, the chapter ends with a summary of the presented information.

\subsection{The Human Eye Anatomy and Physiology}

One of the most remarkable sensory systems is the vision, where the eyes are the responsible organs for the capture of the images. According to Leonardo da Vinci: "The eye, which is termed the window of the soul, is the chief organ whereby the senso comune can have the most complete and magnificent view of the infinite works of nature" [13]. With this sentence, this artist wanted to depict the importance of the eye, once it is through this organ, that humans capture the major part of the information about the external and surrounding environment. Indeed, the eye organ is a window to the soul, from the point of view that this organ presents particular characteristics to each individual. Both the highly detailed texture of the iris and the fundus blood vessel patterns are unique to every person, providing suitable traits for biometric recognition [14].

For a better understanding of the capture process, the visual system will be described hereafter. This system forms an inverted image of the external world on the sensitive layer of the retina, 
located at the bottom of the eyeball, as illustrated in Figure 2.1. In an operation similar to a camera, the light enters through the cornea, where it is refracted by the retinal tissues. The amount of light that reaches this tissue is regulated by the iris, similar to the aperture in the camera. Finally, the lens focuses the incoming light rays so that a defined image is projected onto the retina. This complex optical system collects the light from the surrounding environment (the rods and cones, photoreceptor cells) as an electrical signal and transmits these signals to the brain through complex neural pathways that connect the eye via the optic nerve to the visual cortex and other areas of the brain.

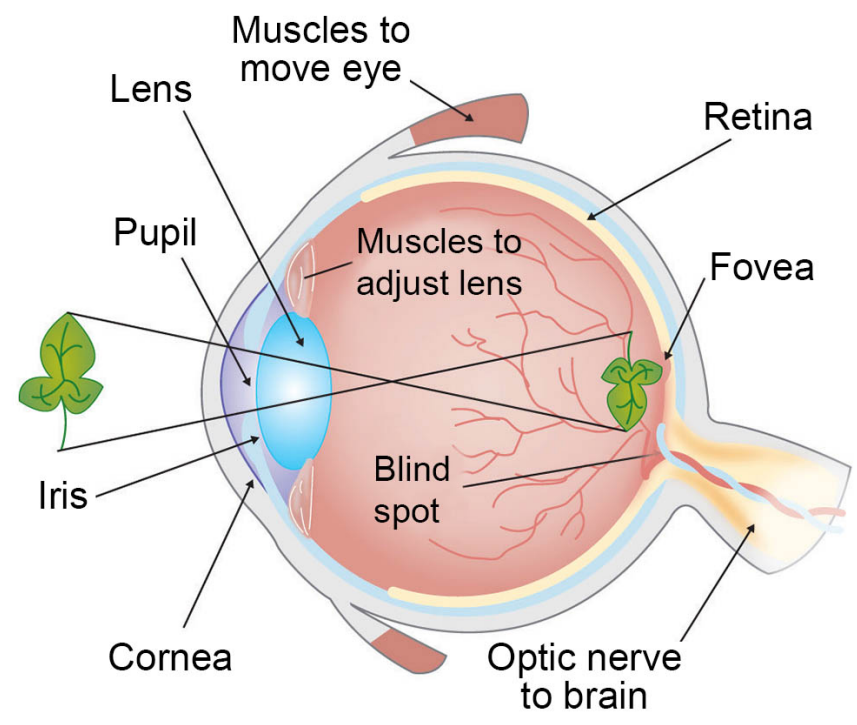

Figure 2.1: Human eye anatomy with the representation of the formation of the captured inverted image [15].

The eyes are a pair of spherical organs located within the orbits cavities. The combination of the function of the two eye globes allow binocular vision granting the perception of the threedimensional image of the surrounding space.

Externally, these structures are protected by the eyelids, eyelashes and tears. Each one of these structures has a vital function in the maintenance of healthy eyes. In particular, the eyelids promote the spread of tears, lubricating the corneal surface, and protecting the eye from dust and foreign bodies, where every single eyelash is embedded into the eyelid by the root. This gives the eyelash hairs additional sensitivity to snap the eye closed in the event of an airborne particle reaching the eye. Finally, the tears lubricate the eye, removing dust and small particles. Figure 2.2 illustrates the external eye anatomy of the eye surface. 


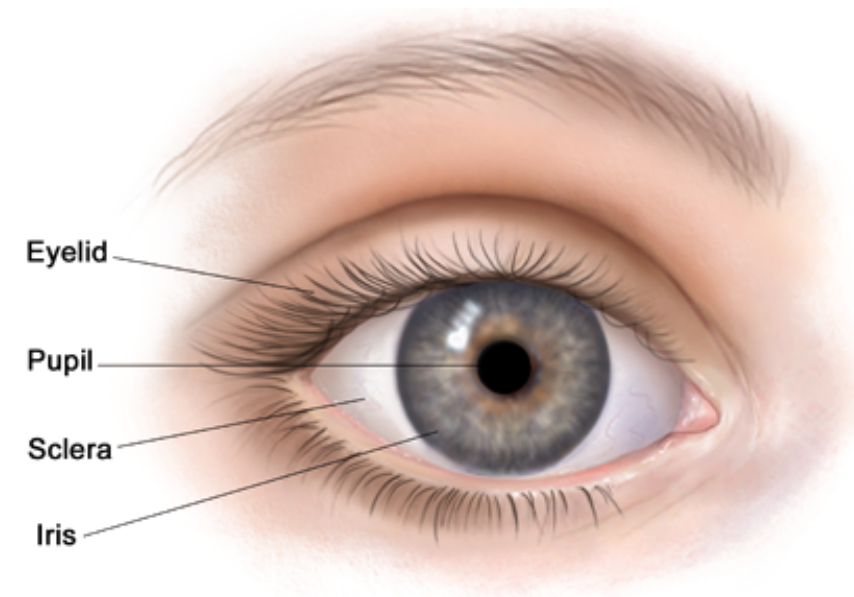

Figure 2.2: External human eye anatomy of the eye surface [16].

The adult eyeball presents a spherical shape with approximately $24 \mathrm{~mm}$ of diameter. The prominent segment with a transparent character occupies a sixth of the globe. It is through this that the light rays enter the organ. In contrast, the posterior segment (of larger size and covering the rest of the eyeball) is completely opaque [17].

The internal wall of the eye globe is composed by three main concentric tunics, as illustrated in Figure 2.3 .

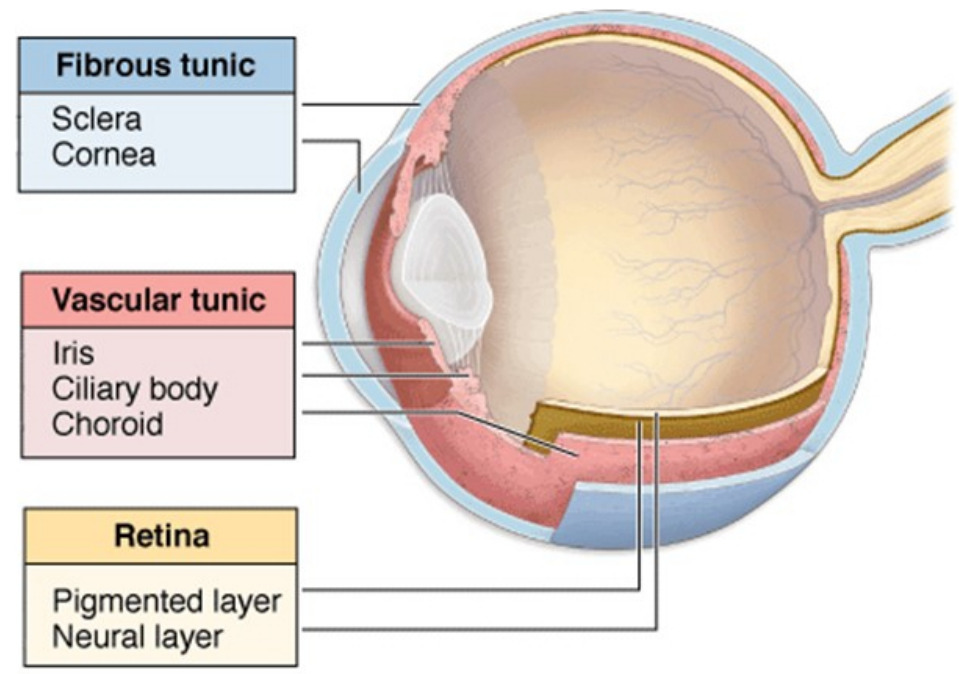

Figure 2.3: The eye is composed of three concentric tunics [18].

- External layer or fibrous tunic. It contains the sclera (the white of the eye) and the cornea (the layer in the front of the eye). The sclera is a fibrous membrane responsible for the maintenance of the eye form, allowing the extra-ocular muscle connection. The cornea is the transparent anterior portion, which allows the light into the eye and participates in the focusing of light onto the back of the eye. 
- Middle layer or vascular tunic. It is called the uvea and it is composed by the choroids, the ciliary body and the iris. The iris is the colored circle surrounding the pupil. It changes the size of the pupil and allows different amounts of light to enter the eye. The ciliary body includes a set of muscles responsible for regulating the convexity of the lens (and with it, the focus). To facilitate the eye movements, this structure secretes an aqueous fluid ensuring the lubrication of the eye. Finally, the choroid is a thin membrane which is irrigated by the blood vessels and it is maintained attached to the ciliary body.

- Inner layer or retina. This is one of the structures that perform the function that gives its purpose to the eye: it absorbs the light and sends the sensory information to the brain to be processed. Near the retina is the posterior pole called macula lutea. This represents the central point of vision. Moreover, just below, it is possible to find the optical disc. This is a blind spot and through which the nerve fibers pass through the retina (we do not see such a blind spot in our vision since the brain itself, with the help of information from the other eye, reconstructs the lost information).

In the following section, the retinal anatomy and physiology are explained in more detail.

\subsubsection{Retinal Anatomy and Physiology}

The retina is one of the most studied parts of the human body given its easy access to any ophthalmic analysis [19].

The retina is the most intern tissue of the eye, being positioned between the choroids and the vitreous body. In the vertebrates, the retina is formed by three layers of cell bodies and two regions of synapses. In particular, the three layers of cell bodies incorporate the rods, cones, bipolar cells

and ganglion cells. The cones and the rods are photoreceptor cells that translate the light into electrical impulses and sends them to the visual cortex of the brain, as illustrated in Figure 2.4 . Moreover, the rods are used in the peripheral vision and they have the particularity of functioning in less intense light than the other type of visual photoreceptors, despite the fact that they do not allow the color detection and they add lower detail information. Furthermore, they are usually concentrated in the outer edges, whereas the cones are densely packed in the fovea. The cones are photoreceptor cells responsible for the color vision and for the vision in relatively bright light, therefore, they are considered as less sensitive to light than the rod cells in the retina. Once the cones response times to stimuli is faster than the rods, the perception of finer details and more expressive changes in images is possible. The cones in the humans allow the trichromatic vision, which is composed by three types of cones namely: S-cones, M-cones and L-cones, with different photopsins [20, 21]. These structures have distinct response curves and though they respond to variation in color it is not equal between them, as shown in Figure 2.5 . 


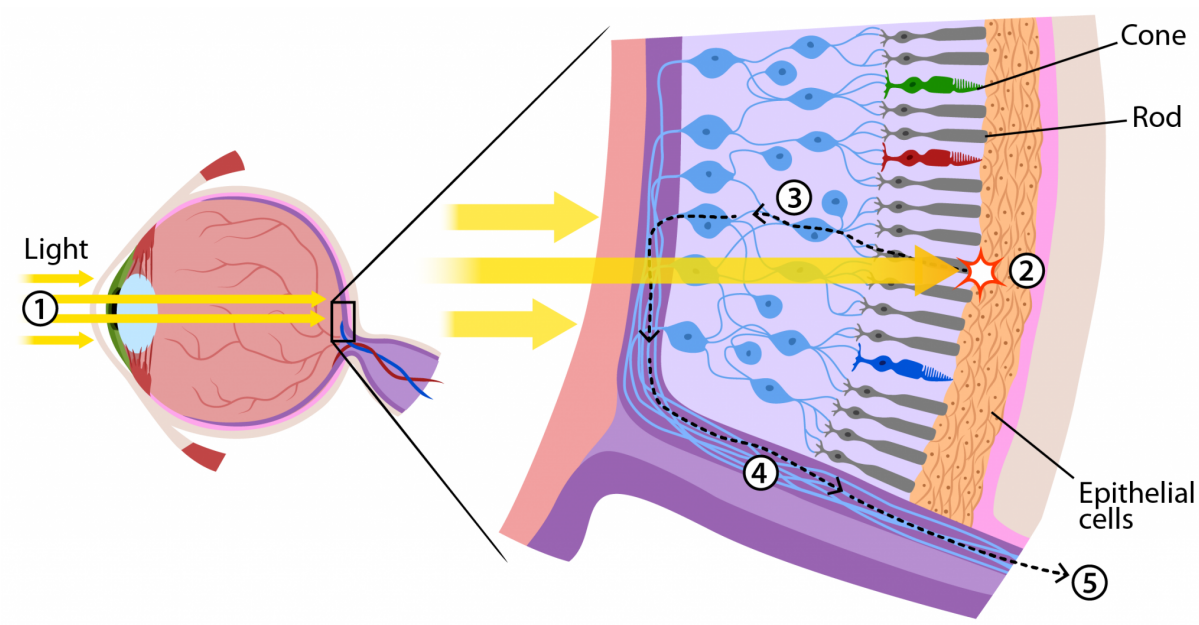

Figure 2.4: Scheme of the path traveled by light through the eye [15]. (1) The light moves through the lens to the retinal tissue. (2) Translation of the light into electrical impulses, by the cones, rods and epithelial cells. (3) The electrical signal passes through a set of neural cells. (4) The stimulus is sent through nerve cells. (5) The optic nerve sends the information to the brain, where it processes the complete image.

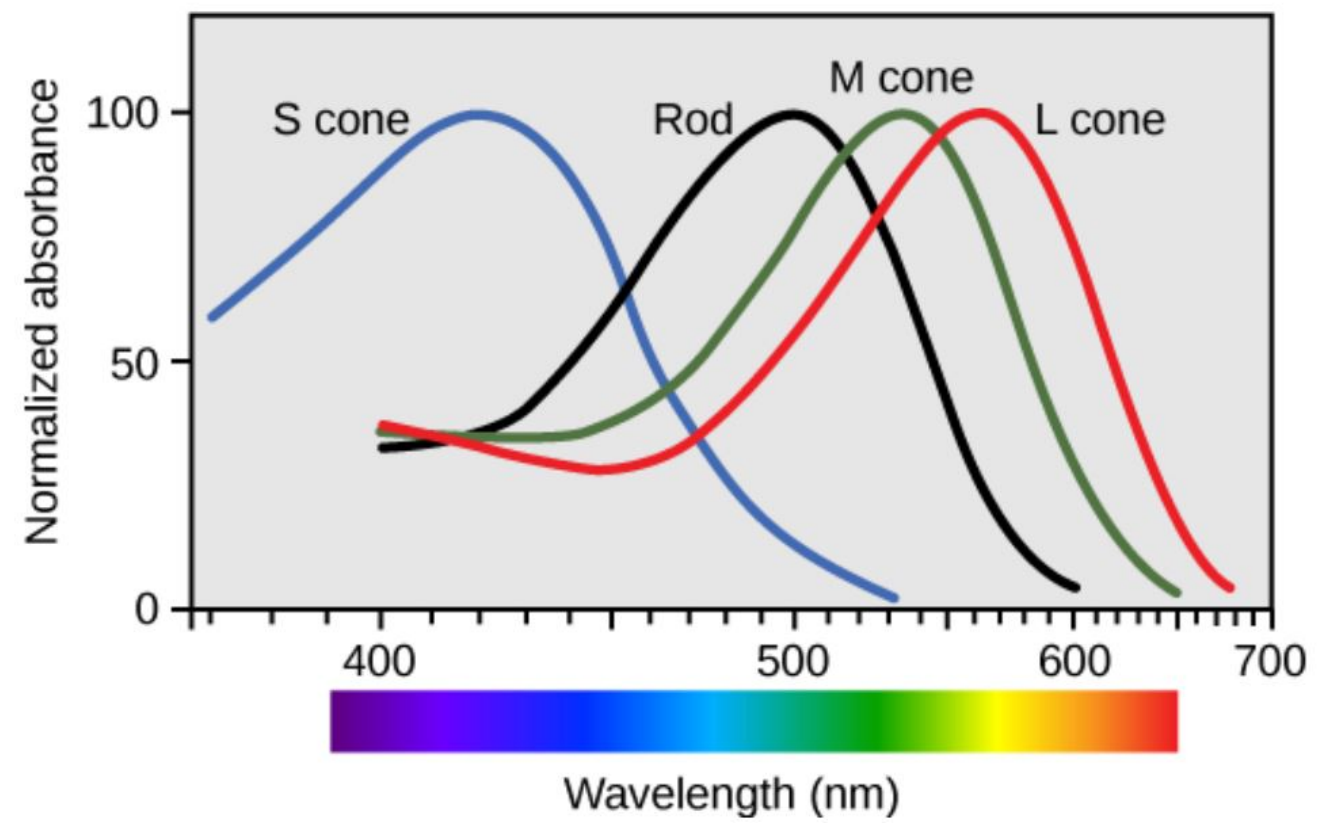

Figure 2.5: Optimal wavelengths for human rod and cone photoreceptors cells [22]. 


\subsubsection{Topographic Organization}

Figure 2.6 represents the principal retinal components, which are hereinafter described [23]:

- Macula. It is an oval yellow spot near the center of the retina of the human eye. This region presents the highest density of cone cells, which are responsible for the color vision [24]. This high density of cones makes the macula the part of the eye where it is possible to see with the greatest clarity and definition. However, the large number of cones results in a lower density of rods.

- Fovea. The center of the fovea is the foveola - about $0.2 \mathrm{~mm}$ in diameter. In the fovea, there is the highest concentration of cone photoreceptors and they are densely packed (in a hexagonal pattern). Despite the high density of cones in the center, the number of cones starts to decrease in the parafoveal region, until becoming almost null, as shown in Figure 2.7.

- Arterio-venular tree. It is responsible for the blood supply to the retina. The retina is irrigated by two blood circulations: the posterior ciliary arteries and the central retinal artery. Once the avascular foveolar region and the periphery of the retina are sufficiently thin to receive irrigation by diffusing the nutrients from the central artery circulation. In opposition, the remaining part of the human retina is too thick, being directly irrigated by the choroidal circulation.

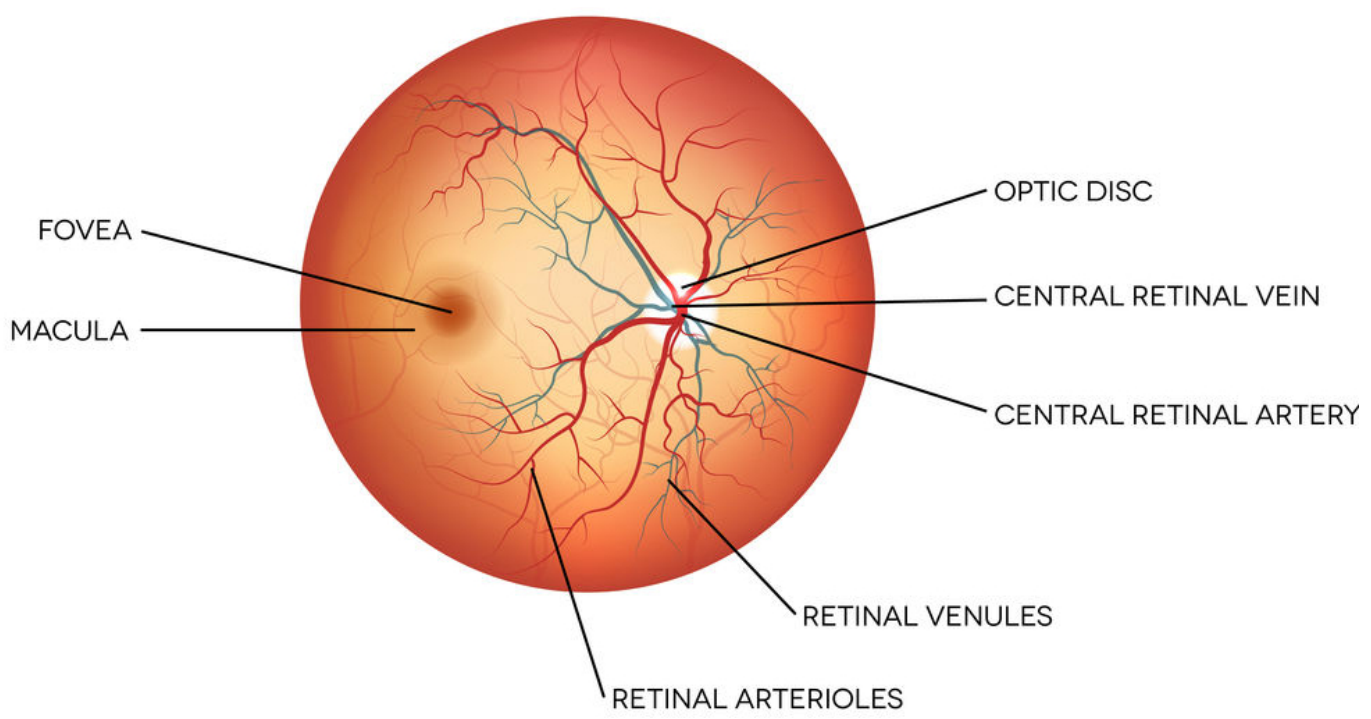

Figure 2.6: Main retina structures [25]. 
(a)

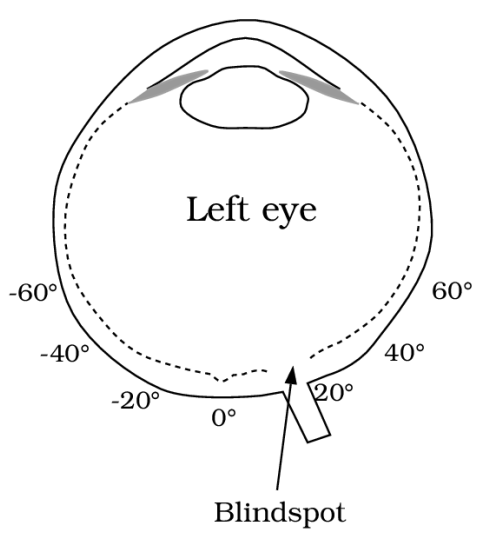

(b)

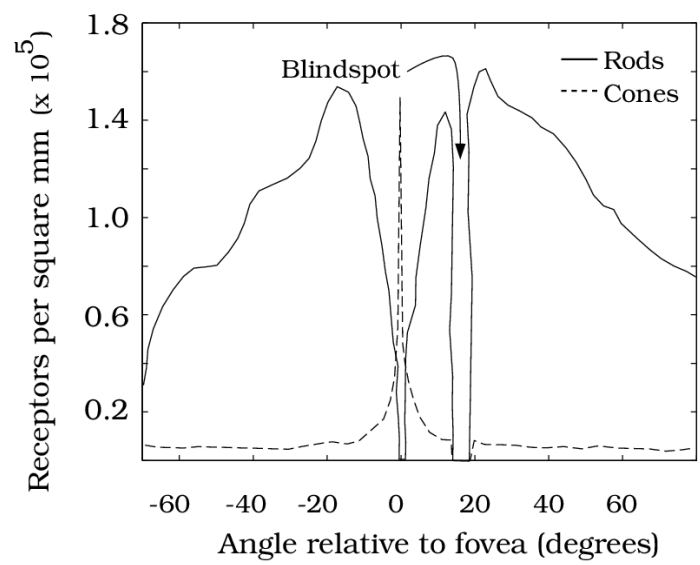

Figure 2.7: Distribution of cones and rods photoreceptors cells across the human retina [26]. (a) The density of the receptors in degrees in the left eye of visual angle relative to the position of the fovea. (b) The photoreceptor cones are concentrated in the fovea, wheres the highest concentration of the rods is in the parafoveal region. No photoreceptors are present in the blindspot.

The ME is characterized as retinal fluid accumulations which form swollen regions within the intraretinal tissues. Therefore, for a better understanding of this pathology, the previous comprehension of the retinal anatomy is vital. Given that, the retinal tissue is composed by ten principal retinal layers, from outside (nearest the blood vessels enriched choroid) to inside (nearest the vitreous humor) [27]. Figure 2.8 illustrates an OCT image with the identification of each one of the ten retinal layers. This technique will be described in detail in the next section.

The ten retinal layers are:

- Inner Limiting Membrane (ILM). It is the thinnest, most transparent and superficial retinal layer, being the boundary between the retina and the vitreous body. The ILM is formed by astrocytes and the end foot of Müller cells.

- Nerve Fiber Layer (NFL). This layer is formed by retinal ganglion cell axons and represents the innermost layer of the fundus. The nerve fiber layer collects the visual impulses from the rods and cones. It is considered the thinnest layer, in the peripheral region, which makes the task complicated for the correct identification. However, near the optical nerve the layer thickness increases.

- Ganglion Cell Layer (GCL). This layer is designed as the innermost nucleated layer of the retina. It is approximately constituted by 1.2 millions of ganglion cell bodies. In particular, the ganglion cells collect information about the visual world from bipolar cells and amacrine cells. Then, the transmembrane receptors transform the chemical messages into the intracellular electrical signal which is integrated within the ganglion-cell dendrites and cell body [28].

- Inner Plexiform Layer (IPL). Also designed as the inner synaptic layer once it consists of synaptic connections between the axons of bipolar cells and dendrites of ganglions cells. 
The IPL contains the synapse between the second-order and the third-order neuron in the visual pathway [29].

- Inner Nuclear Layer (INL). It consists of three different types of cells that are packed closely together. These three types of cells are the bipolar nerve cells, the horizontal cells, and the amacrine cells.

- Outer Plexiform Layer (OPL). In an analogous way to the IPL case, it is a region of synaptic connections; in this case, between the dendrites of the integration cells, they are present in the anterior layer and the axons of the photoreceptor cells. This layer is responsible for dividing the ROI in two sub-regions as the inner and the outer retina.

- Outer Nuclear Layer (ONL). Layer that contains the photoreceptor nucleus cells, being considered as the thickest layer in the foveal area.

- External Limiting Membrane (ELM). It is not a real retinal layer, but a complex conjunctive tissue between the adjacent Müller cells and the internal segments of the photoreceptor cells. It is considered to play a role in maintaining the structure of the retina through mechanical strength [30].

- PL (Photoreceptor Layer). In particular, this layer is composed by two segments as the Inner Segment (IS) and the Outer Segment (OS) forming the ISOS layer or PL layer. This is the only layer that is simultaneously light sensitive and the phototransduction site. All other layers of neuroretina serve the process of transmitting the nerve signals.

- Retinal Pigment Epithelium (RPE). This layer separates the photoreceptor cells from the external retina of the choroid, thus it creates a selective barrier between the external retina and its blood flow.

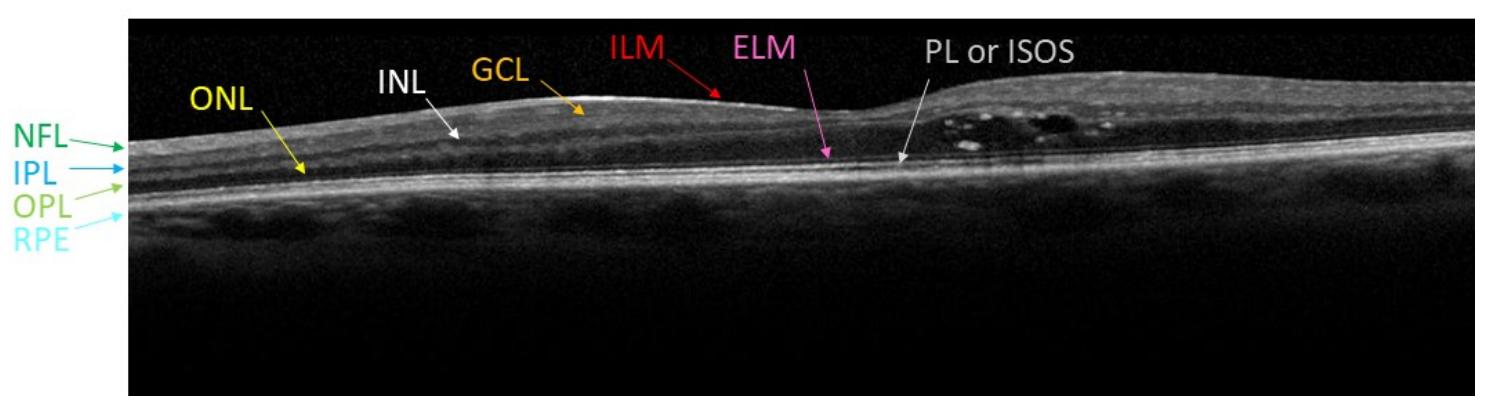

Figure 2.8: Example of an OCT image with the identification of the retinal layers.

Once the layers of the retina are known, it is possible to determine which ones are most important for the proposed project, based on the typical relative positions that each type of ME have within the retina (section 2.4). The ILM and the RPE layers are the two main layers that delimit correctly the ROI as the superior and inferior boundaries, respectively. Another important layer is 
the OPL, given it divides the ROI in two sub-regions: the inner and the outer retina, as previously described. In Figure 2.9, it is represented the division of the retina in two sub-regions as inner and outer retina delimited by the ILM/OPL and OPL/RPE layers, respectively.

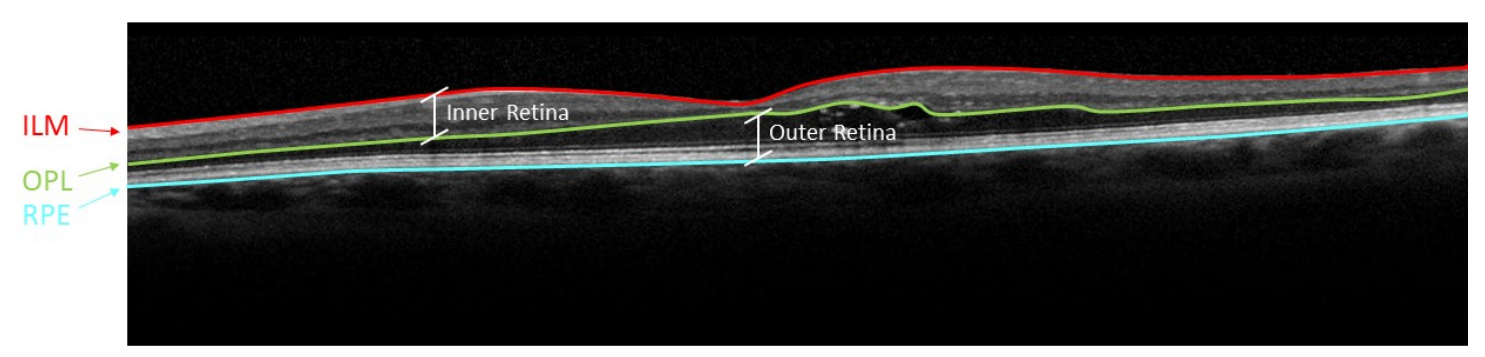

Figure 2.9: Example of OCT image with the inner and outer retina identification.

This retinal segmentation is useful as it facilitates the automatic detection of pathologies that usually present a typical position within the retinal tissue.

\subsection{Image Extraction: Ophthalmoscopy}

As previously described, the retina is the only portion of the central nervous system that is visible from the exterior [31]. Likewise, the fundus is the only location where the vasculature can be directly visualized, without applied indirect techniques to determine the presence of these structures.

The ophthalmoscopy is a medical imaging technique that examines the interior region of the eye, more precisely the back of the eye, in order to get a sense for the patient's overall vasculature. In particular, the fundoscopic exam allows the diagnosis of pathologies, through the detection of modifications in the normal vascular tree. There are several types of ophthalmoscope based essentially on the direct and indirect examination.

\subsubsection{Direct Opthalmoscope}

The direct ophthalmoscope is considered as the oldest and the simplest ophthalmological technique [32]. Commonly, it is a hand-held optical instrument used to inspect the fundus or back of the eye.

The first practical ophthalmoscopes placed the observer's viewing axis and the illumination axis close together by using mirrors or plates of glass. The light is projected through a small aperture where the diameter should be limited to only affect the patient pupil [33]. After, this light is reflected by the patient retina, leaves the patients eye and enters directly into the doctor's own eye, as illustrated in Figure 2.10 .

The modern devices were designed to facilitate the evaluation of the eye fundus by proving a better visualization of the retinal tissue with a magnification of approximately 15 times. This procedure is normally performed in darkened rooms, where the examiner looks for changes in the 


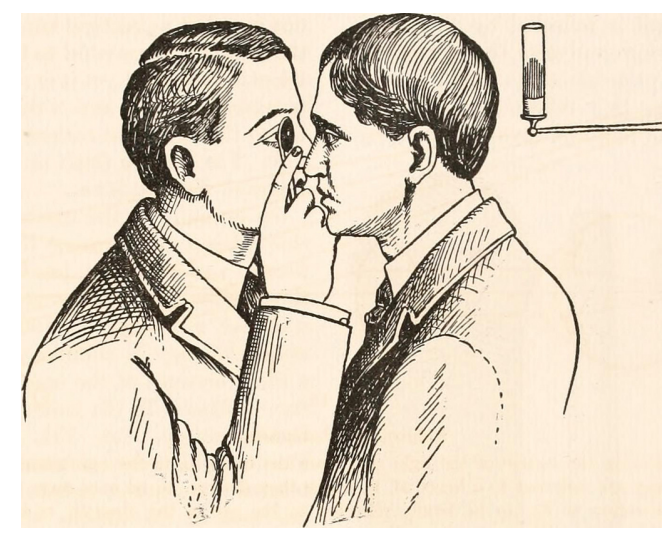

(a)

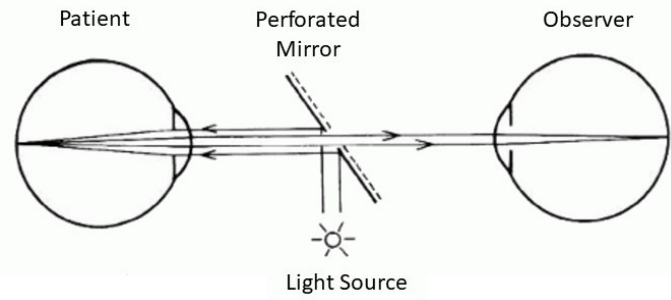

(b)

Figure 2.10: Direct ophthalmoscope technique. (a) Direct ophthalmological examination [34]. (b) Scheme of the direct ophthalmoscope technique [35].

color or pigment of the fundus changes in the caliber and shape of retinal blood vessels and any abnormalities in the macula.

These devices generally contain a handle with a rechargeable battery and a head, frequently detachable, that have a bulb, a set of apertures for the light source, and a set of lenses. The view provided by the ophthalmoscope is monocular, non-stereoscopic (2D), narrow field $\left(5^{\circ}\right)$, as illustrated in Figure 2.11 .

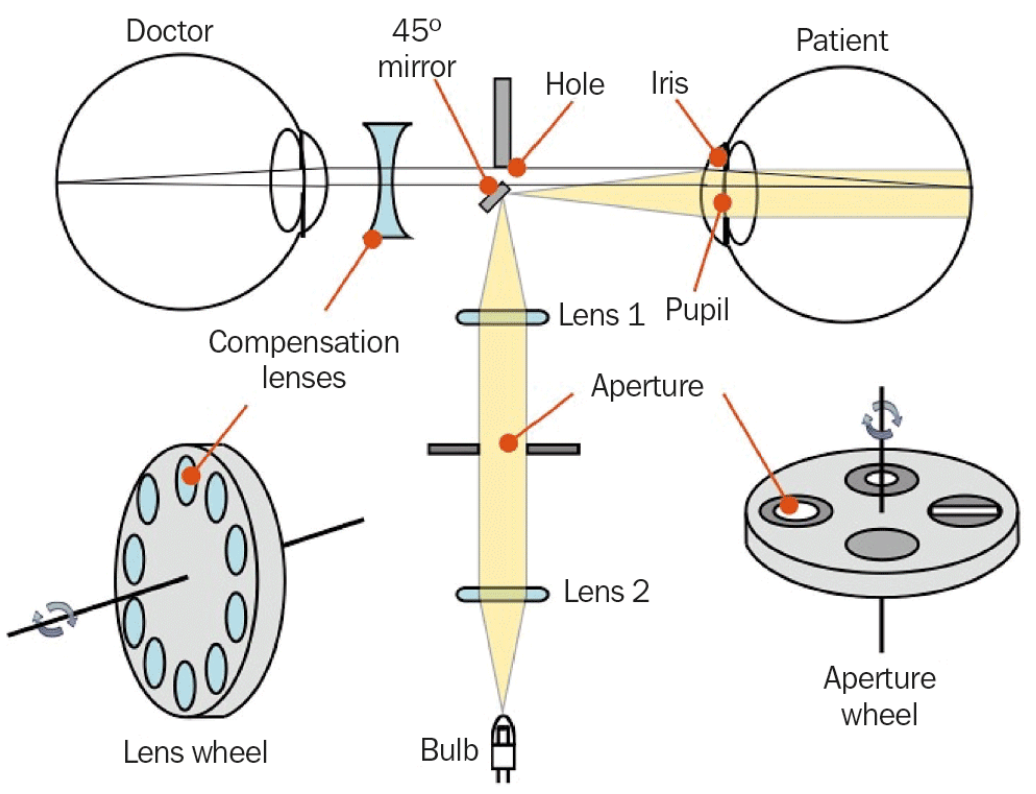

Figure 2.11: Modern direct ophthalmoscope [36]. 


\subsubsection{Indirect Opthalmoscope}

The modern and now ubiquitous approach to the clinical fundus examination is the indirect ophthalmoscopy. This medical examination is called indirect, once the projection of a light source occurs through a condensing lens, which provides the formation of an inverted view of the eye fundus.

This medical examination could be sub-divided into two main categories that include monocular indirect ophthalmoscopes and binocular indirect ophthalmoscopes [37].

The original technique of the inverted ophthalmoscopy was the monocular one, as shown in Figure 2.12. This medical examination offers a high level of magnification and a wider field of view than a traditional ophthalmoscope, but they only offer one view of the interior of the eye. In order to accurately assess a patient's fundus and ocular condition, the ophthalmologist should visualize the fundus of the eye in multiple directions.

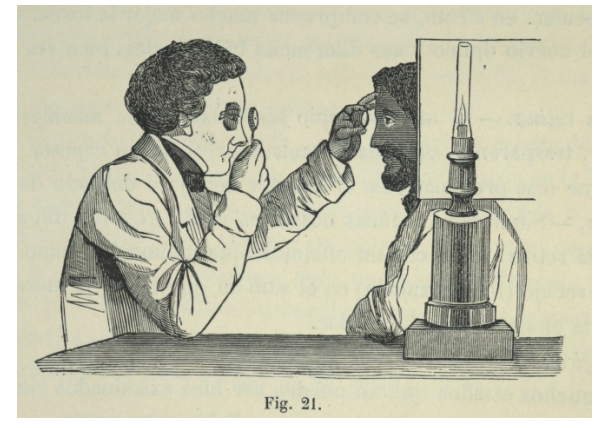

(a)

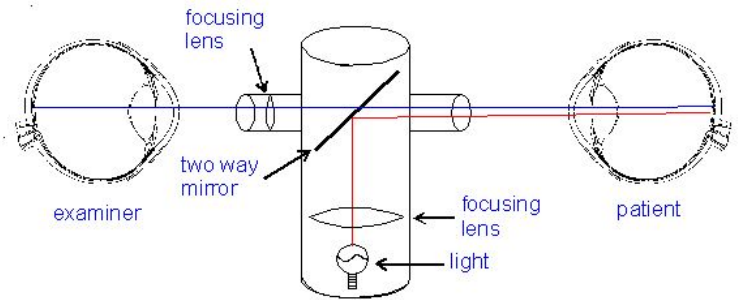

(b)

Figure 2.12: Monocular indirect ophthalmoscope technique. (a) Monocular indirect ophthalmoscope examination [38]. (b) Scheme of the indirect ophthalmoscope technique.

Then, the monocular technique was adapted with the development of the indirect ophthalmoscope, which utilizes binocular vision and thus gives a very useful stereoscopic view of the fundus. This technique projects three elements into the eye, rather than one, allowing the optician to get a three-dimensional rendition of the interior of the eye providing a more thorough examination. Binocular indirect ophthalmoscopes are typically head mounted and some are designed as teaching aids so that lead ophthalmic practitioners can show procedural and diagnostic information to students while conducting the ocular examination. It produces a stereoscopic image with between 2 and 5 times more magnification.

In a dark room, the examiner orientates the head so that light from the internal light source is directed into the patient's eye [39]. A positive-powered condensing lens is held by the examiner at its focal length from the patient's eye, serving two purposes, as illustrated in Figure 2.13

- The lens "condenses" light from the illumination system towards the patient's pupil;

- Light that is reflected from the retina passes back through the lens creating a real, horizontal and lateral inverted image of the fundus situated between the lens and the examiner. 
The viewing system of the instrument (Figure 2.13 (b)) consists of a pair of low-powered convex lenses. This design affords the examiner a stereoscopic view of the virtual image.

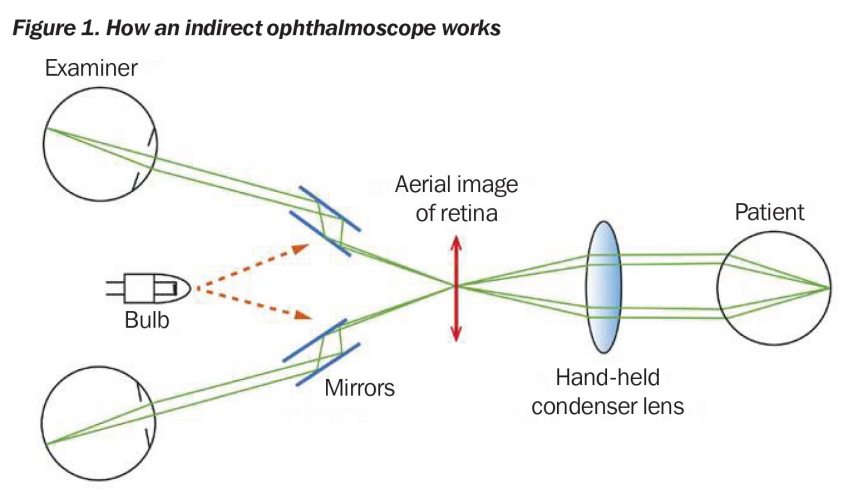

(a)

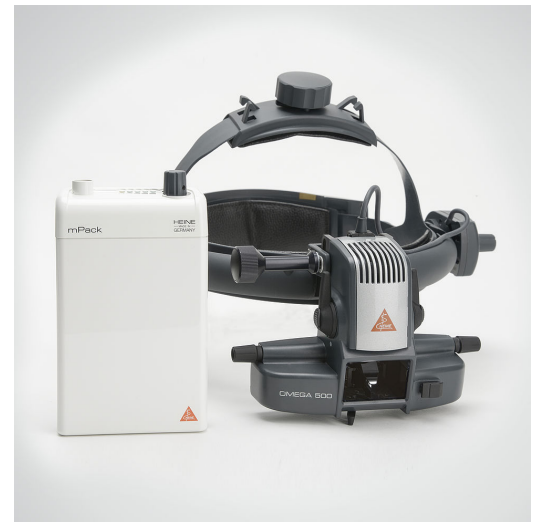

(b)

Figure 2.13: Binocular indirect ophthalmoscope technique. (a) Diagram of operation [39]. (b) Example of a binocular ophthalmoscope device [40].

In sum, the indirect ophthalmoscope offers some advantages over the direct ophthalmoscope, as it permits binocular vision with depth perception (stereoscopic vision), providing a wider field of view. Additionally, it can be combined with scleral indentation to examine the anterior retina, without affecting the refractive state of the patient' eye. Moreover, the accommodation of a larger and brighter light source permits the examiner to penetrate moderate cataracts and to see more retinal detail.

\section{Optical Coherence Tomography}

The Optical Coherence Tomography (OCT) is a modern, and indirect ophthalmoscopy that is commonly used to evaluate the retina. This technique offers more definition and precision of the eye fundus when compared with the simple medical examinations that were previously described.

Since the introduction of the OCT image modality, in 1991, it has become an invaluable tool in the diagnosis and management of different retinal disorders, including ME [41]. It uses lowcoherence interferometry to produce, in real time, a two-dimensional cross-section image of optical back-scattering from internal tissue microstructures, in a way that is analogous to ultrasonic pulse-echo imaging. The OCT is a non-invasive and contactless imaging technique, which has the advantage of evaluating in vivo the histopathology of the retinal tissue, capturing these images with high-resolution [42].

The OCT is based on the Michelson interferometer [43], where the principles are analogous to the technique used for generating an ultrasound image. However, in the OCT technique, light is used instead of sound. The axial resolution is determined by the light source, therefore, it is possible to obtain images of the retina of the eye with at least 100 times higher axial resolution than the confocal microscope, also applied to the eye in vivo. The interferometry does not require 
a direct contact with the tissue in the analysis. However, it is necessary to ensure that the tissue is sufficiently transparent to enable the signal detection.

The Michelson interferometer is constituted by four basic elements as: a laser source, a beam splitter, two mirrors and finally a detector, as shown in Figure 2.14 (a) [44]. The SuperLuminescent Diode (SLD) emits a luminous beam of light, in the infrared spectrum, which reaches the beam splitter. At this point, the light beam is subdivided into two single beams, in the infrared spectrum. One of these light beams hits the retinal tissue whereas the other hits the reference mirror. The light ray that reaches the retinal tissue is reflected as a result of the variations of the refractive index within the tissue (the retina is a heterogeneous medium, as previously indicated). On other hand, the properties of light are known a priori, since the relative position of the reference mirror was previously established. Then, the two reflective beams from the retina and the reference mirror are recombined at the same point in the splitter [45] and redirected to the receiving device. Once the distance of the reference mirror is known, it is possible to determine the distance of the retinal structure that produces the reflectance beam. This information could be graphically combined resulting in an axial image (A-scan). The optical measurement of the axial eye length is obtained by an OCT and it is similar to the obtained image from the ultrasound method imaging, in the A-mode. Using the same device, it is possible to obtain the B-scan, which results from the combination of the multiples A-scans from consecutive regions of the retinal tissue in a single image. The combination of the B-scans could be useful to generate a single three-dimensional image from the retinal fundus resulting in the C-scans, as shown in Figure 2.14(b). Although, for this project, the B-scans will be used.

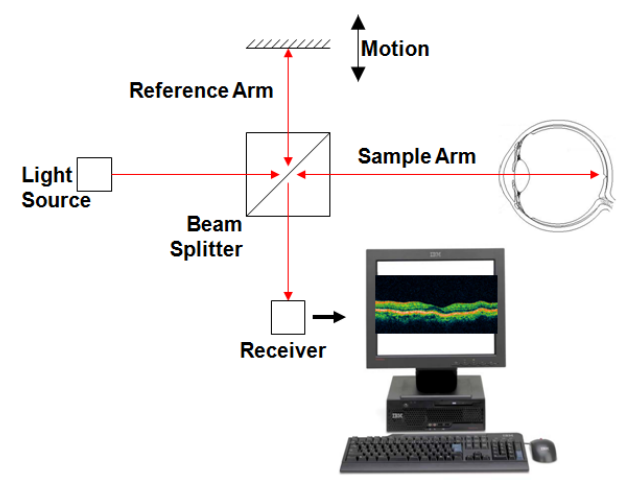

(a)

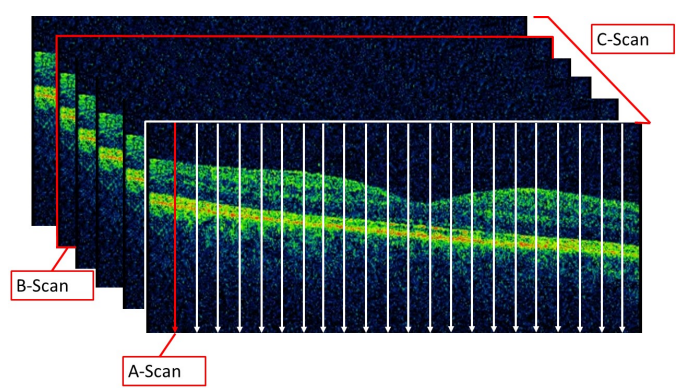

(b)

Figure 2.14: Process to capture the OCT images. (a) Acquisition process of OCT images, based on the Michelson interferometer principle [45]. (b) Terminology for the three types of OCT scans as the A-Scan, B-scan and C-scan.

The OCT technique has been widely used in ophthalmology, given the facility with which light reaches the ocular structures in the anterior and posterior segments of the eye. This exploratory method allows the expert to perform the study on the measurements of the different areas of the retina. The detection of some anomalies are also observed through this methodology. Once the 
diagnosis is made, it continues to be useful for the control and analysis of the pathology evolution by studying the responses of the retinal tissue to the treatments. In Figure 2.15, it is possible to appreciate the use of the OCT capture device with a patient.

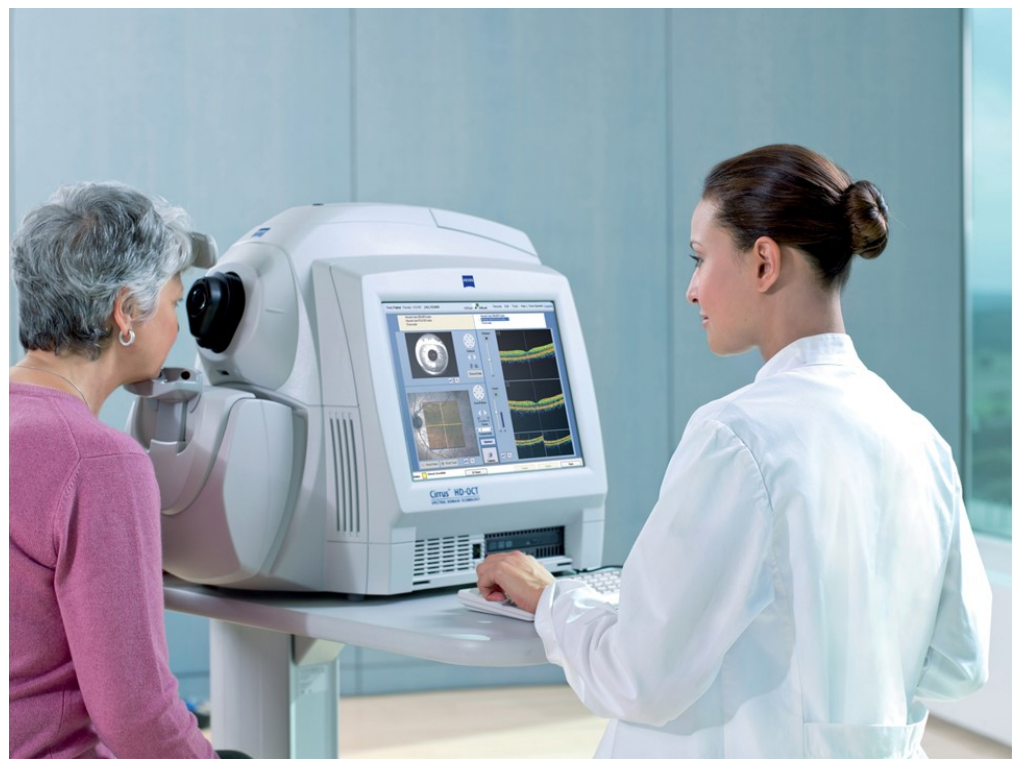

Figure 2.15: Opthalmologic test using the OCT device in a patient [46].

\subsection{Macular Edema (ME) Pathology}

The Macular Edema (ME) is a pathology that decreases significantly the visual acuity of the patients, mainly the ones that suffer from diabetes disease [47]. This pathology is characterized by a nonspecific retinal eye disease with painless visual impairment acuity. If it is not correctly diagnosed or treated it could lead to irreversible blindness. Given that, this section will address the $\mathrm{ME}$ and the causes and consequences associated with this retinal pathology. Following, for each type of ME, the clinical criteria used to recognize their presence through the OCT image modality are described as well as the main treatment options used by the healthcare professionals. At the end of the section, the conclusions of the exposed information are presented.

\subsubsection{Description of the ME Pathology}

There are two main diseases that cause these retinal pathologies as Diabetes and the AMD.

Diabetic Retinopathy (DR) is one of the complications of diabetes and one of the leading causes of blindness in adults due to structural changes in the blood vessels of the retina. As the disease progresses, these vessels become incontinent and release blood fluid into the retinal or the vitreous space causing vision problems. DR can cause vision loss in two ways: (a) Abnormal (neoformed) blood vessels, given they are fragile they easily rupture releasing blood into the vitreous cavity; (b) Blood fluid may exude into the macular region (part of the retina that corresponds to 
the central vision). It can occur at any stage of DR, although it is more likely to occur in advanced stages of the disease [48].

This disease can also occur in diabetic patients treated with oral anti-diabetics (type 2 diabetes) and those taking insulin (type 1 diabetes). It usually appears after a few years, manifesting itself earlier in the case of type 1 diabetes than in type 2 diabetes. The DR is not only dependent on the blood glucose values, but also on other factors such as hypertension, cholesterolemia, smoking habits and another extremely important one that is the genetic factor, namely the hereditary one. In this way, these unhealthy concentrations damage the blood vessels as well as the retinal microcirculation, leading to an abnormal accumulation of fluid within the retinal tissue, forming the ME, as illustrated in Figure 2.16. The ME presence leads to a significant modification of the morphology and as the architecture of the retina, as shown in Figure 2.17. Given that, the retina is a light-sensitive tissue at the back of the eye, and as the macula is the part of the retina responsible for sharp, central and color vision, this fluid accumulation leads to the deterioration of the visual acuity.

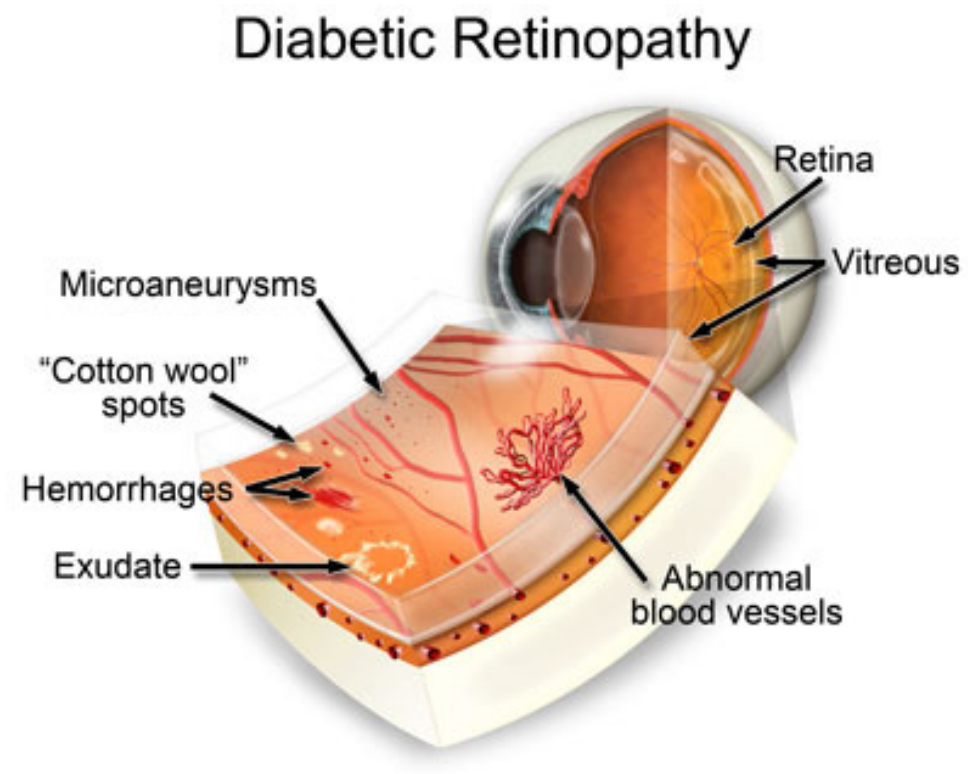

Figure 2.16: Schematic illustration of the biological mechanism forming the ME [49].

\section{Epidemiology}

According to the study carried out by Williams et al. [9], it estimated that $2.4 \%$ of the diabetes European population was diagnosed with DME [9]. Furthermore, in 2009 the clinical study [10] described that half of the diagnosed diabetic patients had never been observed by an ophthalmologist and that DR was the most frequently identified complication, with 250,000 having DR, 25,000 with decreased visual acuity and 13,000 with total vision loss of at least 1 eye. Every year more than 3,000 diabetics lose their vision irreversibly, 90\% of which could be avoided with regular clinical appointments to control the disease evolution. In addition, a similar study carried out by PREVADIAB [11] in 2012 estimated that a $12.9 \%$ of the Portuguese adult population (20-79 

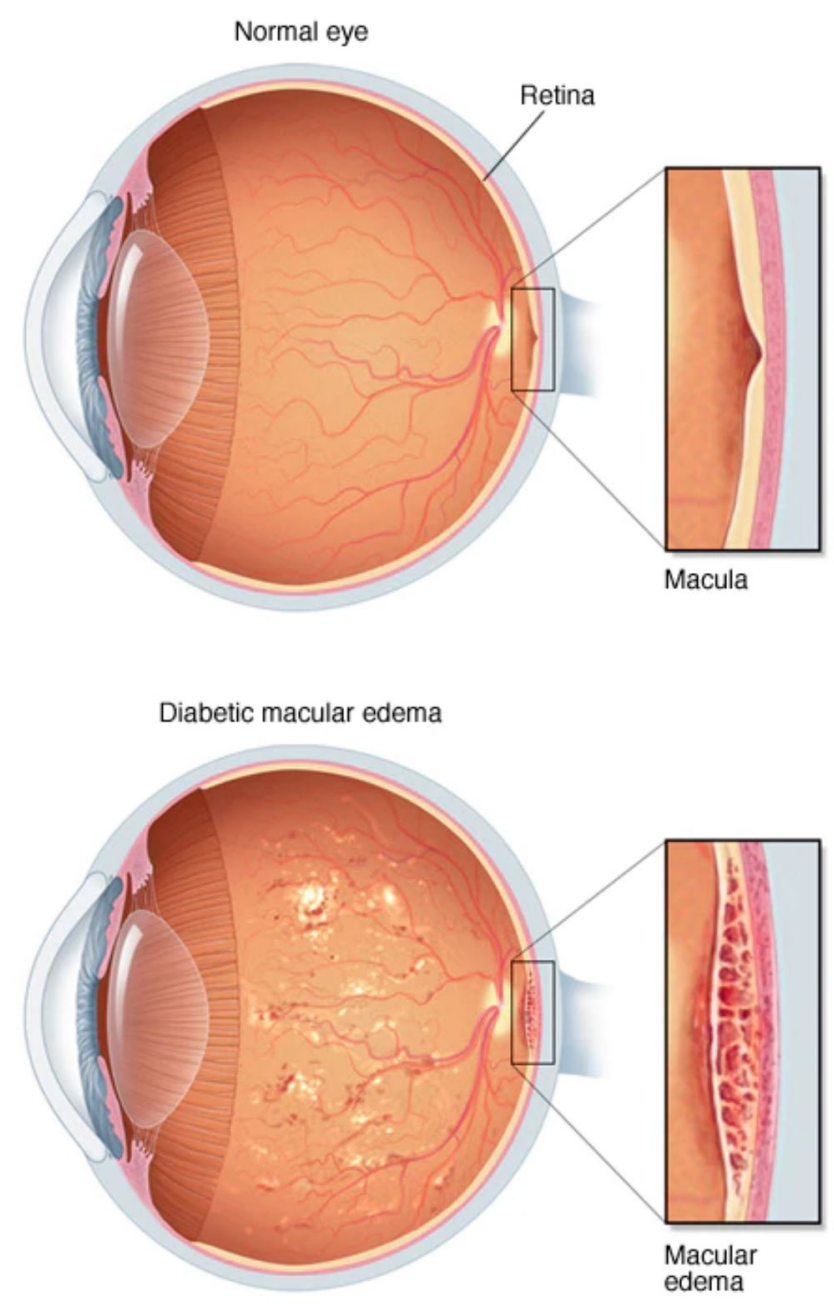

Figure 2.17: Comparative scheme between a normal and pathological eye [1].

years-old) suffered from diabetes and a 3\% of the patients had DME [12]. In 2016, the National Diabetes Program (NDP), together with the Regional Health Administrations (RHA), prepared the first Monitoring and Evaluation Report on Diabetic Retinopathy. From the data analysis, it was found that the number of patients with diabetic retinopathy increased substantially by about $32 \%$ over the previous year [50]. Additionally, in this year the Portuguese Society of Ophthalmology also reported that at least $10 \%$ of Portuguese diabetics had a significant loss, as consequence of the RD, $5.2 \%$ of these patients were blind and about $90 \%$ of these cases could have been avoided if a good monitoring and control had been performed by the national health system [51]. 


\section{Treatment Options}

The prevention of the diabetic retinopathy is critical, given that it is one of the main culprits of avoidable blindness in adults. The treatments are based on controlling blood sugar levels. High blood sugar levels damage the retinal blood vessels, which could lead to the ME formation. However, this monitoring only delays the progression of the pathology by decreasing the chances of vision loss. Depending on the stage of the disease as well as the type of ME, different treatments may be employed where, in some clinical cases more than one treatment could be required [52, 53]. In general, there are three main possible treatments as: pharmacological treatments, laser surgery and vitrectomy, as described next:

- Pharmacological treatments. The "anti-Vascular Endothelial Growth Factor" (VEGF) is one of the most used treatments, which helps to reduce swelling of the macula, slowing down vision loss and perhaps improving the vision. Moreover, steroid medicine is another option to reduce the macular swelling. Both are given as injections in the eye.

- Laser surgery. Laser surgery might be used to help seal off leaking blood vessels, by cauterizing the structures as the blood vessels. This can reduce the swelling of the retina. It can also help to shrink blood vessels and prevent them from growing again.

- Vitrectomy. It is a surgical procedure that removes the retinal fluids from leaking vessels as well as the scar tissue, both removed from the back of the eye.

The goal of DME treatment is to stop the decline and, ideally, to recover vision. Besides the techniques previously described, in recent years new techniques have been included for the treatment of these pathologies as the intravitreal (intraocular) injections of triamcinolone acetonide and the Vascular Endothelial Growth Factor (VEGF) inhibitors, which reduce the vascular permeability and allow the fluid re-absorption. Unlike laser treatment, which potentially causes long-lasting effects, injections require periodic re-treatments. In this case, the cost per injections differs substantially, from approximately $\$ 50$ to $\$ 1,200$ [54].

The causes that lead to the development of the AMD are not well known, although a number of theories have been put forward, including oxidative stress, mitochondrial dysfunction, and inflammatory processes. However, all the theories agree that this disease typically appears in patients with 50 or more years of age. In addition, when this disease is diagnosed, the presence of pigmentary disturbances, drusen (tiny clumps of protein), thickening of Bruch's membrane, and basal laminar deposits [55], generally occur.

There are two main types of AMD as dry (atrophic) AMD and the wet AMD (neovascular or exudative), as described as follows:

- Dry AMD. The dry AMD occurs as a constriction in the macula combined with the merging of tiny clumps of protein called drusen. 
- Wet AMD. The wet AMD is characterized as abnormal blood vessels growing under the retina. This may result in a fast and severe loss of central vision, once the abnormal formation of these new blood vessels tends to break, bleed, and leak fluid, damaging the macula and causing it to lift up and pull away from its base [56].

A more severe vision loss is typically associated with the wet form that occurs in about $15 \%$ of all the patients with AMD but up to $20 \%$ of legal blindness from AMD is due to the dry form [57]. Despite the higher percentage of the population that is affected by the dry form of AMD, there are only treatments for the wet AMD. In the case of the dry AMD, treatments based on antioxidants have been used, once it was clinically tested that they delay the progression of this eye pathology [58]. Recent studies found that a daily intake of certain high-dose vitamins and minerals can slow down the progression of the disease [59].

In the USA, between 2000 and 2010, the number of people with AMD grew a 18\%, from 1.75 million to 2.07 million and in the case of the diabetic retinopathy, the number of cases with this eye pathology increased a $89 \%$, from 4.06 million to 7.69 million [60, 61].

As it is possible to conclude, both retinal pathologies (DR and AMD) have in common the same factor where prevention is the best treatment. An earlier diagnosis of these diseases, as well as the development of more effective treatments, could improve the diagnosis and consequently, the determination of a more adjustable treatment. Moreover, the development of a system that is capable of predicting these retinal pathologies constitutes another strategy as a way of possibly improving the diagnosis and the prevention, consequently increasing the patient's quality of life.

In order to diagnose the diseases at an earlier stage and to evaluate the response of the disease to the treatments, the OCT image modality has been frequently used by ophthalmologists. This technique allows the identification and characterization of the presence of pathological structures, as is the case of the three types of ME. The development of a system capable of identifying these edemas in the OCT images would not only benefit the patients but also the healthcare system, by reducing the costs (in particular a vitrectomy operation can cost about $\$ 3,000$, not counting the risk of the patient suffering side effects after this delicate operation and requiring further care) [62].

\subsection{Macular Edema Types}

The DR as well as the AMD are characterized by the formation of intraretinal fluid accumulations that affect the central vision, once the retinal morphology is modified by the presence of these swollen structures.

Nowadays, ophthalmologists diagnose and characterize the severity of the MEs using the OCT image modality. These scans allow the identification of three different types of fluid accumulations that are classified as: SRD, DRT and CME. This clinical classification was established by Otani et al. [63] in 1999, based on OCT images to identify the fluids accumulations. They used clinical characteristics that are present in the images and classified the intraretinal fluids in three 
types, mainly based on properties as retinal thickness, reflectivity or area of the abnormalities. Subsequently, Panozzo et al. [64] complemented this study also classifying the ME in these types but using 5 parameters to characterize them as: retinal thickness, diffusion, volume, morphology and epiretinal transaction.

Figure 2.18 illustrates the complexity and heterogeneity of this retinal pathology, given the significant variability in terms of morphology, shape or the relative position that each ME type normally presents within the retinal tissue. Typically, the SRD type is characterized by a dome shape at the inferior retinal layers whereas the CME type is defined by the presence of the intraretinal cystoid spaces of low reflectivity separated by highly reflective boundaries that represent the intraretinal cystoid-like cavities [48]. Finally, the DRT type is commonly characterized as a "sponge-like" swelling appearance as a result of a regional fluid spread with reduced intraretinal reflectivity [7, 65].

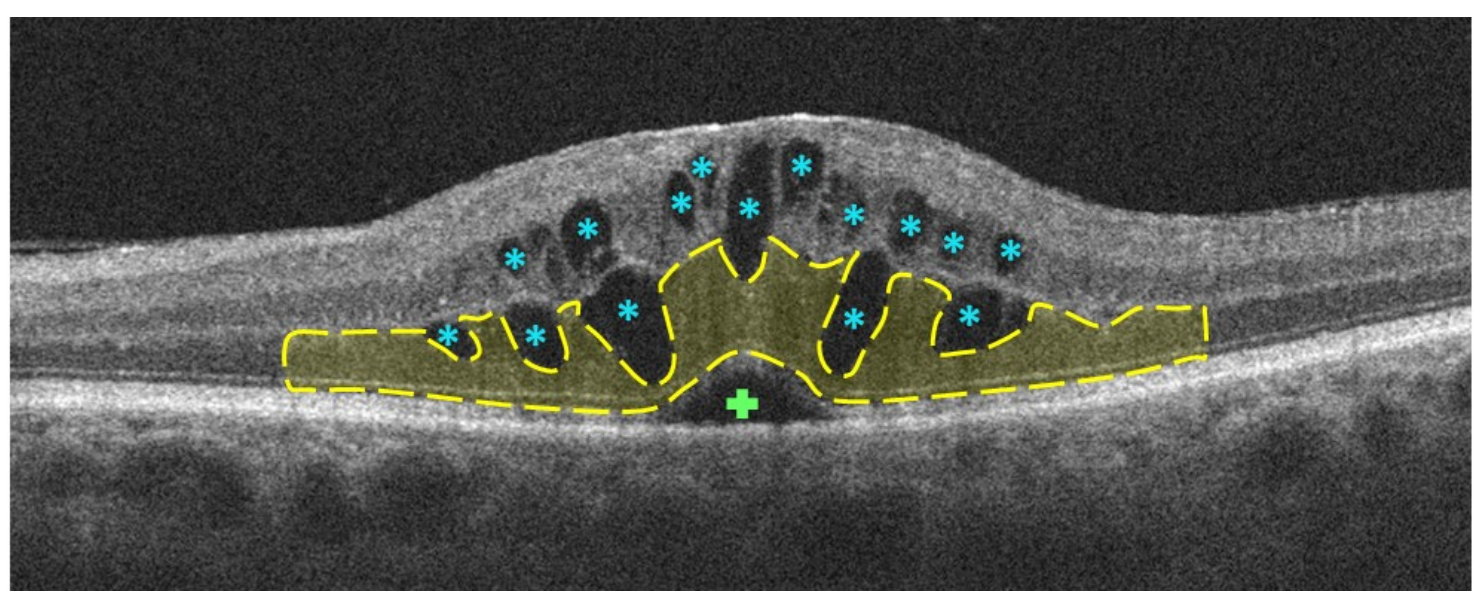

Figure 2.18: Example of OCT image with 3 types of MEs: SRD (+), DRT (- -) and CME (*).

Despite the fact that these three types derive from the same cause, the leak of fluid, their characteristics and visual appearance within the retinal layers are enormously different, as described in detail in the sections: SRD in section 2.4.1. CME in section 2.4.2 and, finally, the CME in section 2.4 .3 .

\subsubsection{Serous Retinal Detachment - SRD}

SRD edemas appear centered in the macula when the fluid is leaked under the ISOS and over the RPE layer regions, within the outer retina, as observed in Figure 2.19. In the OCT images, it can be recognized by the presence of the hyperreflective space presenting a characteristic domelike elevation of a detached retina. The presence of this fluid within the photoreceptor layers leads to a detachment between the photoreceptor layers, creating an abnormal elevation of these layers, which consequently could lead to an increment of the retinal thickness [7]. This edema has the particularity of only appearing one case per each OCT image, in opposition to CMEs, which may occur multiple times. 


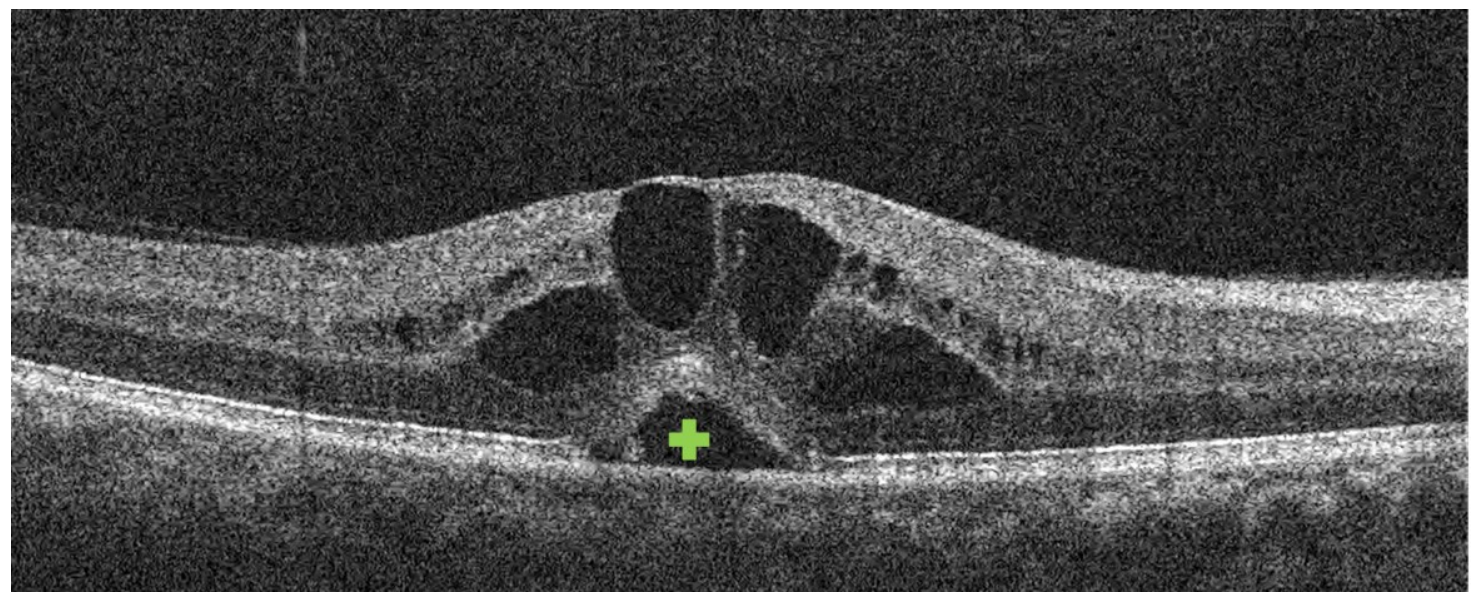

Figure 2.19: Example of OCT image with the presence of SRD edema (+).

\subsubsection{Diffuse Retinal Thickening - DRT}

DRT edemas are typically recognized by ophthalmologists as a retinal swelling of the macula with reduced intraretinal reflectivity. The absence of limiting boundaries creates a particular pattern region commonly designed by ophthalmologists as a "sponge-like" appearance [65], as represented in Figure 2.20. As a result, the thickness of the lower retina region increases, which consequently leads to an increment of the entire normal retinal thickness, modifying significantly the normal morphology and tissue patterns. Moreover, they present a significant variability in terms of patterns and intensities. Hence, in some cases, they could present a brighter sponge pattern whereas in other OCT images they appear as significant dark regions, similar to the background of the image.

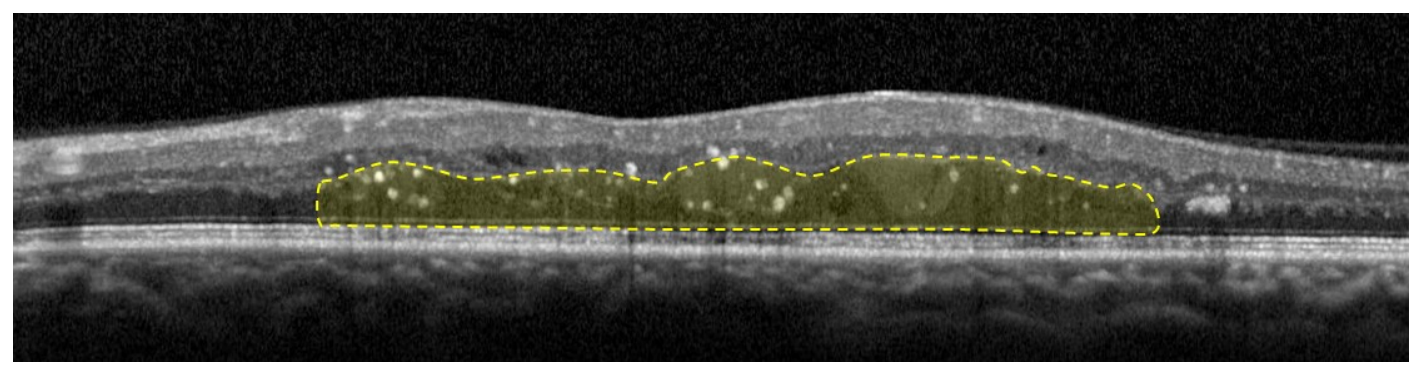

Figure 2.20: Example of OCT image with the presence of DRT edema (- -).

In particular, the identification of these edemas represents an important task, given that they typically appear before CME and SRD edemas, being used by the ophthalmologists as a relevant biomarker for the early diagnosis of the ME disease. 


\subsubsection{Cystoid Macular Edema - CME}

The CMEs are typically characterized as spaces within the retinal tissue, which forms cystoid areas with a low compact intensity profile. These regions are separated by highly reflective boundaries that represent the intraretinal cystoid-like cavities, as illustrated in Figure 2.21. The morphology of these ME could vary from a well-defined round shape to a petaloid-like appearance (like the petals of the flowers). They typically emerge in the inner retina with a significant contrast with the surrounding tissue, as well as a considerable variability in terms of size. In more severe cases, the size of these edemas can be notably increased and, therefore, expand the thickness of the retina, even eventually emerging in the outer retina [48].

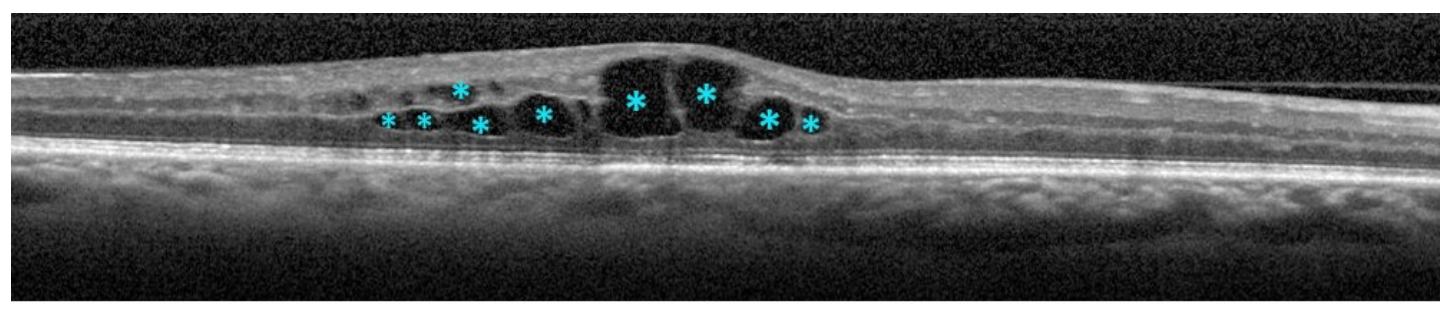

Figure 2.21: Example of OCT image with the presence of CME $(*)$ edemas.

The systems that seek for the detection of these pathological structures are not new in the world of artificial vision and medical imaging. There are several works that have partially addressed the problem of this project. Then, some strategies presented in the state of the art are exposed. However, to date, none scientific proposal studied the automatic detection and identification of the three types of ME diseases using the OCT images.

\subsection{State of the Art}

In recent years, a great development of image processing and analysis techniques in medical applications was achieved, mainly for the diagnosis of diseases. One of the greatest advantages of image analysis lies in its ability to eliminate the subjectivity of the human analysis and the possibility of extracting information that would be difficult or even impossible to obtain otherwise. In addition, the possibility of avoiding extremely tedious and time-consuming tasks for researchers [66] also represents a significant advantage.

This section addresses a review of the state of the art regarding the use and application of image processing and analysis techniques, namely the detection of abnormal fluid regions and the posterior classification in the three different types, using as reference the OCT images.

In recent years, most of the proposed works focused their studies on the automatic detection of the intraretinal fluid. A common step to all these image studies is the image noise reduction, as a way to correctly distinguish between normal volumes and volumes with the ME presence. 
In the work of Sidibe et al. [67], the authors proposed a detection approach for the identification DME. This strategy is based on modeling the appearance of normal OCT images using Gaussian Mixture Models (GMM), detecting as outliers the images with intraretinal fluid.

Lee et al . [68] proposed a deep learning strategy, in particular, a Convolutional Neural Network $(\mathrm{CNN})$, to identify the presence of abnormal fluid accumulation within the retinal tissue.

Roy et at. [69] also implemented a convolutional architecture based on the RelayNet to simultaneously segment the retinal layers as well as the fluid regions that are present in the OCT images.

Alsaih et al. [70] used learning strategies to distinguish between normal volumes and volumes with ME presence. Firstly, the authors proposed a method based on the extraction of Histogram of Oriented Gradients (HOG) features [71] and Local Binary Patterns (LBP) features [72] from four levels using a multiresolution Gaussian image pyramid. Then, a Principal Component Analysis (PCA) and Bag of Words (BoW) representations were applied to reduce the number of dimensions of the concatenated histograms and used in the BoW learning algorithm.

Montuoro et al. [73] proposed an automatic method based on graph theory that simultaneously allows the segmentation of the retinal layers and the existing fluid-filled regions. Firstly the imagebased features are extracted from the raw OCT data and they are used together with manual labels to train a base voxel classifier. The resulting probability map is then used to perform the initial surface segmentation and posteriorly to extract various context-based features. Secondly, these features are used in conjunction with the image-based features to train another classifier. Such context-based feature extraction with the classifier training are iteratively repeated in an autocontext loop with the SRD and CME detection as the final goal.

Given that, following these approaches, none of the announced methods are able to identify and subsequently classify each pathological fluid region in each type of ME in OCT images.

In the next sections, some proposed projects that were specifically developed to detect each type of ME as SRD and CME are described, as well as the simultaneous identification of these edemas. Regarding the DRT case, to date, none scientific work studied the issue of the automatic detection and identification of these ME types.

\subsubsection{SRD Detection}

Many researchers have done clinical studies regarding ME, in particular, in the identification of the SRD edemas, reporting significant findings in macular OCT scans. In the majority of the studies, this ME type is detected as a first stage of the segmentation approaches, as described as follows.

The approach proposed by Sun et al. [74] firstly applied a graph search method to segment abnormal retinal layers. Then, important information is extracted from the DRT region and used in the training stage of the AdaBoost classifier. A total of 62 features are extracted based on shape, intensity and texture characteristics. As a third step, a shape-constrained graph cut method is applied to segment the SRD presence. As the last step, the false candidates are removed by applying adaptive structure elements based morphology methods. 
Hassan et al. [75] employed a Support Vector Machine (SVM) classifier based on five distinct features (three based on the thickness profiles of the sub-retinal layers and two based on cyst fluids within the sub-retinal layers).

Novosel et al. [76] proposed an automatic method to simultaneously segment fluid-associated pathologies and artifacts between the retinal layers. This approach uses the local attenuation coefficient differences of layers and introduces auxiliary interfaces to delineate the fluid region. This strategy allows to determine the abrupt attenuation coefficient variations and topology disrupting anomalies.

In the work of Wu et al. [77], the authors proposed a three-dimensional segmentation method to detect the SRD presence within the retinal tissue. The proposed method involves constructing a probability map from training samples using a random forest classification process. This map results from the linear combination of information from properties like the structural texture intensities and the layer thickness.

In other studies from the same authors, Wu et al. [78] proposed a strategy that firstly segments the intraretinal fluid using a faster R-CNN, then using an effective RPE layer segmentation that they localized on the candidates to SRD edema.

\subsubsection{SRD and CME Detection}

Once the SRDs and the CMEs present a well defined morphological shape and a significant contrast with the surrounding tissue, these edemas are being currently detected simultaneously. In the present literature, the machine learning strategy also dominates this subject.

In the work of Syed et al. [79], they extract 8 distinct features, in particular, 3 based on retinal thickness profile of the right side, 3 based on thickness profile of the left side and 2 based on the top surface and cyst spaces within the retinal layers, in order to train a supervised SVM classifier.

Schleg et al. [80] proposed a method based on a deep learning strategy to automatically detect and quantify the intraretinal cystoid fluid and the subretinal fluid. Given that, a neural network comprising 2 processing components was performed, an encoder that transforms an input image into an abstract representation and a decoder that maps the abstract representation to an image of clinical class labels assigning each pixel to a class such as normal tissue, SRD and CME.

In the work of Lu et al. [81], a method for the automatic segmentation of abnormal fluid regions is proposed, as CME and SRD edemas. In particular, the proposed methodology uses a Fully Convolutional Neural Network (FCN) for the segmentation of these abnormal fluid regions within the retinal tissue.

\subsubsection{CME Detection}

The detection of CMEs is the most common technique in these studies when compared to other studies that focus on the individual detection of the other two types of edemas.

In general, these methods typically begin with an identification of the layers of the retina (since they delimit the region of interest, the area where the cystoid bodies may appear). Most of the 
proposed methodologies are based on a first initial detection of cyst candidates within the layers of the retina, followed by an analysis in terms of shape and/or intensity properties in combination with post-processing stages to reduce the False Positive (FP) rates. In general, the works used methods based on the analysis of the image intensities and the gradient intensities using threshold methods and a flooding process, respectively. Finally, machine learning is also being widely used to identify these structures.

The first works in this field were based on a segmentation of candidate regions by the application of a threshold method (the pixels that are above a gray level are considered from one class and below, from another). In particular, the work of Wilkins et al. [82] used 31 levels of gray (obtained empirically) as values for thresholding the image. This method was gradually complemented with other techniques given the high variability of the samples (dependent on the configuration of the OCT system).

Roychowdhury et al. [83] used it as the main discriminant (also employing an empirical threshold value) and then based on properties such as the maximum pixel value, mean value, perimeter, axle length and other strategies to categorize the cystoid regions.

Gonzalez et al. [84] proposed a work for the automatic detection of the CME edemas by the application of a Watershed algorithm. In order to discard FP, the authors performed a classification based on descriptors of textures, properties of size, shape and position of the different obtained zones.

In the work proposed by Wieclawek et al. [85], they used a method based on the typical low intensity profiles of the cysts, by applying a $H$-Minima Transform. The threshold $H$ has been experimentally adjusted to obtain as result a binary image. Then, correction operations were employed to reduce the false candidate rates, as other structures in the image could have the same intensities. This manipulation relies on clearing border elements and delete those elements that were above the RPE layer.

Taking advantage of the typical contrast of the cysts, characterized as hyperreflective regions with a circle shape, the flooding methods in these regions are being applied. The watershed transform is one of the available approaches, being therefore one of the most used.

Girish et al. [86] employed a prior marking by $K$-Nearest Neighbors (KNN) to find possible candidate regions. Subsequently, they examined the gray level of these regions to discard FP. Finally, they performed a flood of regions that (in theory) should result in the filling of the low intensity areas to obtain the specific morphology of the cysts. Since this technique is vulnerable to FP in areas with similar gray levels of the cysts, they apply a post-processing stage with a list of rules to discard them.

The work of Zhu et at. [87] was designed to detect the presence of CMEs and the Macular Hole (MH) (another problematic in the ophthalmological field). However, focusing on the strategy implemented for the CME identification, they firstly employed an AdaBoost classifier trained with 57 features to generate the initialization results. Secondly, an automated shape-constrained graph cut algorithm was applied to obtain the refined results, finally by removing the false candidates to CMEs. 
One of the least explored fields is the analysis of the texture properties of the image. In the works of Moura et al. [88], each region with the retina was firstly analyzed, in order to identify the presence of the cyst. Then, a total of 189 features were defined. These features included intensity and texture-based properties, once it helps to maximize the discrimination power of the cyst identification with respect to other structures and patterns of the retina. Then, representative classifiers were trained and tested as the Linear Discriminant Analysis (LDA), the KNN and the SVM.

Rashno et al. [89] proposed the application of a neutrosophic transformation and a graphbased shortest path method to segment fluid-associated and cystoid regions.

Deep learning was also recently introduced in the issue mainly for cystoid edemas, Gopinath et al. [90] proposed a method also using a CNN implementation for the segmentation of CMEs followed by a post-processing step using clustering to refine the previously identified cystoid regions.

In the work of Girish et al. [91], the authors proposed a FCN to automatically capture both micro and macro-level features for the characterization of the cystoid structures.

Summarizing, Table 2.1 lists the previous enunciated works, allowing a global analysis and comparison among the different proposals. 


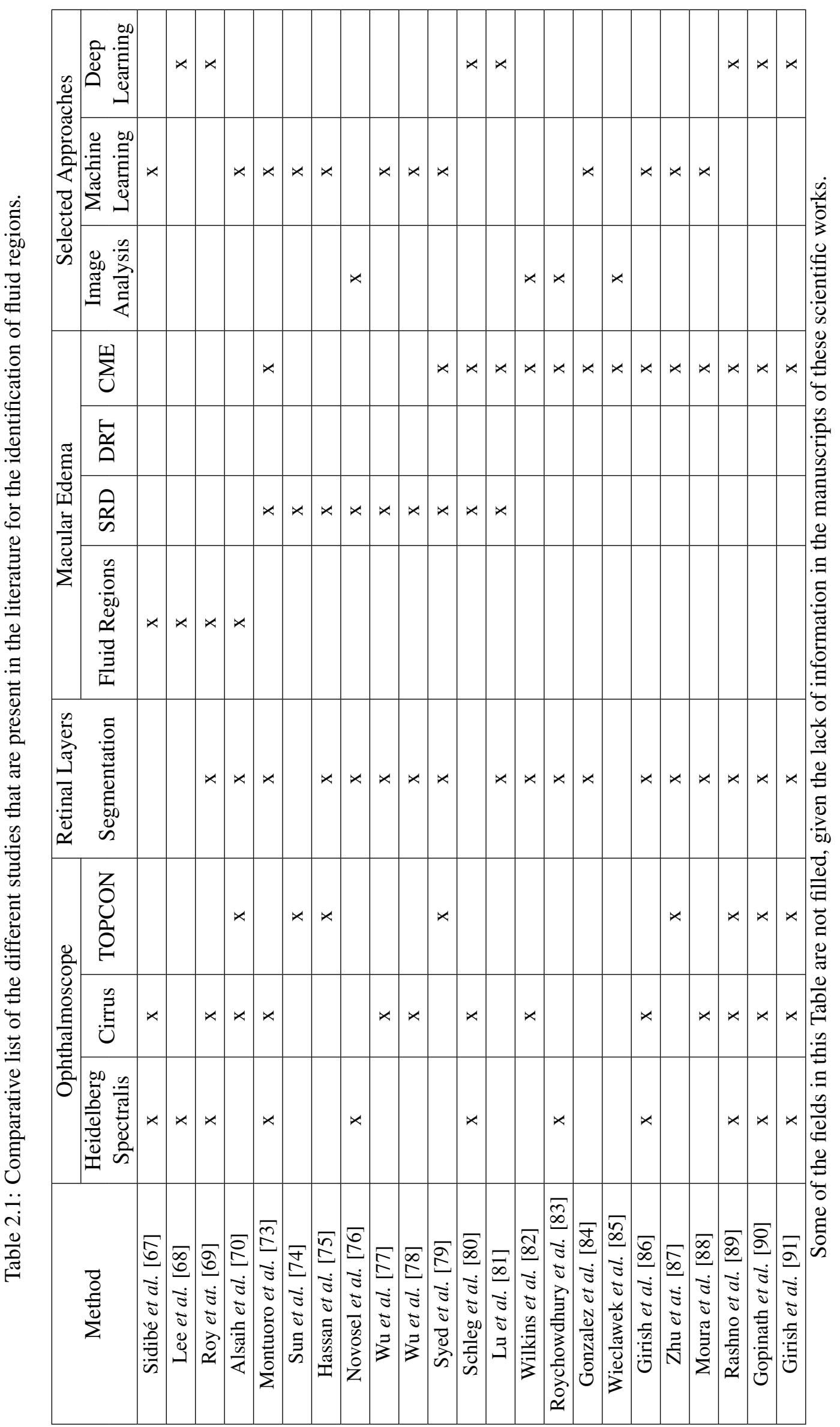




\subsection{Summary}

Following the structure of this chapter, it was possible to acquire the basic knowledge about the eye anatomy and physiology. To analyze and to evaluate the eye fundus, two main medical examinations could be performed using direct or indirect ophthalmoscopes. Given that, the basic principals of each technique were described in detail in this chapter as well as a comparative analysis of the advantages and disadvantages of each technique. In particular, the most used technique is the OCT image modality, as an indirect ophthalmoscope, which allows the detection and classification of the ME edemas in the three main types: SRD, DRT and CME, following the clinical criteria in the field.

Regarding these clinical criteria, several studies applied them in their systems, as a strategy to automatically detect the presence of abnormal fluid regions. In particular, the majority of these works firstly identify the retinal layers to facilitate the ME identification by decreasing the region of search. Then, within this region, different strategies are implemented according to the goal of the study. However, it was possible to conclude that despite the significant importance of the DRT identification (as a relevant biomarker for the diagnosis of the ME disease), to date none scientific proposal studied the issue of its automatic detection and identification of this edema type neither the simultaneous identification of the three ME types. 


\section{Chapter 3}

\section{Methodology}

This chapter describes the designed methodology for the automatic detection and characterization of the three types of ME using the OCT images. For each ME type, we developed a specific strategy that combines medical criteria and image analysis techniques. Additionally, this chapter reports the computational resources required by the designed approaches.

The present project is composed by a multidisciplinary and collaborative research line that involves clinical and technological specialists. Furthermore, this dissertation project is supported by a list of regional and national competitive projects, being under the rights and intellectual property protected and reserved by the involved institutions. The ethical approval was obtained from the Servicio Oftalmología, Hospital de Conxo, in Santiago de Compostela health ethical committee and from the host group - VARPA Research Group, which is affiliated to the Department of Computer Science of the Faculty of Informatics of the University of Coruna (FIC) and to the Research Center of Information and Communication Technologies (CITIC). The medical information, as the OCT images, provided from this clinical institution were renamed by the ophthalmologists before being provided for the system validation to ensure the anonymity of the patients involved in this study.

\subsection{Description of the Main Methodology Stages}

The proposed system receives, as input, the OCT images that are centered in the macula, given that this is the region where the ME types typically appear, offering an optimal visualization of the retinal tissue to identify the hypothetical ME presence.

The proposed method aims to simultaneously identify and localize the three ME types. This strategy allows the characterization of the presence or absence of MEs as well as their amount as useful clinical information for its visual presentation for posterior clinical analysis. The methodology is composed by a set of progressive stages, as illustrated in the diagram of Figure 3.1 . 

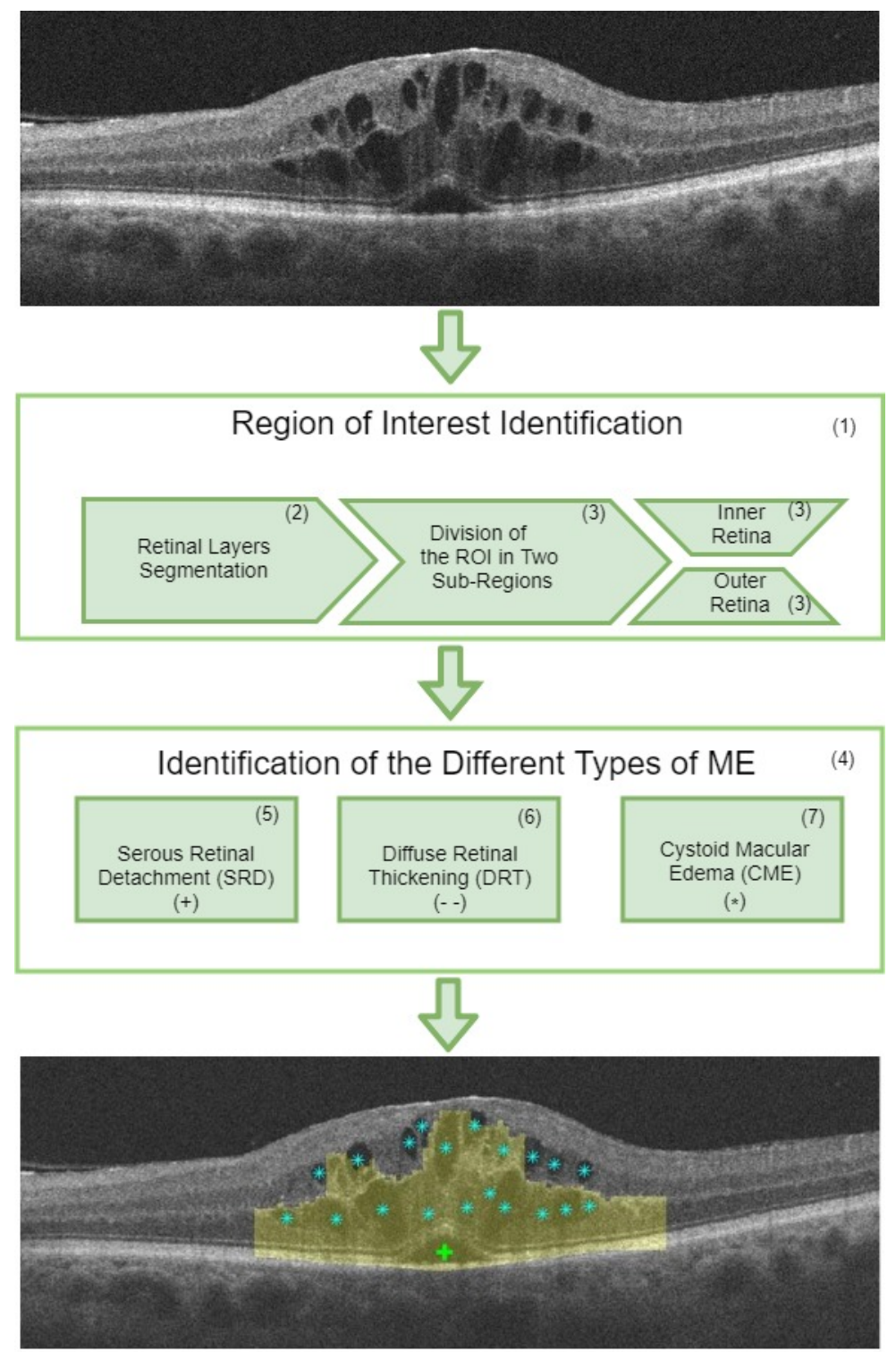

Figure 3.1: Main steps of the proposed methodology for the identification and characterization of the three types of ME as SRD (+), DRT and CME (*).

Figure 3.1 exposes the main organization of the methodology, where we have:

(1) - Identification of the Region of Interest (Section 3.1.1);

(2) - Retinal Layer Segmentation (Section 3.1.2;

(3) - Division of the ROI in Two Sub-Regions: the Inner and the Outer Retinal Regions (Section 3.1.2.1;

(4) - Identification of the Different Types of ME (Section 3.1.3;

(5) - Serous Retina Detachment Detection - SRD (Section 3.1.3.1);

(6) - Diffuse Retinal Thickening Detection - DRT (Section 3.1.3.2;

(7) - Cystoid Macular Edema Detection - CME (Section 3.1.3.3. 
As a first stage, the system detects four main retinal layers as the limiting layers of the analyzed area of interest in the OCT images. This retinal area defines the region of interest (ROI), which is subsequently divided into two main sub-regions: the inner and the outer retina. Then, the system searches each type of ME in three main steps to identify and characterize them individually.

In the SRD and CME cases, multilevel adaptive thresholding approaches were used to detect the presence of these edemas, whereas for the DRT case a learning strategy was implemented. Subsequently, as output, the system provides a labeled image with the identifications and associated types for a better characterization and understanding of the presence of the ME disease.

Despite these three types of ME deriving from the same cause, the leak of fluid, their characteristics and visual appearance within the retinal layers are enormously different, being mainly related to properties as morphology, shape, texture or even the relative position within the retinal tissue. These enormous differences characterize the ME disease as a complex retinal pathology.

Given their characteristics, the SRD and the CME edemas could be directly faced with image processing strategies, basing their identification on the initial selection of possible candidates and a posterior removal of wrong detections using the typical properties that they normally present as shape or relative position, among others, to preserve the real existing ones. For the identification of these edemas, all the defined parameters were designed and determined in collaboration with the clinical specialists, as well as using clinical knowledge from the literature.

Regarding the DRT case, a more complex appearance within the retinal tissue is typically present, with a fuzzier presence and not well-defined boundaries. These characteristics constitute a more complex scenario, motivating the implementation of a learning strategy for their identification, looking for their characteristic texture or clinical properties as the corresponding increment in the retinal thickness, among others, as evidence of their presence.

\subsubsection{Identification of the Region of Interest}

The OCT images contain information about the morphology of the retinal tissue. Given that, when the pathologies emerge within the retinal region, it leads to significant morphology modifications of the tissue. Once each type of ME typically appears in a specific region in the retinal tissue, the proposed system firstly identifies four main retinal layers, to have the region of interest sub-divided in two regions: the inner and the outer retina. This division facilitates the detection of these edemas, once they are typically proliferated in specific relative positions within the retinal tissue.

Next sub-sections, present the designed methodology in detail.

\subsubsection{Retinal Layer Segmentation}

OCT is an optical signal acquisition and processing method that captures the retinal morphology. Similar to other optical tomographic techniques, OCT suffers from speckle noise that reduces the ability of the image interpretation, hardening the differentiation of the surface structures [92]. 
For that reason, the noise reduction is a common preprocessing stage in the analysis of the OCT images.

Next, as shown in Figure 3.2, four main retinal layers were identified to delimit the search space: ILM, OPL, ISOS and RPE. In particular, to delimit the ILM, ISOS and RPE layers we based our approach in the work of Chiu et al. [93]. This approach employs graph theory to represent the OCT images as a graph of nodes. Then, dynamic programming is applied to identify and connect the optimum paths from both sides of the image, which provides the optimal layers. The algorithm calculates the dark-to-light gradient images as they provide information of the limits of adjacent layers, normally being differentiated by the contrast of intensities. Once the gradients are represented by scores, the progressive minimum scored paths are identified to search the main retinal layers. These minimum paths are found by the Dijkstra's algorithm [94]. The original method was developed to detect eight retinal layers, however, in this work, we used this approach to detect three retinal layers as the ILM, RPE and ISOS layers, as shown in Figure 3.3. The first two layers delimit the entire retinal region, as the superior and inferior layers. In the case of the ISOS layer, it was detected as an intermediary step to subsequently identify the OPL layer.

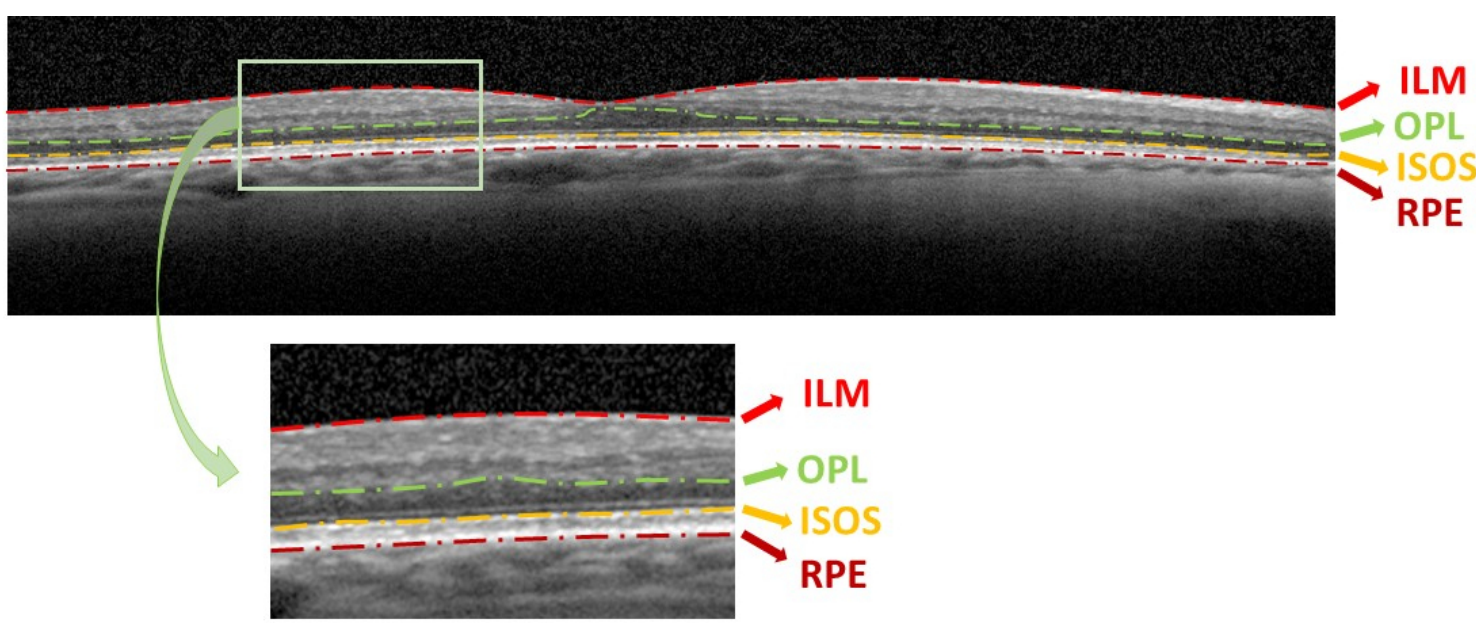

Figure 3.2: Example of OCT image with the identification of the ILM, OPL, ISOS and RPE layers.

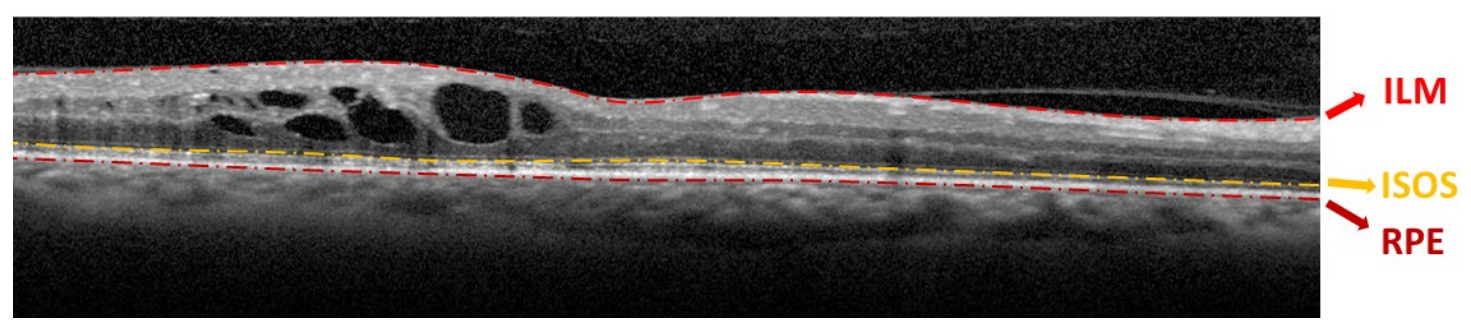

Figure 3.3: Example of OCT image with the representation of three main retinal layers ILM, ISOS and RPE. 
The presence of MEs within the retina leads to a considerable modification and deterioration of the common morphology of the retinal layers. In particular, the OPL layer is significantly altered by the presence of fluid in the outer and inner retina, hardening the correct process of its identification. Thus, the previous method did not present acceptable results in very deteriorated cases. To solve this issue, a new strategy was developed in order to detect this retinal layer, even in more severe pathological conditions, as happens with the presence of ME in advanced stages. Therefore, to solve this issue the previous identification of the ISOS layer is used as baseline to extract the region immediately over it, which corresponds to the OPL layer. Thus, over this layer, $\mathrm{N}$ initial points are randomly generated and used as seed points in a region of growing process [95], as shown in Figure 3.4. The number of generated seeds is proportional to the image OCT dimensions, representing a $10 \%$ of the input image width. In this way, the surrounding pixels in the neighborhood of the seed points are appended with other pixels with similar properties. This strategy allows the delimitation of the entire region over the ISOS layer, which is composed by similar intensity properties. The upper limit of the previous resultant area represents the OPL layer. The use of a significant number of seeds along the image ensures the OPL extraction even in deteriorated pathological conditions, as happens with the presence of MEs in advanced stages.

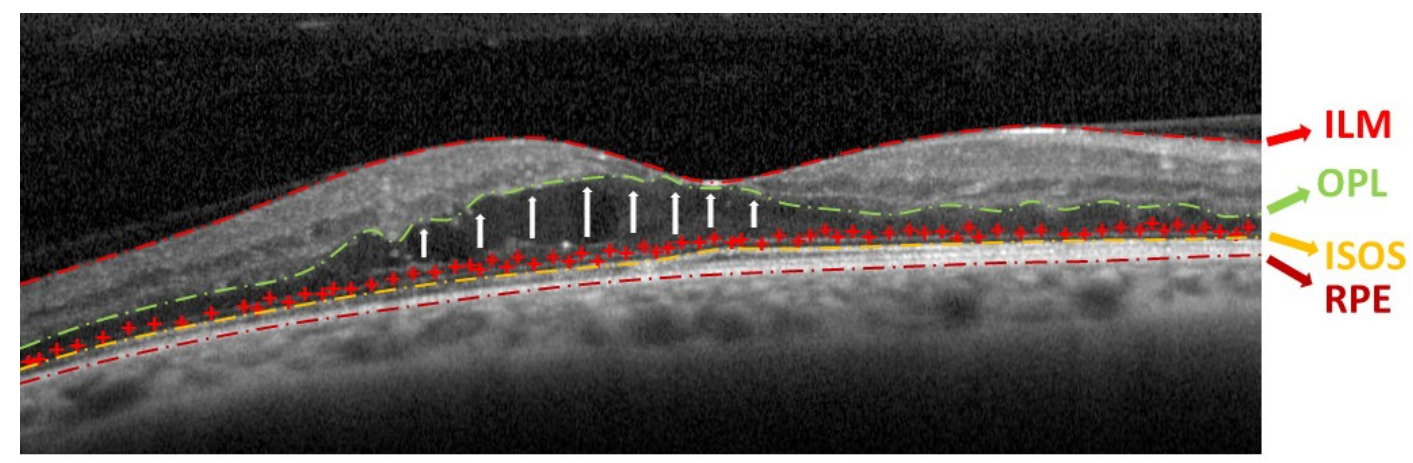

Figure 3.4: Example of OCT image with the identification of the OPL layer by the application of the Region Growing approach.

\subsubsection{Division of the ROI in Two Sub-Regions: Inner and Outer Retina}

The MEs are clinically identified not only by morphology but also by the relative positions where they are typically placed within the retinal tissue. According to the Otani et al. [63] classification, the CMEs normally emerge in the inner retinal layers, defined as the upper region of the analyzed ROI. In opposition, the SRD and DRT edemas typically start manifesting in the outer retina. In more severe pathological stages, the CMEs could proliferate from the inner retina to the outer retina and merge with the DRT edemas [96, 97]. Based on that premises, two sub-regions are identified inside the ROI as the inner and outer retina, using the previously identified layers. This strategy facilitates the detection of each type of ME once the region for detecting each one is smaller, reducing the chances of misclassifications. 
The inner retina can be defined as the upper region of the ROI delimited by the ILM and OPL layers, whereas the outer retina is the lower region which is comprehended between the OPL and RPE layers, as represented in Figure 3.5 .

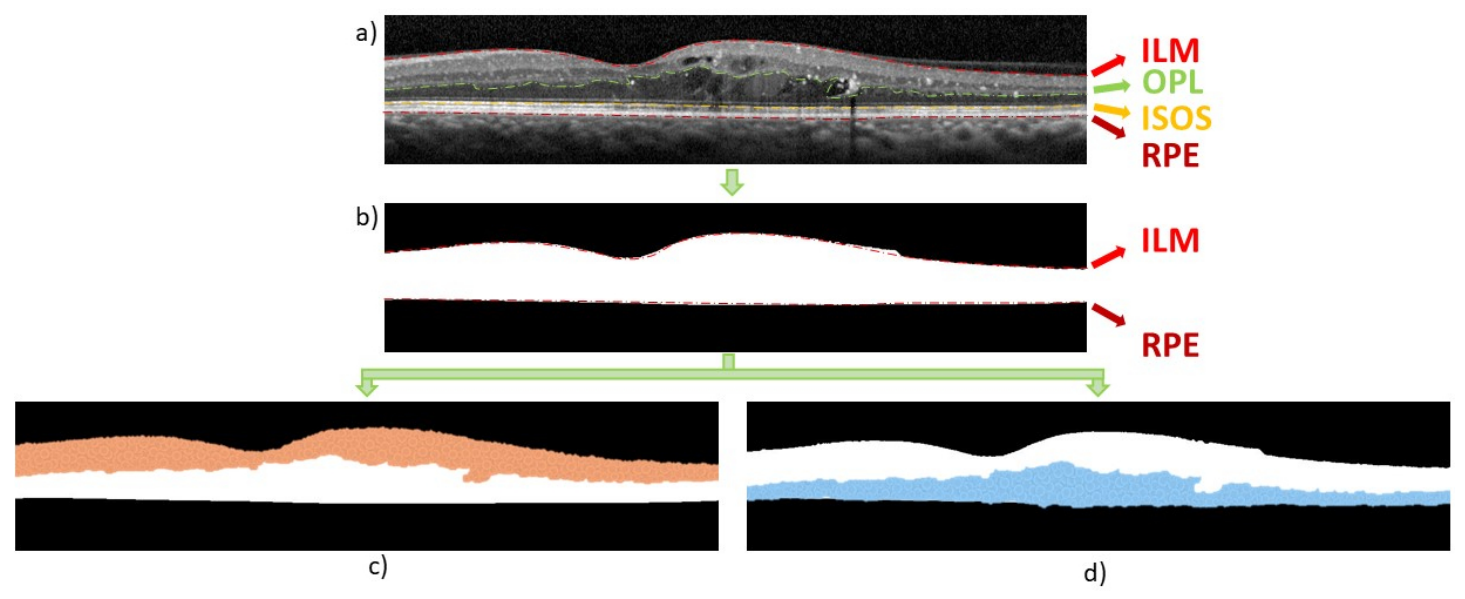

Figure 3.5: Example of OCT image. a) Original OCT image. b) Identification of the ROI mask. c) Identification of the inner retinal region between the ILM/OPL layers. d) Identification of the outer retinal region between the OPL/RPE layers.

\subsubsection{Identification of the Different Types of ME}

Despite the fact that these three types of ME are derived from the same cause, the leak of fluid, their characteristics and appearance within the retinal layers are significantly different. Therefore, the system firstly uses as reference the sub-regions where they typically appear and, then, image analysis approaches are combined with clinical knowledge as individual strategies to identify each type of ME.

\subsubsection{SRD Detection}

SRD edemas appear centered in the macula when the fluid is leaked between the photoreceptor layers. Figure 3.6 illustrates the limiting boundaries where the SRD typically emerge, having as superior and inferior boundaries the ISOS and RPE layers, respectively. The ophthalmologists typically use the OCT images to recognize the presence of these edemas based on two main criteria: relative position and morphology. The first criteria, relative position, is based on the common localization of these edemas as the proximity to the photoreceptor layers and the common centered position over the macula that is characteristic to these edemas. Additionally, these edemas are characterized by the presence of a hyperreflective space with a typical dome-like appearance elevation. However, at early stages, it is complicated to distinguish the typical contrast over the photoreceptor layer and the early proliferation of this ME type, hampering the identification of its presence. 


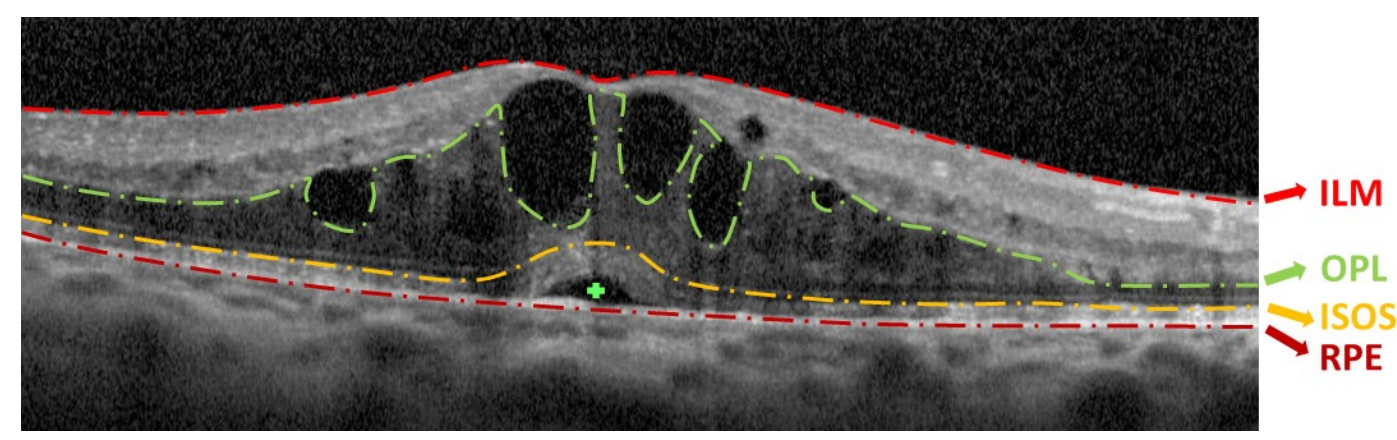

Figure 3.6: Example of OCT image with the identification of the SRD edema (+) in the outer retinal region, more specifically between the ISOS and RPE layers.

Taking this clinical knowledge as reference, the proposed system is developed to automatically detect the presence of these edemas in the OCT images. Given the relative position where they typically appear, the proposed methodology was implemented restricting the search space to the previously identified outer retina. Inside this region, an adaptive thresholding was applied in order to segment areas with identical intensity profiles [98]. The optimal threshold was determined by the value that maximizes the separability between the SRD regions and the normal retinal tissue and also minimizes the separability between the intraretinal fluid accumulation region [99, 100]. The application of this strategy results in several candidates to SRD with low intensity profiles. Next, a list of medical restrictions were implemented in order to reduce the large set of false candidates, described as follows:

\section{- Relative Position:}

SRD edemas are identified by the intraretinal fluid accumulation near the photoreceptor layers, commonly limited by the ISOS and RPE layers, as superior and inferior boundaries, respectively. In this way, to facilitate the search within the outer retina, the candidate should be located near the macular region.

\section{- Minimum Area:}

These edemas present a significant size within the retina, however, we defined that the candidate to SRD edema should present more than two times the area of the Microcystic Macular Edema (MME), which corresponds to an area bigger than 200 pixels $^{2}$ [97, 101].

\section{- Morphology:}

These edemas are recognized by the common dome-like shape. To measure this fusiform shape, we enclosed each candidate in an ellipse form, by considering the minor and major axis of the ellipse of the candidate. For these two parameters, it was considered that the candidate to these edemas should have as minor and major axis a value comprehended between 10 and 250 pixels, respectively, as the usual dimensions that the SRD edemas typically present in the eye fundus. 


\section{- Thickness of the Photoreceptors Layer:}

The presence of fluid in the macular region leads to a decrease of the thickness of the region comprehended between the ISOS and the RPE layers [102]. Using the mean thickness of the ISOS/RPE layers as reference, a window size with a variable height is applied to this region. Then, to be considered as a possible candidate to SRD edema the local mean should be smaller than $95 \%$ of the global ISOS/RPE thickness.

\section{- Intensity Profile:}

As previously indicated, these edemas are characterized as a hyperreflective region in contrast with the surrounded retinal tissue, which is hyperreflective (produced by the photoreceptors layer). Then, to be considered as a possible candidate to SRD edema the local mean intensity should be bigger than 0.17 , value that was determined empirically.

Finally, in OCT image only appears a single SRD edema. Therefore, if two or more candidates fulfill the previous conditions, we only preserve the one that is closer to the foveal center. Once the adaptive threshold process does not produce a precise segmentation of the SRD edema, the center seed of the candidate is used as seed position for a region growing process. This strategy is implemented in order to obtain a more adjusted segmentation of the SRD region. The precise extraction of this intraretinal fluid is useful in the posterior stage of the methodology in the search for the other types of ME.

\subsubsection{DRT Detection}

In opposition to the other two types of edemas previously described, the DRT do not present a well-defined boundary neither a significant contrast with the surrounding neighborhood. In fact, the absence of an enclosing layer enables the fluid to spread off in the outer retinal producing a region with a low intensity profile.

The DRT edemas are defined by the specialists as a swelling of the retinal thickness, with undefined boundaries and a reduced contrast with the surrounding tissue. The presence of this regional leak of fluid within the outer retina produces a pattern typically characterized as a "sponge-like" appearance, as shown in Figure 3.7. However, in some OCT images, these edemas are presented with a bright pattern, whereas in other cases it appears as significantly dark regions, similar to the background of the image. As these edemas occur in the outer retina but above the photoreceptor layer, to search their presence, we remove the photoreceptor region and the SRD edema from the search space, if it was detected in the previous stage. The previous precise extraction of the SRD edema facilitates its removal from the new region of interest. Additionally, the correction of the OPL/RPE region to the OPL/ISOS region also decreases the detection of false candidates with a more precise and restricted region to detect the DRT edema. Given the complexity of this ME type, a learning strategy was applied to this case.

Therefore, each column within the search space is considered using a window of a defined size. In particular, the height of the window varies according to the central column height of the 


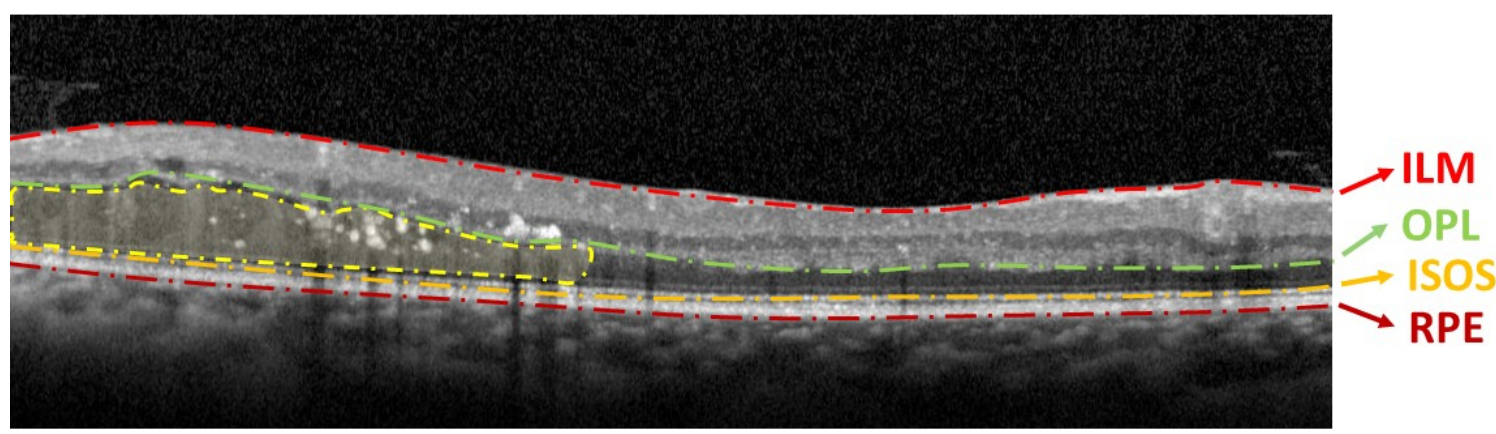

Figure 3.7: Example of OCT image with the presence of DRT edema (- . -) between the OPL/ISOS layers, which belongs to the outer retinal region.

analyzed window, once the thickness of the outer retina is not constant. Given that, the adjustment of the window to the OPL/ISOS region provides a better extraction of the features that efficiently describe the presence of the DRT edemas in the OCT image. Hence, each analyzed window is characterized by a combination of features based on intensity and texture image properties with clinical information, as the retinal thickness, which is normally increased by the presence of the DRT edemas.

In detail, the analyzed features, that are listed in Table 3.1, are the following:

\section{- Global Intensity-Based Features (GIBS):}

Typically the DRT edema is characterized as a region which implies the inclusion of a higher irregularity and a drop of the intensity profiles when compared with the normal tissue of the retinal layers. For that reason, the strategy is based on the extraction of 6 global statistics with the purpose of capturing these alterations. In particular, we have: maximum, minimum, mean, median, standard-derivation and variance.

\section{- Gray-Level Intensity Histogram (GLIH):}

As previously indicated, the DRT edema presents a typical low-intensity profile. Therefore, the intensity alterations also produce a variation in the histogram, with the following measurements being calculated: obliquity, kurtosis, energy and entropy.

\section{- Mask Thickness Analysis:}

The DRT edema is a ME type defined as an intraretinal fluid accumulation that leads to an increment of the outer retinal region but also to the entire retinal thickness. In this way, we considered this clinical criterion and calculated the ROI thickness (ILM/RPE region), the thickness of the outer retina (OPL/RPE region) and the ratio between them. Therefore, the evaluation of the thickness of these retinal regions is an important medical parameter for the determination of the columns that compose the DRT edema.

- Gray-Level Co-Occurrence Matrix (GLCM): 
These edemas are characterized by the typical sponge-like appearance that they usually present. This criterion is commonly used by the specialists to determine the presence of these edemas in the OCT images. For that reason, we capture the pattern of the normal retinal tissue as well as the irregularities of the DRT presence using a statistic method as the GLCM features. In particular, GLCM features characterizes the texture of an image by calculating how often pairs of pixels with specific values and in a specified spatial relationship occur in an image. Therefore, this method measures the simultaneous occurrence of gray level $i$ and $j$ in pairs of pixels $(i, j)$ of the OCT image under analysis, I, which is separated by a displacement vector $\delta=(\Delta x, \Delta y)$ into a 2D matrix, as represented in following Eq. 3.1 .

$$
C_{\delta}(i, j)=\mid p_{1}, p 2: I\left(p_{1}\right)=i ; I\left(p_{2}\right)=j ; p_{2}=p_{1} \pm \delta
$$

Based on the proposal of Haralick et al. [103], different texture features can be extracted from the co-occurrence matrices as: contrast, correlation, energy and homogeneity.

Table 3.1: List of the defined 18 features to identify the DRT presence.

\begin{tabular}{|l|c|l|}
\hline Category & Number of Features & Features Specifications \\
\hline $\begin{array}{l}\text { Global Intensity-Based Feature } \\
(\text { GIBS })\end{array}$ & {$[1-6]$} & $\begin{array}{l}\text { Maximum, Minimum, Mean, } \\
\text { Median, Variance and Standard } \\
\text { Derivation }\end{array}$ \\
\hline $\begin{array}{l}\text { Gray-Level Intensity Histogram } \\
\text { (GLIH) }\end{array}$ & {$[16-20]$} & $\begin{array}{l}\text { Obliquity, Kurtosis, Energy and } \\
\text { Entropy }\end{array}$ \\
\hline Mask Thickness Analysis & {$[11-14]$} & $\begin{array}{l}\text { Thickness of OPL/RPE region, } \\
\text { Thickness of ILM/RPE region } \\
\text { and } \\
\text { The ratio between the thickness of } \\
\text { OPL/RPE and ILM/RPE layers }\end{array}$ \\
\hline $\begin{array}{l}\text { Gray-level Co-Ocurrence Matrix } \\
\text { (GLCM })\end{array}$ & {$[15-18]$} & $\begin{array}{l}\text { Contrast, Energy, Correction and } \\
\text { Homogeneity }\end{array}$ \\
\hline
\end{tabular}

The described set of 18 features were subsequently analyzed using three different feature selectors: Sequence Forward Selector (SFS) [104], Robust Feature Selection (RFS) [105] and SVMForward Selector (SVM-FS) [106]. These feature selectors were successfully applied in similar medical imaging studies [107], to simultaneously reduce the array dimensionality using different strategies but preserving those features with highest discriminative power. In particular, SFS adds each feature to the subset in an incremental importance order, whereas RFS identifies the features using an emphasizing joint $\ell_{2,1}$ norm regularization selecting those with joint sparsity. Finally, SVM-FS uses a linear $\ell 1$-norm SVM for the variable selection, but a non-linear $\ell_{1}$-norm SVM is used for predicting the best subset.

Using the selected features, two classifiers were used to test the potential of the implemented approach: the Naive Bayes and the SVM classifiers. 
In the case of the Naive Bayes classifier [107], it is based on Bayes' Theorem with an assumption of independence among predictors. In simple terms, this classifier assumes that the presence of a particular feature in a class is unrelated to the presence of any other feature. In this way, Bayes theorem provides the calculation of a posterior probability by knowing and determining the likelihood, class prior probability and the evidence, as represented in Eq. 3.2

$$
\text { posterior }=\frac{\text { prior } \times \text { likelihood }}{\text { evidence }}
$$

Which is equivalent to the following Eq. 3.3

$$
P(C \mid X)=\frac{P(X \mid C) \times P(C)}{P(X)}
$$

Where $\mathrm{P}(\mathrm{C} \mid \mathrm{X})$ is the posterior probability, $\mathrm{P}(\mathrm{C})$ is the prior probability of class, $\mathrm{P}(\mathrm{X})$ is the prior probability of the predictor and $\mathrm{P}(\mathrm{X} \mid \mathrm{C})$ is the likelihood which is the probability of predictor given class.

The SVM classifier [108] is a non-probabilistic method that constructs a hyperplane of a set of hyperplanes in a high or infinite dimensional space by separating data samples. Given labeled training data (supervised learning), the algorithm outputs an optimal hyperplane which categorizes new examples. Then, the operation of the SVM algorithm is based on finding the hyperplane that gives the largest minimum distance to the training examples. Therefore, the optimal separating hyperplane maximizes the margin of the training data, where the samples on the margins are called the support vectors, as illustrated in Figure 3.8. Generally, the SVMs converge to linearly separable data. However, in some cases, the data does not present a linear separability, the hard margin can be relaxed allowing some misclassification.

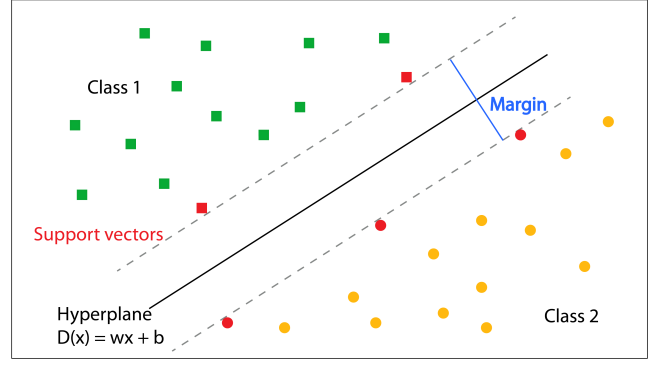

(a)

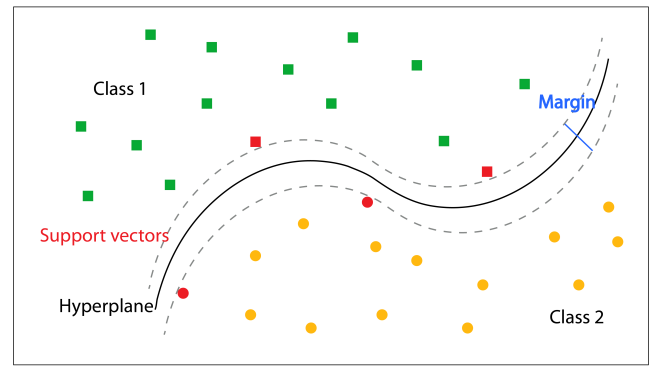

(b)

Figure 3.8: Illustration of separating two classes using SVM method. (a) Linear hyperplane. (b) Soft hyperplane [109].

After the application of these two tested classifiers, a post-processing stage was implemented. This strategy allows the rectification of some misclassifications that were originated by other structures that may be present in the eye fundus, as hard-exudates or vessels, as illustrated in Figure 3.9. In fact, these structures originate shadows on the posterior layers creating patterns with different intensity and texture properties that are distinct to the retinal tissue and, therefore, that can 
be confused with non-DRT candidate columns [110]. To solve this issue by improving the results, we implemented an approach to join disconnected groups of DRT identifications. This strategy is based on the aggregation factor $(d)$, which unifies two consecutive DRT regions when the distance between two candidate areas is smaller than the $d$ factor, as represented in Figure 3.10. In those cases, we assume that both detections belong to the same DRT edema, correcting the nonDRT classified columns defined by the classifier. This approach allows the fusion of the DRT regions, adjusting better the results to the real scenario and, therefore, improving the efficiency of the system.

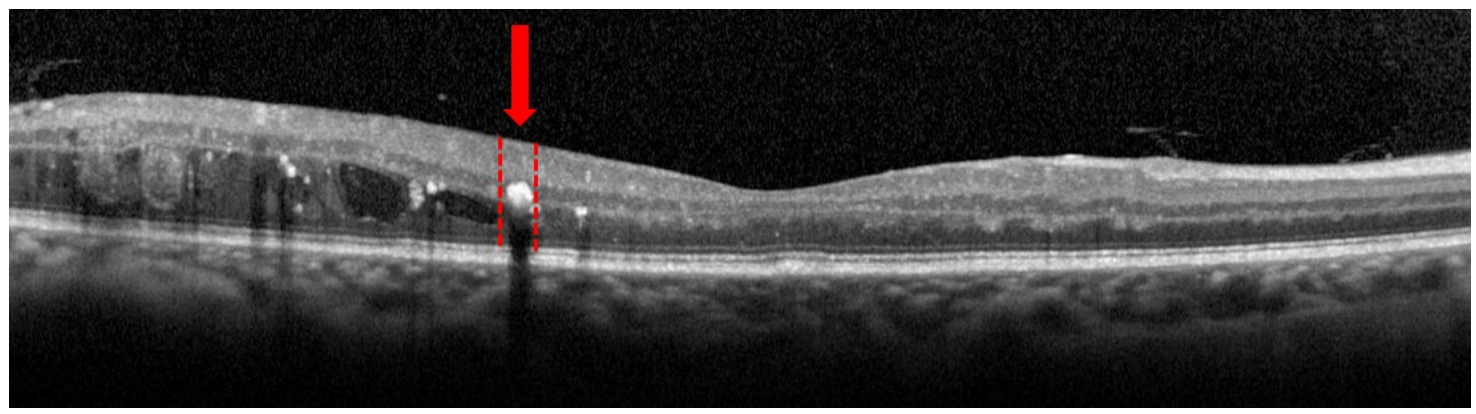

Figure 3.9: Example of OCT image with the presence of a hard-exudate in the above outer retina, producing a shadow in the lower layers.

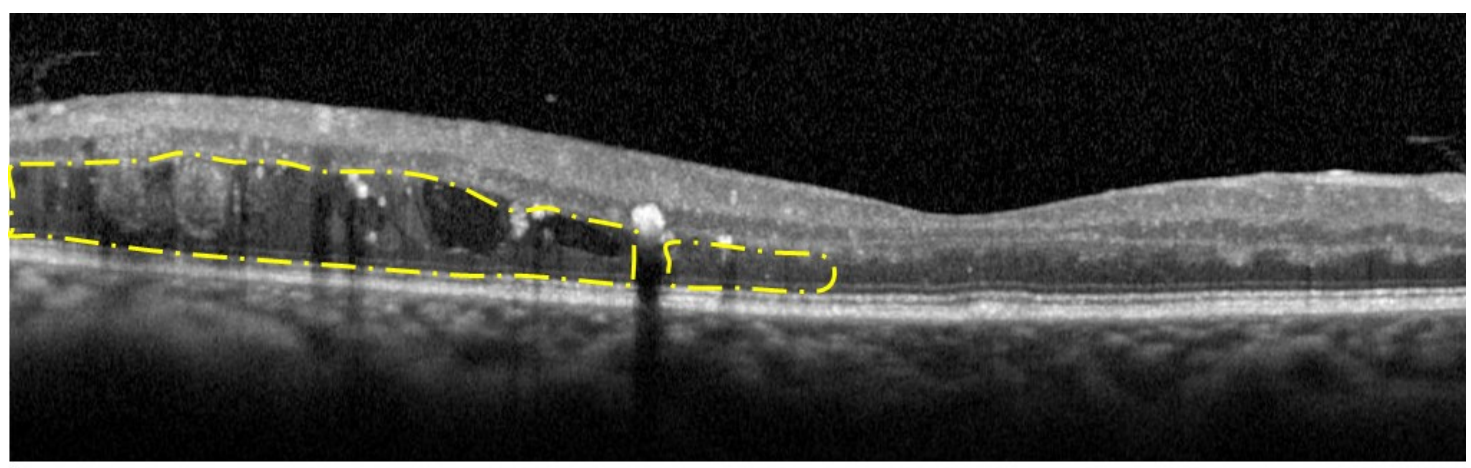

Aggregation Factor

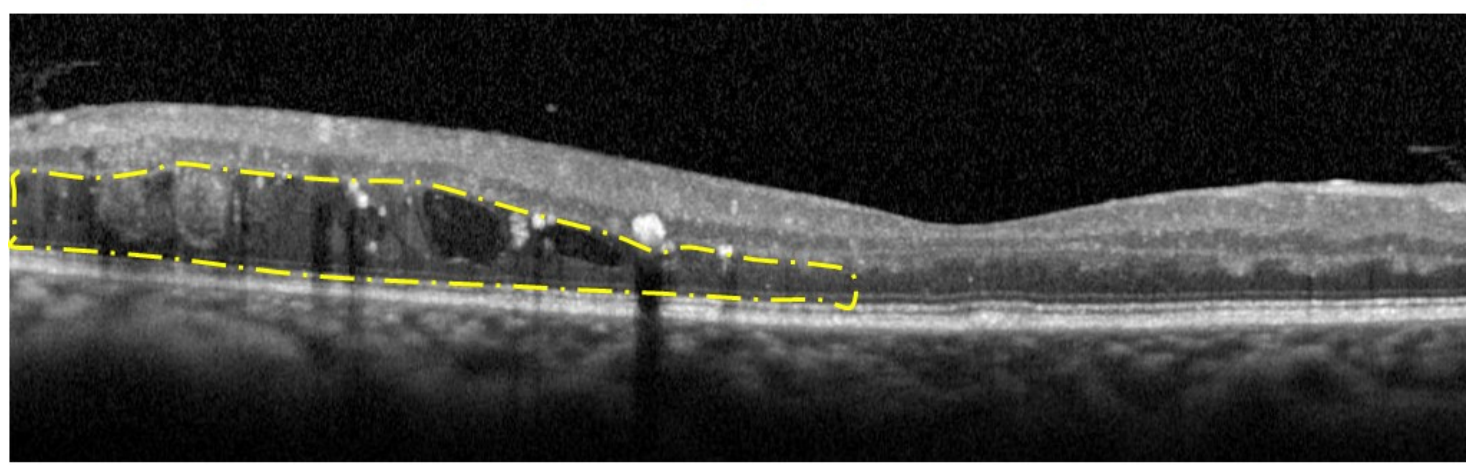

Figure 3.10: Example of OCT image with application of the aggregation factor $(d)$. 


\subsubsection{CME Detection}

CMEs are recognized as hyperreflective cystoid regions, as illustrated in Figure 3.11. Their morphology could vary from a honeycomb shape to a petaloid-like appearance (like petals of the flowers) [96]. This edema typically emerges in the inner retina with a significant contrast with the surrounded tissue. In more severe cases these edemas could migrate to the outer retina, where the contrast with the surrounded tissue is not so significant and the size of these edemas could vary considerably, as illustrated in Figure 3.12. Therefore, the presence of these edemas within the retinal tissue could lead to an increment of the normal retinal thickness.

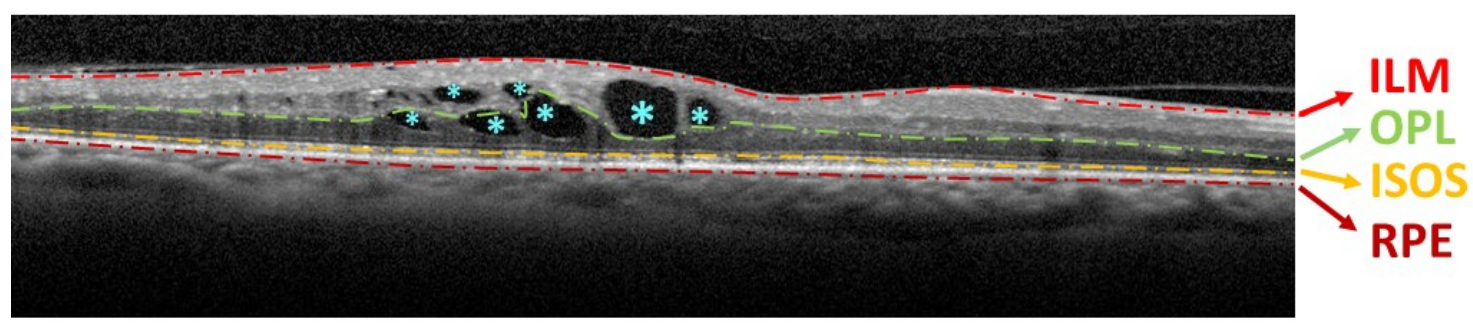

Figure 3.11: Example of OCT image with the presence of CME edemas $(*)$ in both retinal region (inner and outer retina).

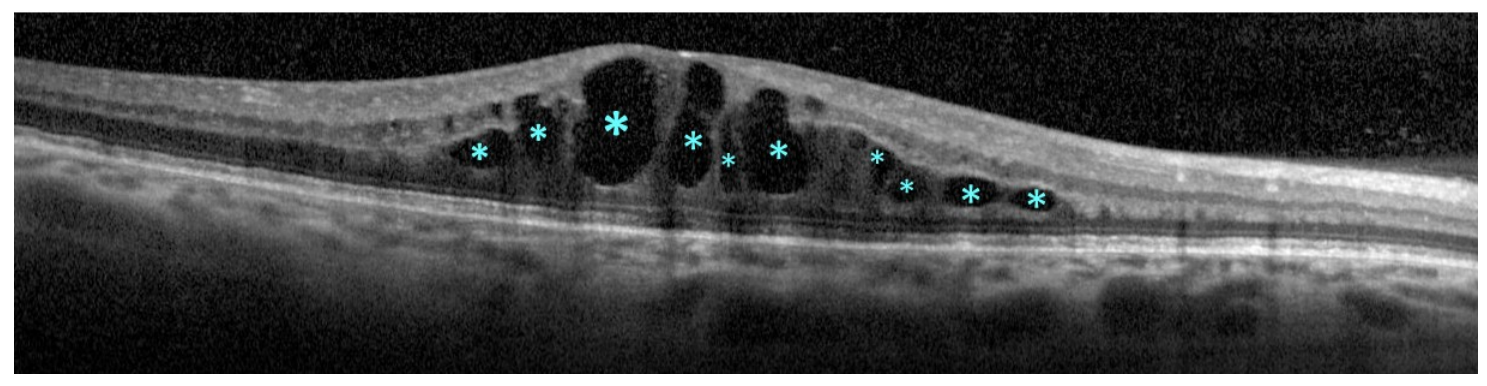

Figure 3.12: Example of OCT with the presence of CMEs $(*)$ in the outer retina with a variable size and morphology shapes.

The proposed system firstly searches the presence of these edemas in the inner retina and then in the outer retina. This strategy was adopted given that the contrast, as well as the characteristics of the CMEs in both regions, are slightly distinct. In the inner retina, CMEs typically present a well-defined cystoid shape and an evident contrast between the intraretinal fluid accumulation and the surrounding tissue. Then, as in the outer retina, these edemas present a poor contrast between the fluid region and the remaining retinal tissue, hardening their detection and representing a more challenging task. Figure 3.13 clearly illustrates this clinical pathological case, where the detection of these edemas in the outer retina represents a challenge due to the poor contrast between these cystoid regions and the neighborhood retinal tissue.

Regarding the typical elliptical shape and the low intensity profile of these edemas, we applied an adaptive threshold approach to identify the CMEs. Despite the adequate performance, this 


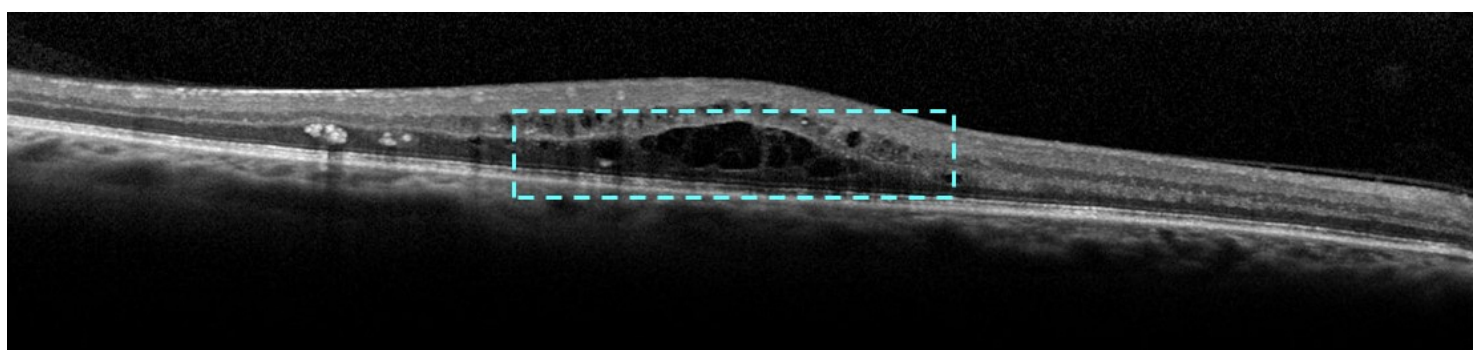

Figure 3.13: Example of OCT image with the presence of CMEs in the outer retina.

strategy presents some limitations as the possibility of production of partial segmentations of the same candidate cyst. In this way, it was applied a segmentation method based on an inundation process [111]. This post-processing stage enables the combination of two or more partial regions that belong to the same CME candidate as a way of obtaining more defined cystoid areas for the candidates. Subsequently, we analyzed the entire set of CME candidates to filter any existing false positive using a set of conditions derived from clinical references in the field [6, 112, 113, 114] and medical knowledge from the specialists participating in the work. The applied conditions in both regions are:

\section{- Area Size}

The restriction of the area size allows discarding candidates that are produced by noisy and tissue artifacts such as shadows from the vessels and hard-exudates, both commonly present in the retinal tissue. Therefore, we empirically determined the minimum and maximum areas to 5 pixels $s^{2}$ and 10 pixels $^{2}$ in the inner and outer retina, respectively. These minimum sizes were established considering the typical sizes that CMEs and noisy artifacts usually present in each region.

\section{- Major and Minor Axis of the Surrounding Ellipse}

CMEs are defined by the cystoid typical shapes that they normally present. Given this characteristic, each CME candidate is surrounded by an enclosing ellipse where it is determined by the minimum and maximum axis of the present ellipse. As limiting conditions, the minor and major lengths should be bigger than 7 and 200 pixels, respectively. These values were empirically determined as the minimum values that the CMEs usually present.

\section{- Retinal Thickness}

The presence of CMEs within the retinal tissue leads to an increment of the normal retinal thickness. In this way, to be considered as a candidate to CME, the retinal thickness in that region should be superior to $250 \mu \mathrm{m}$. 


\section{- Eccentricity}

As previously described, the shape of the CMEs could vary from a honeycomb to a petaloidlike appearance. Therefore, taking this shape variance as reference, we analyze the eccentricity of each candidate. In particular, this parameter measures the ratio between the central point of the ellipse and the major axis length of the major axis length, which should be smaller than 0.98 .

\subsection{Disease Characterization}

As previously explained, each ME type presents typical relative characteristics that are used by the specialists to recognize their presence in the OCT images. Despite these edemas are derived by the same cause, the leak of fluid from the capillaries, their characteristics and appearance within the retinal layers are significantly different. Given that, to facilitate the visual identification as well as to promote a quicker and more precise evaluation of the severity of the disease, the proposed system provides a labeled image as output. To aim this goal, from the three individual designed strategies, the partial identifications are combined resulting in a single output image where each ME type is identified with a specific symbol and color facilitating the revision and identification by the specialists.

\subsection{Properties of the Required Software}

The proposed methodology was entirely implemented using the MATLAB ${ }^{\circledR}$ software (MATrix LABoratory). It is designed as an interactive, high-performance numerical computing tool with a user-friendly environment and a large ability to perform tasks involving mathematical calculation in an interactive way (R2016a MATLAB ${ }^{\circledR}$ version).

This software was originally developed at the beginning of the 70's to solve problems related to matrix manipulations, algebra calculations and numerical analysis. Over the years, this computer program has been subject to successive forecast upgrades, with the launch of new features resulting in a more efficient performance when compared to the original version. This software is maintained and marketed through the company MathWorks, based in Massachusetts, United States. An advantage of this tool is that it provides a large set of functions, organized according to different scientific areas, designed as toolboxes. The programmer can enrich the MATLAB ${ }^{\circledR}$ by creating their own libraries of functions or extending the existing ones. There are currently many toolboxes that integrate MATLAB ${ }^{\circledR}$, such as:

- Symbolic Math Toolbox - functions for solving, plotting, and manipulating symbolic math equations;

- Signal Processing Toolbox - provides functions and apps to analyze, preprocess, and extract features from uniformly and non-uniformly sampled signals;

- Image Processing - provides a comprehensive set of reference-standard algorithms and workflow apps for image processing, analysis, visualization, and algorithm development; 
- Statistics and Machine Learning Toolbox - provides functions and apps to describe, analyze, and model data;

- Neural Network Toolbox (for Deep Learning) - Performance of neural networks topologies, and autoencoders for image classification, regression, and feature learning as well as their application for other strategies.

\subsection{Summary}

In this chapter, the computational methods that were adopted as well as the clinical criteria that was applied to the detection of each ME type were described. Despite the three types of ME (SRD, DRT and CME) are caused by a leak of fluid that is derived from the blood capillaries within the retinal tissue, they present a significant heterogeneity and variability among them. In this way, two main stages were designed to facilitate the identification and characterization of the three ME types: the identification of the region of interest and the identification of the different types of ME. Finally, this chapter also addressed the software that was required for the development of this project.

The first stage allows the identification of the ROI area, which limits the retinal tissue where the MEs are present. Subsequently, this region is sub-divided in 2 sub-regions: one corresponding to the inner retina and other to the outer retina. This strategy was designed to reduce the search area, once each type of ME have their typically relative position where they commonly appear in the retinal tissue.

As a second main stage of the methodology, the system identifies the different ME types by the implementation of three specific strategies in order to identify or not each faced ME type. Regarding the SRD and the CME edemas, as previously stated, their strategies are based on image processing techniques. In both cases, all the defined parameters were designed and determined in collaboration with the clinical specialists, using also well-established values from the clinical literature. Regarding the DRT edema, a machine learning strategy is proposed, once this edema presents a more complex appearance within the retinal tissue, with fuzzier and non-defined boundaries. To identify the presence of this edema, we propose a selection of 18 features, mainly based on texture or the corresponding increment in the retinal thickness, among others, as evidence of their presence. To determine the subset of features that better describe the DRT presence, the application of three feature selectors are proposed: SFS, RFS and SVM-FS; and two classifiers: Naive Bayes and SVM. This strategy allows the comparison between the different combinations in order to determine which one provides the best performance for the DRT detection. Finally, the proposed methodology combines the three strategies to provide the final output image result to the specialists to facilitate their inspection.

Finally, a brief description of the software required for the development of this project was reported. 


\section{Chapter 4}

\section{Results}

In this chapter, the results obtained from the implementation of the proposed methodology are presented. Initially, the dataset that was used in this project and some important characteristics are described, also, some particularities of implementation of this study are enumerated. In the following sections the results for the previously described steps are presented, namely, the region of interest identification and the extraction of the different types of ME.

For the development of this proposed project, an ASUS G501VW Series was used with an Intel ${ }^{\circledR}$ i7-6700HQ CPU with a $2.59 \mathrm{GHz}$ processor, $16 \mathrm{~GB}$ of RAM memory, NVIDIA ${ }^{\circledR}$ GeForce ${ }^{\circledR}$ 960M graphical card and 1TB hard drive. It runs a Windows 10 Home 64-bit operating system, which is compatible with the computational tool used by MATALAB ${ }^{\circledR}$.

This experimental study was performed using the R2016a MATLAB ${ }^{\circledR}$ version and different toolboxes as the symbolic math toolbox, the statistics and machine learning toolbox and the image processing toolbox.

The dataset of OCT images used in this study was obtained from a SD-OCT imaging with OCT - Spectralis ${ }^{\circledR}$ OCT confocal scanning laser ophthalmoscope from Heidelberg Engineering. The OCT scans were obtained using a configuration of a 7 Line Rater scan with a $30^{\circ} \times 5^{\circ}$ of the angle of capture and with a space of $240 \mu \mathrm{m}$ apart from each other. In this study, we used a total of 170 OCT images centered in the macula, with a resolution of $2,032 \times 596$ pixels.

Regarding the clinical information about the used dataset, the images were obtained from adult diabetic patients of both genders (M/F) between 2016/2018. Additionally, the OCT images are from both left and right eyes of different individuals, presenting a varying degree of ME pathology. In particular, many appearances of the three ME types can simultaneously appear in a single scan. To ensure the anonymity of the patients that participated in this study, the corresponding images were renamed by the ophthalmologists before being provided for the system validation. The local ethics committee approved the study and the tenets of the Declaration of Helsinki were followed.

The system is composed by two main stages: delimitation of the region of interest and identification of all the appearances of the different ME types. Firstly, the proposed system identifies two regions: the inner and the outer retina. Then, within these previously identified regions, three 
fully automatic strategies were implemented to individually detect the three types of ME using the OCT images.

The precise identification of each retinal layer as well as the presence and characterization of the three ME types present as main limitations the typical complex characteristics of the OCT images such as the lack of tissue details, the speckle noise that is frequently present in this image modality or the poor soft tissue contrast in some acquisition scenarios.

For the identification of the Region of Interest, the system firstly identifies the limiting layers of the retinal tissue: the ILM and the RPE layers as superior and inferior boundaries, respectively, by the application of Chiu et al. [93] method.

Given the alternative proposal for the OPL layer extraction, to measure the efficiency of this designed stage, an expert clinician labeled 20 representative OCT images, 10 pathological and 10 non-pathological images, both from the present dataset. For this analysis, two retinal layers were labeled by a specialist: the OPL and the RPE layers. These segmented layers are used to quantify the OPL layer extraction and, consequently, the outer retina identification. A total of 47,136 marked points were obtained from both retinal layers. To measure the performance of this proposal, three statistical metrics were implemented that were used in similar validation processes [115]: Mean Absolute Difference (MAD), Correlation Coefficient (CC) and Dice Coefficient (DC) (Equations 4.1, 4.2 and 4.3, respectively). The MAD metric analyzes the absolute mean difference, in pixels, between both segmentations of the OPL layer (manual and proposed method). The CC metric determines the correlation between them. Finally, the DC metric compares the similarity between both segmentations of the entire outer retina, which is delimited by the OPL and the RPE layers as superior and inferior boundaries, respectively.

$$
\begin{gathered}
M A D=\frac{1}{N} \sum_{k=1}^{N} m_{i}-p_{i} \\
C C=\frac{\sum_{k=1}^{N} m_{i} p_{i}}{\sqrt{\sum_{k=1}^{N} m_{i}^{2} \sum_{k=1}^{N} p_{i}^{2}}} \\
D C=\frac{2 \sum_{k=1}^{N}\left|m_{i} \cap p_{i}\right|}{\sum_{k=1}^{N}\left|m_{i}\right|+\sum_{k=1}^{N}\left|p_{i}\right|}
\end{gathered}
$$

where $m_{i}$ and $p_{i}$ represent the manual detection and the output of the proposed method, respectively, at the $\mathrm{i}^{\text {th }}(\mathrm{i}=1 \ldots \mathrm{N})$ column in two measurements of the OCT image dimensions.

The results obtained by the proposed system for the OPL layer and the outer retina identification are listed in Table 4.1. As a global performance in non-pathological and pathological cases, the proposed system achieves values of 1.51 pixels for MAD, $99.97 \%$ of CC and 0.9439 of DC metrics. 
Table 4.1: Obtained results for the OPL layer and outer retina identifications in non-pathological and pathological OCT images.

\begin{tabular}{|c|c|c|c|}
\hline Cases & MAD (pixels) & CC & DC \\
\hline Non-pathological & 1.951 & $99.96 \%$ & 0.9623 \\
\hline Pathological & 1.077 & $99.98 \%$ & 0.9255 \\
\hline Global & 1.514 & $99.97 \%$ & 0.9439 \\
\hline
\end{tabular}

Regarding the edema identification process, in each of the extracted regions, the inner and the outer retina, the system identifies all the existing edemas of the three ME types in terms of locations and amount. Given that, to validate the proposed methodology, an expert clinician labeled the images indicating the regions with the presence of the three types of ME.

In the case of the SRD and CME extraction approaches, we directly compared the identification of the system with the specialist annotations. In the DRT case, we used the expert labeling by columns, as reference, to construct the training and test sets of the implemented learning strategy. This stage was validated using the following metrics: Precision, Recall, Accuracy and F-Measure. Mathematically, these metrics are formulated as indicated in Equations 4.4, 4.5, 4.6 and 4.7, respectively. F-Measure is defined as a combination of both precision and recall metrics in a global measurement of the performance of the system.

$$
\begin{gathered}
\text { Precision }=\frac{T P}{T P+F P} \\
\text { Recall }=\frac{T P}{T P+F N} \\
\text { Accuracy }=\frac{T P+T N}{T P+T N+F P+F N} \\
F-\text { Measure }=2 * \frac{\text { Precision } * \text { Recall }}{\text { Precision }+ \text { Recall }}
\end{gathered}
$$

As gold standard, we considered for the validation of the SRD and CME extraction:

- True Positive (TP): we verified if the central point of each detected edema matches any identification of the specialist. In the case of two or more candidates matching the same manual identification, we only considered the first candidate to ensure the method efficiency.

- False Positive (FP): if the central point does not overlap any manual identification of the specialist.

- False Negative (FN): if any manual labeling of the specialist is not covered with any central point provided by the system.

- True Negative (TN): otherwise. Given the significative unbalance between both positive and negative sets, TNs are not considered with these edema types. 
In the case of the DRT edemas, the same analysis is performed by columns, where we used, as gold standard:

- True Positive (TP): if a column indicated by the ophthalmologist with DRT presence was classified as DRT by the proposed system.

- False Positive (FP): if a column indicated by the ophthalmologist with non-DRT presence was classified as DRT by the proposed system.

- False Negative (FN): if a column indicated by the ophthalmologist with DRT presence was classified as non-DRT by the proposed system.

- True Negative (TN): if a column indicated by the ophthalmologist without DRT presence was classified as non-DRT by the proposed system.

SRD case is a particular and less frequent type of ME. In fact, these edemas only affect a reduced group of patients [63] and, in case of existing, only one occurs per OCT image. For that reason, the used dataset of 170 OCT images only contains 10 SRD edemas. We would like to remark that the 10 cases were correctly identified by the proposed system.

Regarding the DRT detection, a learning strategy was applied using both the Naive Bayes and SVM classifiers. For that, we used a window size with a width of 5 columns, value empirically determined, and a variable height summing a total of 5,600 samples extracted from both DRT and non-DRT regions to train and test the classifiers without any preprocessing or application of correction parameters. This dataset was divided into two subsets, one for training and the other for testing. Next, to ensure the global performance of the method, we trained both classifiers using a 10-fold cross-validation using 10 repetitions, being the mean error/accuracy calculated to determine the most suitable configuration for each case using the best performance. Then, using the best configuration of each classifier, we proceed with the corresponding validation processes using the test dataset. We tested the performance of the system with 51,346 columns containing DRT and 68,976 non-DRT edemas.

Firstly, to detect the DRT edemas, three feature selectors (RFS, SVM-FS and SFS) were applied to determine the subset of features that better discriminate the presence of this ME type. The majority of the selected features were taken from the intensity image analysis, mask thickness and GLCM texture-based features, as they present the highest capacity of differentiation between DRT and non-DRT patterns.

Table 4.2 lists the performances in the test dataset for each classifier and the optimal subsets of each analyzed feature selector. The Naive Bayes classifier obtained the best configuration, reaching an accuracy of $87.45 \%$ using the RFS selected subset, whereas the SVM classifier obtained an accuracy of $86.14 \%$ using the SFS selected subset.

Secondly, as listed in Table 4.3, we analyzed the performance achieved by both classifiers, using a window width of 5 columns. In fact, both classifiers presented a satisfactory behavior in the detection of DRT edemas, being the Naive Bayes classifier slightly better, reaching a FMeasure of $84.45 \%$. 
Table 4.2: Obtained results by the analyzed classifiers and the selected feature subsets.

\begin{tabular}{|c|c|c|c|}
\hline Classifier & Selector & F-Measure & Accuracy \\
\hline \multirow{3}{*}{ Naive Bayes } & RFS & $\mathbf{8 4 . 4 5 \%}$ & $\mathbf{8 7 . 4 9 \%}$ \\
\cline { 2 - 4 } & SVM-FS & $84.39 \%$ & $87.44 \%$ \\
\cline { 2 - 4 } & SFS & $84.36 \%$ & $87.41 \%$ \\
\hline \multirow{3}{*}{ SVM } & RFS & $82.72 \%$ & $86.10 \%$ \\
\cline { 2 - 4 } & SVM-FS & $82.75 \%$ & $86.12 \%$ \\
\cline { 2 - 4 } & SFS & $\mathbf{8 2 . 7 9 \%}$ & $\mathbf{8 6 . 1 4 \%}$ \\
\hline
\end{tabular}

Table 4.3: Obtained results for the DRT detection using the best configurations of the Naive Bayes and SVM classifiers using the testing dataset.

\begin{tabular}{|c|c|c|c|}
\hline Classifier & Precision & Recall & F-Measure \\
\hline Naive Bayes & $89.88 \%$ & $79.65 \%$ & $84.45 \%$ \\
\hline SVM & $88.09 \%$ & $78.08 \%$ & $82.79 \%$ \\
\hline
\end{tabular}

Finally, we tested the use of the aggregation factor, $d$, to merge isolated candidates of the same DRT regions. Figure 4.1 shows the obtained F-Measures in the calculation of the optimal aggregation factor using both classifiers. More detailed information is listed in Table 4.4, where the best aggregation factor for the Naive Bayes classifier is $d=72$, whereas for the SVM classifier is $d=21$, reaching F-Measures of $87.54 \%$ and $85.22 \%$, respectively.

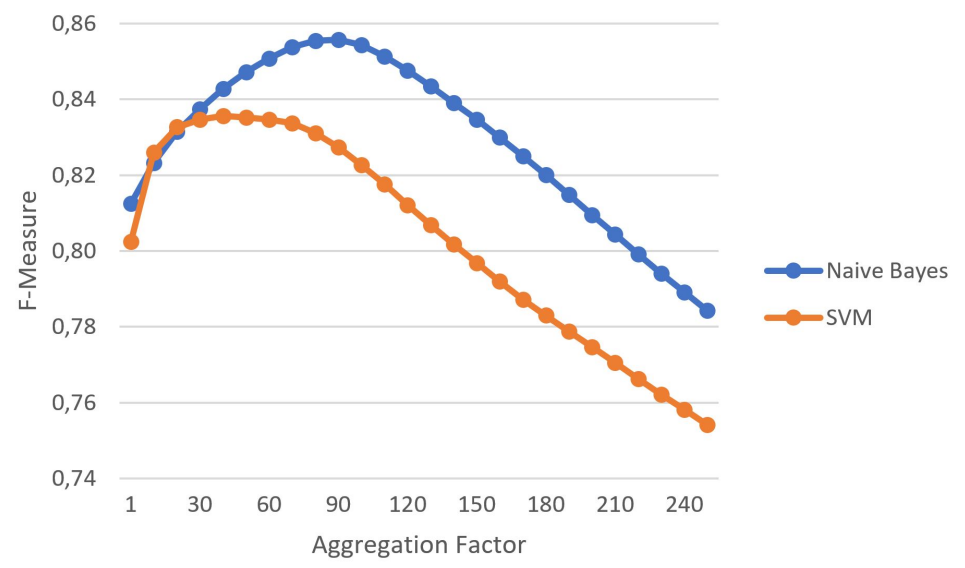

Figure 4.1: Determination of the optimal aggregation factor. Obtained F-Measures.

Figure 4.2 illustrates an output of the proposal of the best aggregation factor that unifies single candidates in grouped "sponge-like" edema detections. 
Table 4.4: F-Measures that were obtained with the tested classifiers and their corresponding optimal aggregation factors using the testing dataset.

\begin{tabular}{|c|c|c|c|c|}
\hline Classifier & $\begin{array}{c}\text { Aggregation } \\
\text { Factor }\end{array}$ & Precision & Recall & F-Measure \\
\hline Naive Bayes & $d=72$ & $85.65 \%$ & $89.51 \%$ & $87.54 \%$ \\
\hline SVM & $d=21$ & $81.94 \%$ & $88.78 \%$ & $85.22 \%$ \\
\hline
\end{tabular}
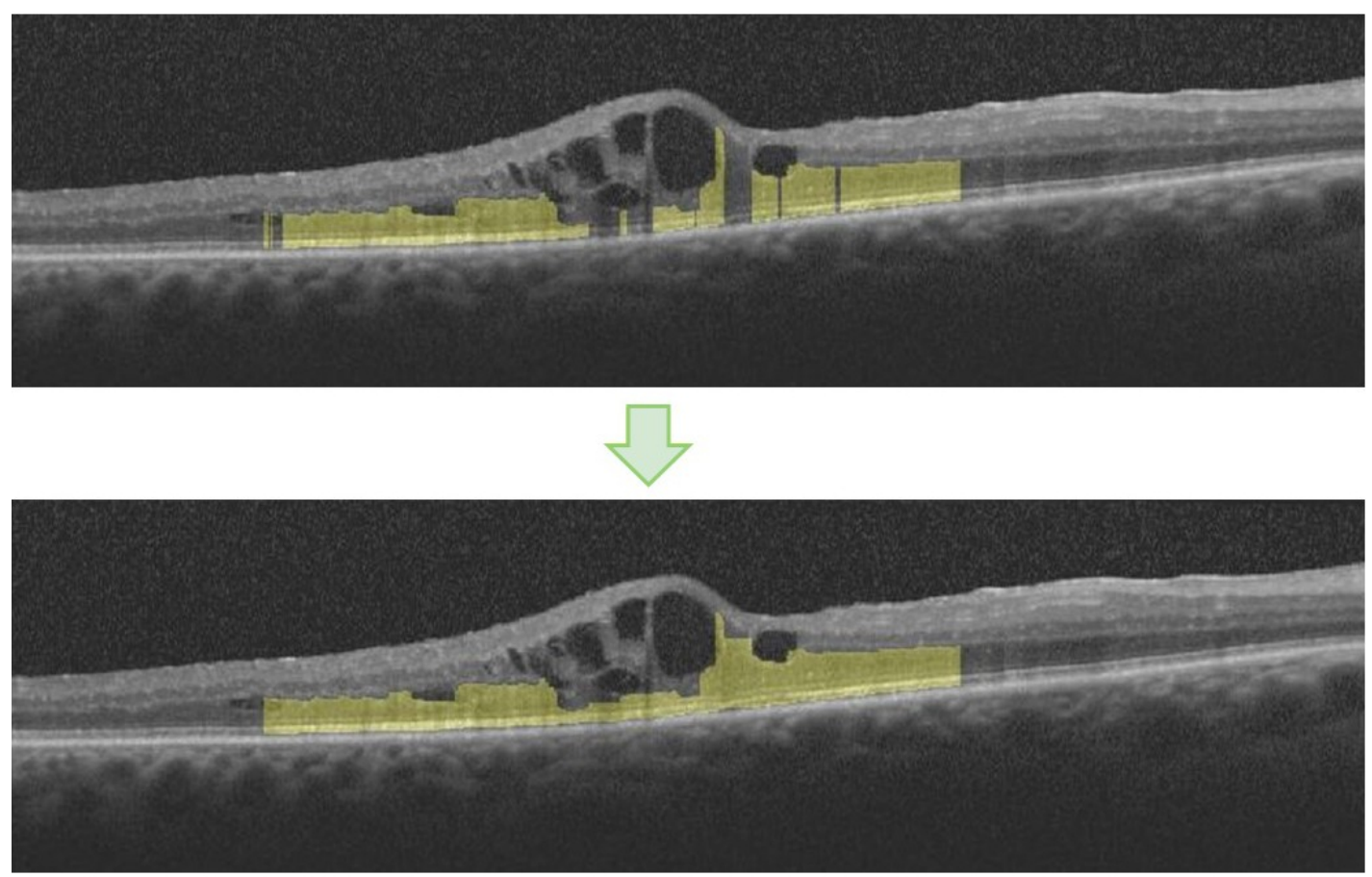

Figure 4.2: OCT image with a DRT edema presence in the outer retina. The application of the aggregation factor unifies single candidates in a grouped "sponge-like" edema detection.

Regarding the CME detections, we established two main steps to detect the presence of CMEs in the inner and outer retina, individually. We tested the performance of the system with 379 CMEs that were manually identified by the specialist in the entire image dataset.

The results obtained are summarized in Table 4.5, where F-Measure reached values of 96.08\% and $81.65 \%$ in the inner and outer retina, respectively. Finally, as global performance, the system obtained an accurate F-Measure of $91.99 \%$.

Table 4.5: Results of the CME detection using Precision, Recall and F-Measure.

\begin{tabular}{|c|c|c|c|}
\hline Region & Precision & Recall & F-Measure \\
\hline Inner Retina (ILM/OPL) & $97.03 \%$ & $95.15 \%$ & $96.08 \%$ \\
\hline Outer Retina (OPL/RPE) & $74.17 \%$ & $90.82 \%$ & $81.65 \%$ \\
\hline ROI (Inner and Outer Retina) & $90.13 \%$ & $93.93 \%$ & $91.99 \%$ \\
\hline
\end{tabular}




\section{Chapter 5}

\section{Discussion}

OCT images have become an important auxiliary tool in clinical practice routines and scientific studies, helping the clinicians in the diagnosis and evaluation of the severity of many diseases.

Given that, we propose a system that firstly segments the inner and the outer retina. In each of these identified regions, three fully automatic strategies were implemented to individually identify the three types of the ME disease. This strategy was validated using the clinical references in the field as well as the knowledge of the specialist that incorporates this research study.

Generally, this issue presents as main limitation the complex typical characteristics typical of the OCT images such as the lack of tissue details, the speckle noise or the poor soft tissue contrast in some capture scenarios.

Regarding the identification of the upper limiting membrane of the outer retina, the OPL layer, we quantitatively compared the results of our approach with the performance of the work proposed by Chiu et al. [93]. The decision of using this alternative approach was motivated by the poor results of the extraction of the OPL layer by the original method in advanced pathological images that, in our case, represent the most frequent scenario. Given that, we compared the performance of both methods in non-pathological and pathological OCT images. As illustrated in Figure 5.1, both methods present a similar performance for the identification of the OPL layer and the outer retina in non-pathological OCT images (Figure 5.1 (a), (c) \& (e)). However, in the case of pathological OCT images, the performance of the proposed system is significantly better in comparison with the original method.

Figure 5.2 illustrates these situations with examples of both non-pathological and pathological OCT images. As we can see, in accordance with the metrics, both methods present a favorable performance for the OPL layer extraction in non-pathological OCT images (Figure 5.2 (a) \& (b)). However, in pathological images, the original method provides poor results of the OPL layer, crossing the entire macular region (Figure 5.2 (c) \& (e)). In opposition, our proposal detects efficiently this layer (Figure 5.2 (d) \& (f)), facilitating the posterior division of the retinal layers in the consequent inner and outer retina. The precise contour of the macular region facilitates the identification of the DRT and the CME edemas. In the particular case of the DRT edemas, the thickness of the outer retina, as well as the ROI, is a determinant feature to identify its presence 


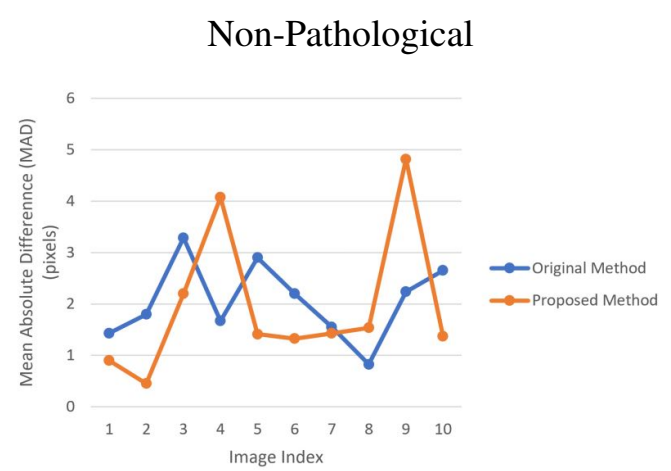

(a)

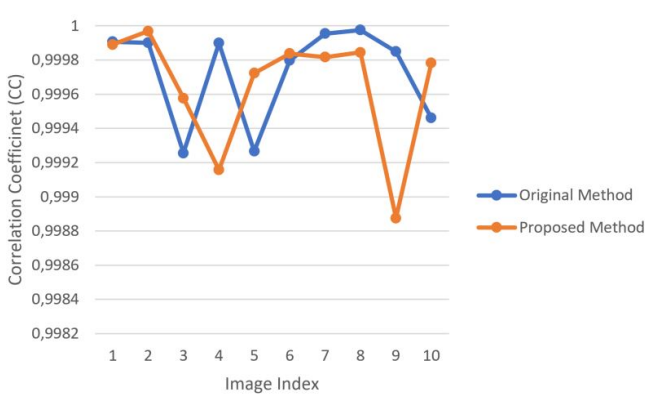

(c)

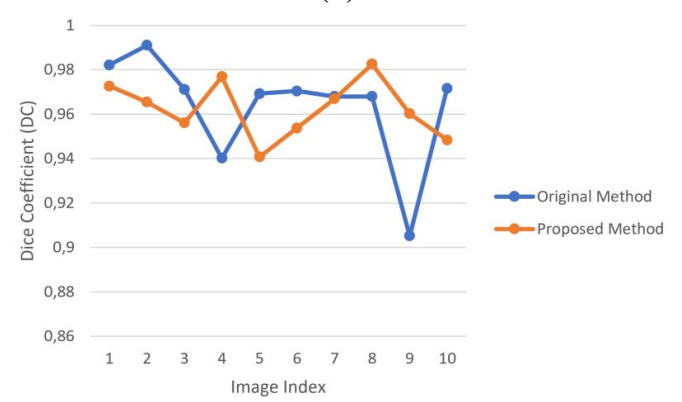

(e)

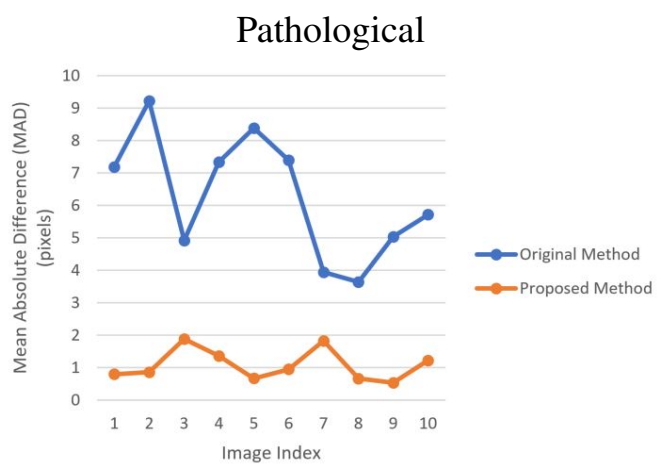

(b)

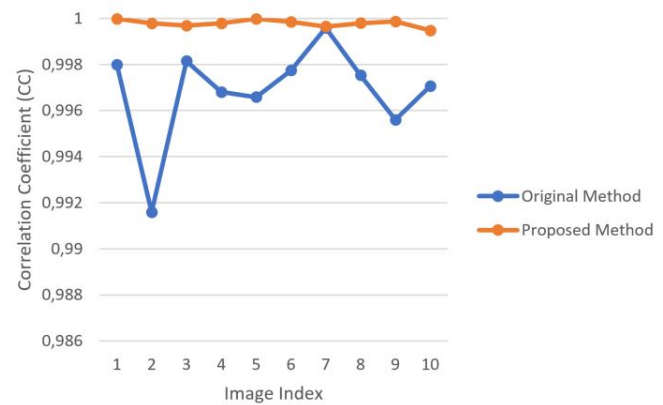

(d)

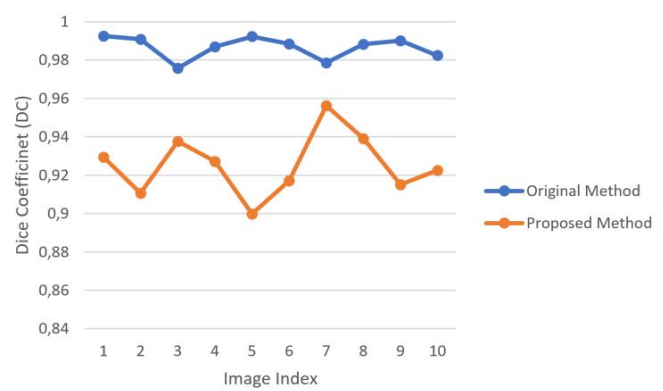

(f)

Figure 5.1: Comparative analysis between the performance of the original method and our proposal for the OPL layer and the outer retina identifications, using non-pathological ( $1^{\text {st }}$ column) and pathological cases $\left(2^{\text {nd }}\right.$ column). (a) \& (b) Using MAD metric over the OPL extraction. (c) \& (d) Using CC metric over the OPL extraction. (e) \& (f) Using DC metric over the entire outer retina segmentation.

in the OCT images. Additionally, CMEs present different intensity profiles in the inner and outer retina, which facilitates their identification.

Despite the high variability and complexity of the ME disease, the proposed system using the implemented specific strategies is capable of detecting efficiently the hypothetical presence of the three types of ME, even when they appear simultaneously in the same OCT image. Each of these strategies works independently with respect to the others, identifying each faced ME type. The output binary identifications of the three strategies are finally combined to provide the final image result to the specialists to ease their inspection, as illustrated in Figure 5.3. 


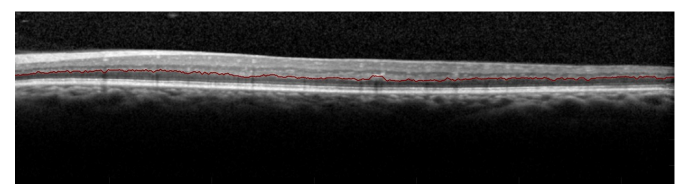

(a)

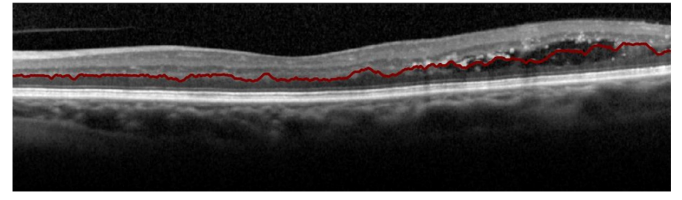

(c)

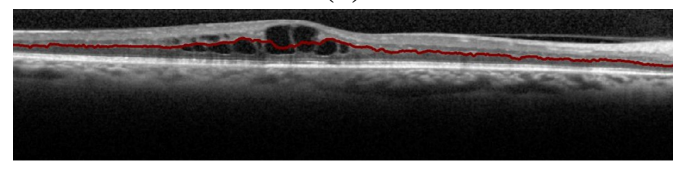

(e)

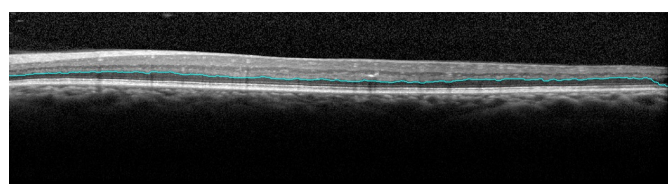

(b)

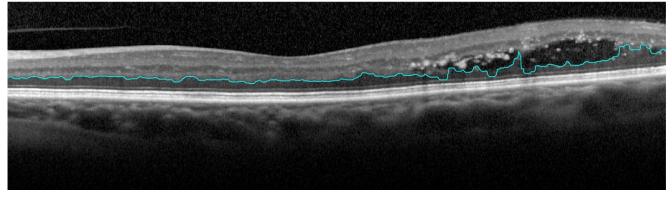

(d)

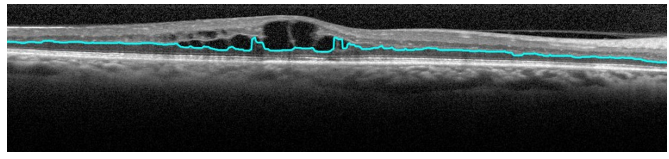

(f)

Figure 5.2: Example of OCT images with the OPL identification from the output of the original method and our proposal. $1^{\text {st }}$ row, non-pathological OCT images. $2^{\text {nd }} \& 3^{\text {rd }}$ rows, pathological OCT images.

SRD edemas are a particular case that does not appear frequently. In this case, the typical contrast of the photoreceptors layer is similar to the presence of this ME type in early stages, which difficult its identification in some cases. Even in such cases, the system performed the SRD identifications correctly. Similar works of the literature also detect all the SRD cases [80, 77, 74]. However, this comparison presents some limitations, given that each study was made using their own image dataset.

In the case of the DRT edemas, to date, none scientific proposal studied the issue of the automatic detection and identification of this ME type. This challenge is mainly associated to the absence of limiting membranes as well as the significant pattern variability. Consequently, these limitations make more difficult the detection of the retinal regions that are affected by this ME type. Given that, a learning strategy was applied to detect the presence of this edema in the outer retina. In the validation performance, three feature selectors were used: RFS, SVM-FS and SFS. Also, two classifiers were analyzed: Naive Bayes and SVM. Regarding the selected features, the majority of them were taken from the intensity image analysis, mask thickness and GLCM texture-based features. These features are in concordance with the characteristics of the DRT edemas, once they are characterized with "sponge-like" patterns with a significant variability in terms of intensities. Given that, features such as maximum, mean, variance and standard deviation from the global intensity features and the correlation from the GLCM features were mostly selected. Additionally, the thickness of the ILM/RPE and the ratio between the OPL/RPE and ILM/RPE layers were also selected in the first positions as the DRT presence typically implies not only an increment of the outer retinal thickness but also the increment of the entire "retinal thickening".

Using the previously selected features, both classifiers were trained and tested using the best configuration. Comparing the results of Table 4.3 and Table 4.4, we can conclude that the appli- 
cation of the aggregation factor increases significantly the accuracy in the case of the Naive Bayes classifier, as illustrated in the example of Figure 4.2. In the case of the SVM classifier, this increment was lower. Generally, this difference is given by the SVM classifier, which is more sensitive to noise. For that reason, it produces sets of candidate columns of a variable size in both non-DRT and DRT regions. Instead of that, Naive Bayes presents a considerable number of consecutive candidate columns in the DRT region with a small distance between them. In this situation, the use of the aggregation factor is crucial, merging this small separated DRT detections in coherent merged ones and being this case the one that obtained a higher improvement of this step of the method. This factor benefits the Naive Bayes classifier by connecting significantly close DRT detections whereas this penalizes the SVM case by merging non-DRT with DRT regions. With large values, the aggregation factor groups regions that are too far from each other, decreasing progressively the obtained results.

Regarding the CME detections, we defined two individual main steps to detect the presence of CMEs in the inner and outer retina. Firstly, the system detects these edemas in the inner retina (ILM/OPL), where they present a higher contrast and well-defined boundaries. These characteristics facilitate the identification of the fluid regions. For that reason, the performance of the system in this first region is significantly better. Despite the large variability in terms of size and shape, the implemented approach successfully identified the CMEs, as shown in Figure 5.3. In fact, the proposed system was capable to detect even cystoid edemas with complex petaloid shapes that appear often merged even between both regions.
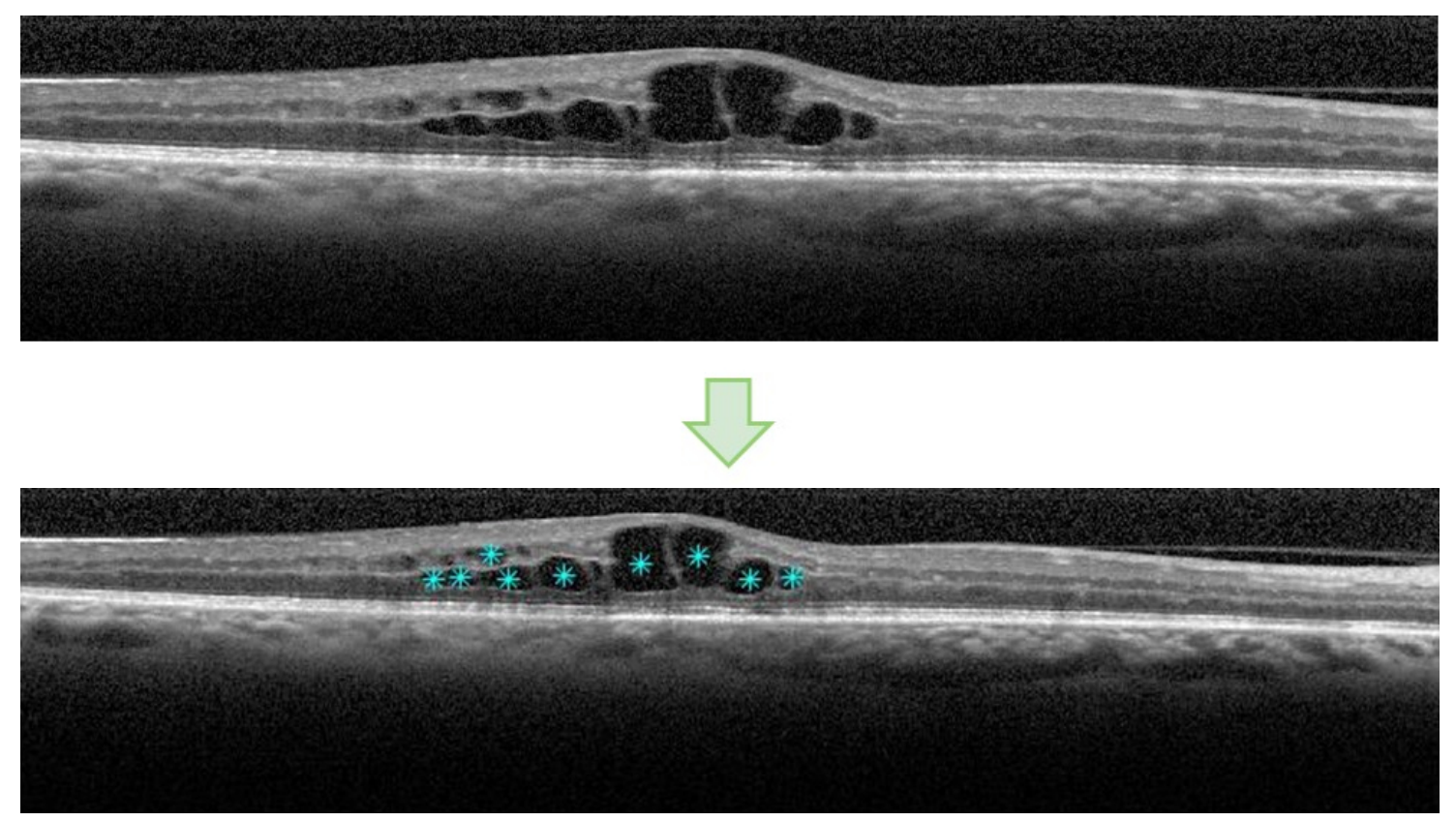

Figure 5.3: Example of OCT image with the presence of CMEs (*) in both retinal regions.

Most of the missed CMEs present specific and uncommon morphologies and sizes, such as those with fusiform shapes or cystoid edemas with reduced areas. Figure 5.4 presents an illus- 
trative example of the first case with the presence of a cyst with an unusual horizontal elliptical shape.

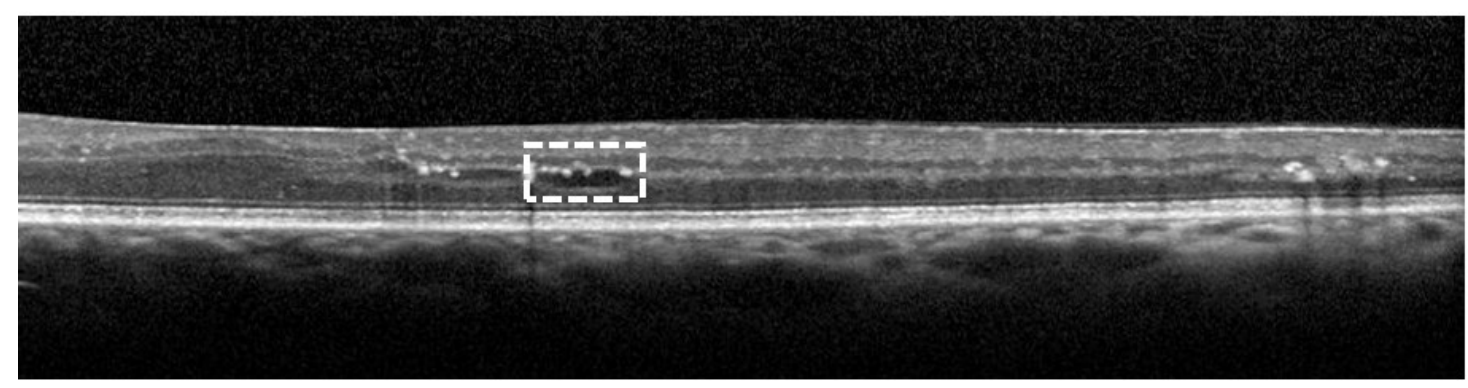

Figure 5.4: Example of OCT image with a non-detected CME (- -), with an elliptical horizontal shape in the inner retina.

In the case of small edemas, they are easily mistaken with noise and other artifacts that are typically present in the OCT images. In fact, as explained, the system removes small identifications considering the sizes that noisy and artifact detections typically present in the inner and outer retina. This restriction also avoids the detection of any present microcyst, being out of the scope of this work.

Moreover, the presence of fuzzy contours further complicates the detection of these edemas. Additionally, when these edemas are proliferated in nearby groups, their individual identification is more complex to accomplish.

Secondly, the performance of the system was tested in the outer retina. As indicated, in this region, CMEs do not present well-defined boundaries, being frequently mistaken with the surrounded tissues given the almost absent contrast between these fluid accumulations and the neighboring retinal tissues. Moreover, many times, in advanced pathological cases, CMEs are surrounded by the presence of DRT edemas, hardening the identification of these cases. Consequently, the accuracy of the method is decreased in this region but still with a satisfactory performance (Table 4.5).

Summarizing, we proposed a system for the individual identification of the three types of ME that is also capable of their identification when they appear simultaneously in the OCT scans. This is illustrated in Figure 5.5 with examples of the individual and the merged presence of the different ME types. Firstly, we can see satisfactory individual identifications of SRD edemas (Figure 5.5 (a) \& (b)), DRT edemas (Figure 5.5 (c) \& (d)) and CME edemas (Figure 5.5 (e) \& (f)). We also present the simultaneous detection of DRT and CME cases (Figure $5.5(\mathrm{~g}) \&(\mathrm{~h})$ ) where we can see that the proposed system is capable to identify them even if they appear merged. This idea is also presented in the representative case of Figure 5.6 with a scan with ME presence in an advanced stage. In this case, we can see the accurate performance of the system with the simultaneous detection of the three types that are present in the retinal layers in this single scan. 


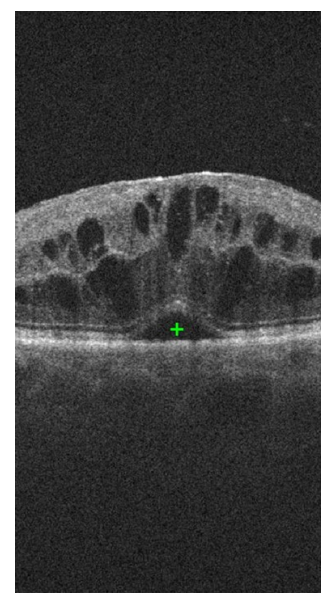

(a)

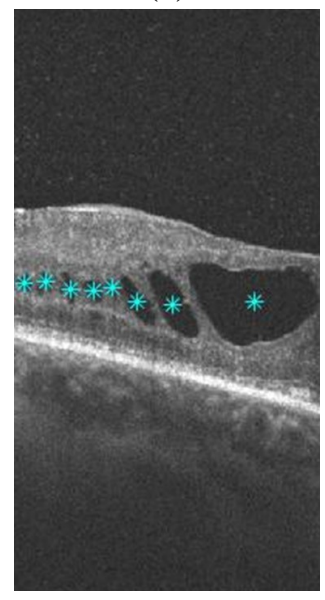

(e)

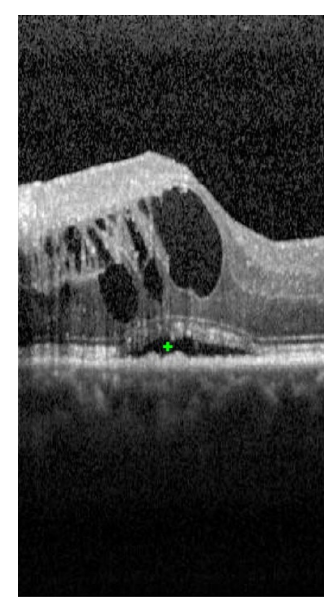

(b)

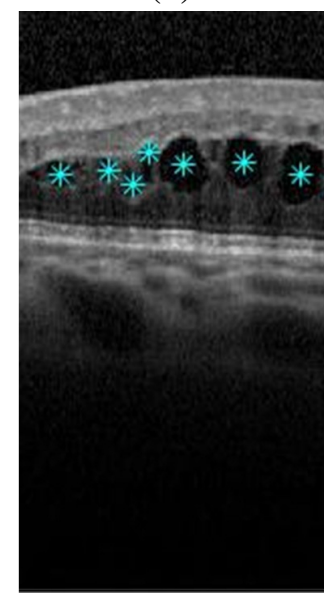

(f)

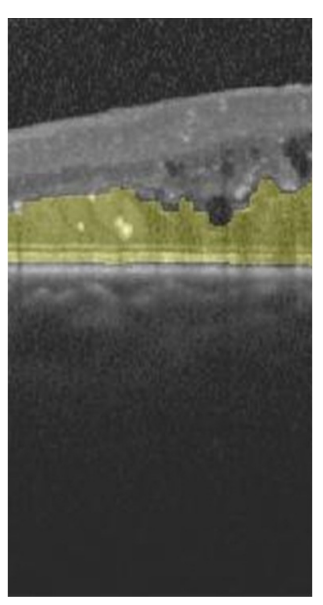

(c)

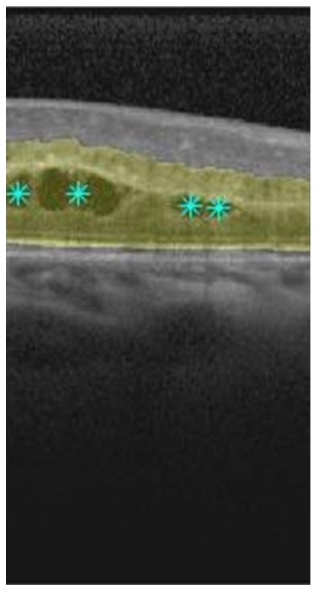

(g)

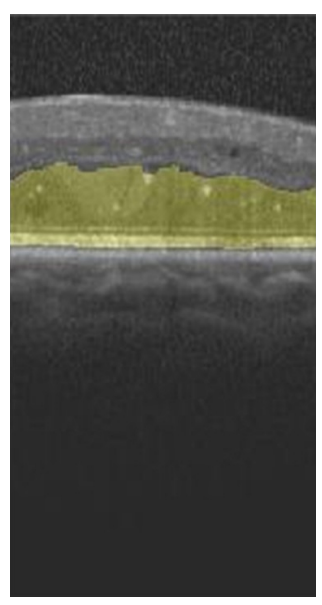

(d)

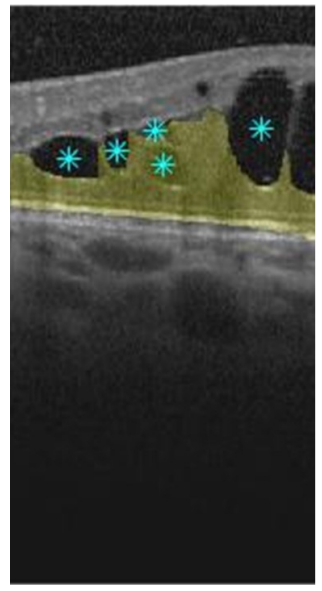

(h)

Figure 5.5: Examples of ME detections in OCT images: (a) \& (b) SRD identification (+). (c) \& (d) DRT regional identification. (e) \& (f) CME detections (*). (g) \& (h) Simultaneous identification of CMEs $(*)$ and DRT.

Table 5.1 lists a comparative analysis using two literary works of reference that faced the CME detection and our proposed strategy. This comparison presents some limitations, given that each proposal used specific private datasets, without any specifications about the selected cases that were used in the study, limiting the comparison among the different analyzed approaches. As we can see, our method offers a competitive performance with respect to the other proposals. 

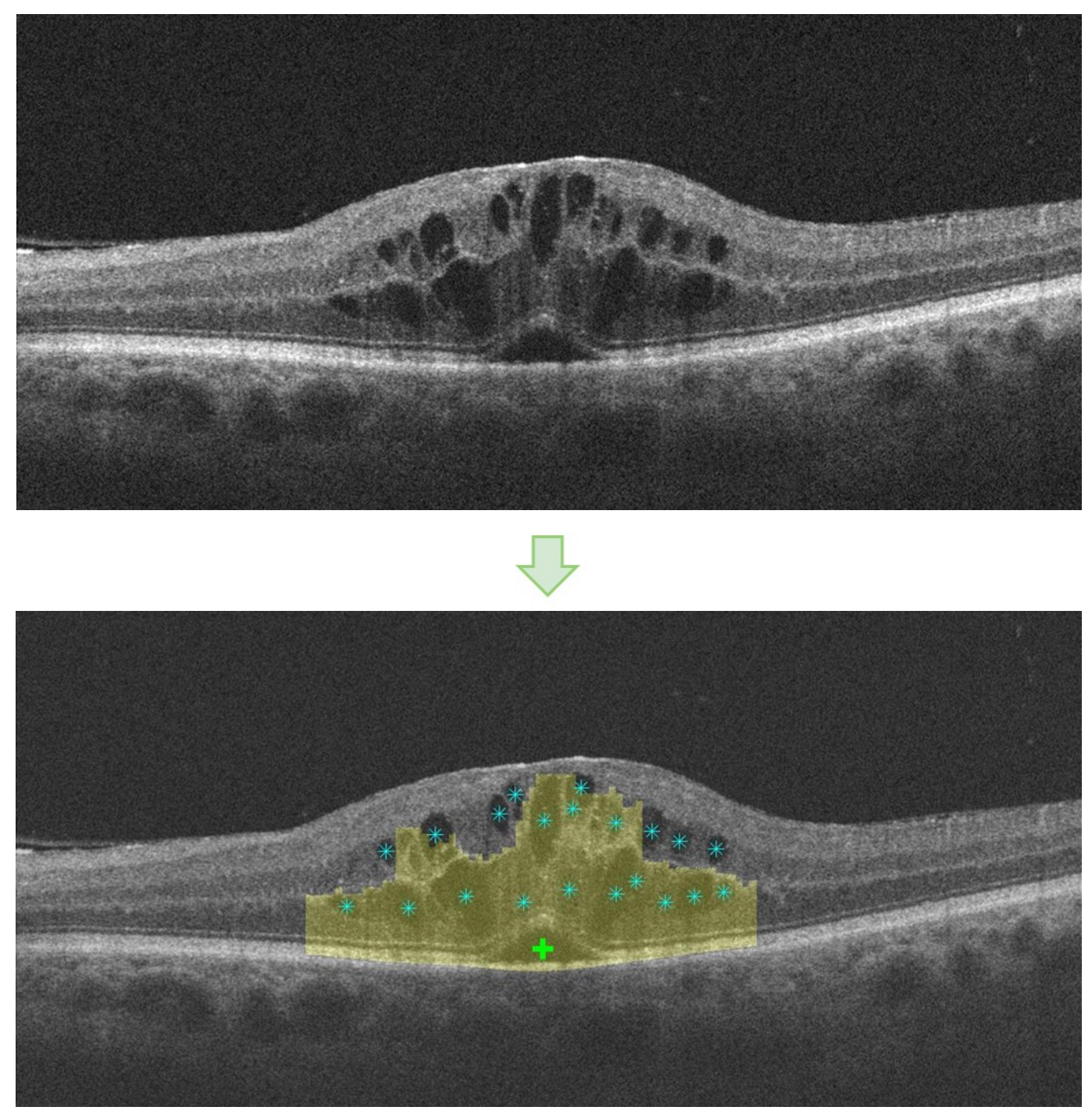

Figure 5.6: Example of OCT image with the detection of the three types of MEs: SRD illustrated as (+), CME as (*) and DRT.

Table 5.1: CME detection performance comparison of state of the art approaches and the proposed method.

\begin{tabular}{|c|c|c|c|}
\hline Method & Precision & Recall & F-Measure \\
\hline Schlegl et al. [80] & $99.00 \%$ & $89.00 \%$ & $93.73 \%$ \\
\hline González et al. [84] & $80.00 \%$ & $77.11 \%$ & $78.53 \%$ \\
\hline Proposed & $90.13 \%$ & $93.93 \%$ & $91.99 \%$ \\
\hline
\end{tabular}




\section{Chapter 6}

\section{Conclusions}

The present dissertation proposes a complete methodology for the analysis and the characterization of the ME presence. This fully automatic system detects and characterizes, using OCT images, the three types of ME that may appear in the eye fundus of the patients using OCT images. Given that, the main goal of this project was the study, analysis and development of an auxiliary computational tool that helps in the diagnosis and clinical decisions procedures.

This work was developed under the Erasmus+ Internship program in the host VARPA Research Group of the Faculty of Computer Science (FIC) - University of A Coruña, Spain. The project is enclosed in a multidisciplinary and collaborative research line that involves clinical and technological specialists from different scientific areas as medical imaging, ophthalmology and engineering. Furthermore, the research line followed in this project was supported by Spanish and Galician (regional) competitive projects.

Using the information provided by the OCT scans, image processing techniques were combined with learning strategies and complemented with clinical knowledge to construct a robust system that accurately aimed the identification of the three types of ME.

Regarding the sub-division of the ROI in the inner and the outer retina, the system efficiently detects the OPL layer in both cases, considering pathological and non-pathological scenarios. This strategy was specially designed for the OPL layer and the outer retina identifications in pathological OCT images. In this stage of the methodology, it was obtained satisfactory results, reaching an approximated value of 1 for the MAD metric and 0.99 for CC and DC metrics in pathological images.

A similar performance was obtained in the automatic detection and characterization of the three ME types. In the case of CMEs, the system achieved a global F-Measure of $91.99 \%$ in both retinal regions, while the identification of the DRT presence reached a value of $87.54 \%$, using the best combination of Naive Bayes classifier and RFS selector. In the case of the SRD edemas, the system was capable to adequately detect all the cases that appear in the used dataset.

Summarizing, despite the high variability and complexity of this retinal pathology, the proposed system is capable to accurately identify all the types of ME, representing a useful auxiliary tool, that aids the clinicians in this complex and exhausting work of detection and characterization 
of a relevant disease as is ME. Given the novelty and the relevance of the work, a manuscript publication was recently published in the Journal Computer Methods and Programs in Biomedicine (Journal citation, first quartile) [116].

As future works plan, we aim to extend the methodology with more sophisticated procedures, especially in the case of CMEs in the outer retina, as well as include further features and classifiers for the DRT detection. Also, the methodology is planned for being expanded with an automatic detection of MME, providing further medical information for the early diagnosis of ME. To cover the entire characterization of the ME disease, we aim to extend the proposed methodology by its precise segmentation. In addition, further validations could be implemented by the increase of the dataset dimensionality, which enables also the application of other ambitions techniques in this problematic, as deep learning strategies. Therefore, it will be possible to follow more precisely the evolution of the disease and improve, consequently, the life quality of the patients. 


\section{Appendix A}

\section{Publications}

\section{A.1 Journals}

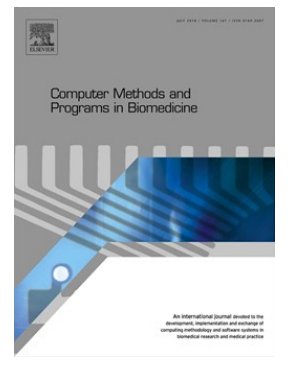

G. Samagaio, A. Estévez, J. de Moura, J. Novo, M. Fernandez and M. Ortega. Automatic Macular Edema Identification and Characterization Using OCT Images. Computer Methods and Programs in Biomedicine, 2018.

\section{A.2 Conferences}

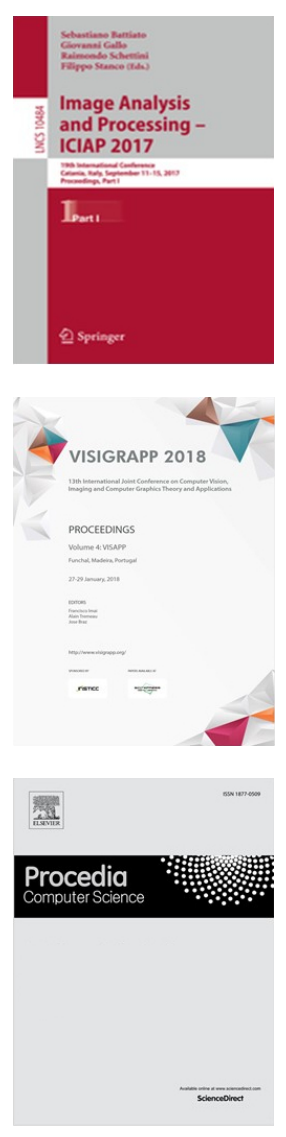

G. Samagaio, J. de Moura, J. Novo and M. Ortega. Optical Coherence Tomography Denoising by Means of a Fourier Butterworth Filter-Based Approach. In International Conference on Image Analysis and Processing, Springer, Cham. pp. 422-432, 2017.

G. Samagaio, A. Estévez, J. de Moura, J. Novo and M. Ortega. Automatic Identification of Macular Edema in Optical Coherence Tomography Images. VISIGRAPP'18. 14th International Joint Conference on Computer Vision, Imaging and Computer Graphics Theory and Applications, 4:533-540, 2018.

(Accepted) G. Samagaio, J. de Moura, J. Novo and M. Ortega. Automatic Segmentation of Diffuse Retinal Thickening Edemas Using Optical Coherence Tomography Images. 22nd International Conference on Knowledge-Based and Intelligent Information \& Engineering Systems, 2018. 


\section{References}

[1] Diabetic retinopathy, May 2018. URL: https://www.mayoclinic.org/ diseases-conditions/diabetic-retinopathy/symptoms-causes/ syc-20371611.

[2] Eye Care. URL: http://www.who.int/pbd/blindness/ WorldSightDay17Infographic.pdf?ua=1.

[3] Vision Impairment and Blindness. URL: http://www.who.int/mediacentre/ factsheets/fs282/en/.

[4] R. Bourne, S. Flaxman, T. Braithwaite, M. Cicinelli, A. Das, J. Keeffe, and et al. Magnitude, Temporal Trends, and Projections of the Global Prevalence of Blindness and Distance and Near Vision Impairment: A Systematic Review and Meta-Analysis. Lancet Glob Health, 5(9):e888-e897, 2017.

[5] P. de Azevedo-Marques, A. Mencattini, M. Salmeri, and R. Rangayyan. Medical Image Analysis and Informatics: Computer-Aided Diagnosis and Therapy. CRC Press, 2017.

[6] J. Gass and E. Norton. Cystoid Macular Edema and Papilledema Following Cataract Extraction: A Fluorescein Fundoscopic and Angiographic Study. Archives of Ophthalmology, 76(5):646-661, 1966.

[7] A. Joussen, T. Gardner, B. Kirchhof, and S. Ryan. Retinal Vascular Disease, volume 75. Springer, 2007.

[8] M. Nelson and A. Martidis. Managing Cystoid Macular Edema After Cataract Surgery. Current Opinion in Ophthalmology, 14(1):39-43, 2003.

[9] Rhys Williams, M Airey, H Baxter, J\&kennedy-martin Forrester, T Kennedy-Martin, and A Girach. Epidemiology of Diabetic Retinopathy and Macular Oedema: A Systematic Review. Eye, 18(10):963, 2004.

[10] João Figueira, L Gonçalves, José Henriques, et al. Retinopatia Diabética Guidelines. Sociedade Portuguesa de Oftalmologia, 2009.

[11] Sociedade Portuguesa Diabetologia. Diabetes: Factos e Números, Relatório Anual do Observatório Nacional da Diabetes, 2013.

[12] José Henriques, Sara Vaz-Pereira, João Nascimento, and Paulo Caldeira Rosa. Diabetic Eye Disease. Acta Medica Portuguesa, 28(1):107-113, 2015.

[13] J. Pevsner. Leonardo da Vinci's Contributions to Neuroscience. Trends in Neurosciences, 25(4):217-220, 2002. 
[14] K. Irsch and D. Guyton. Anatomy of Eyes., 2009.

[15] M. Hardy and S. Heyes. Beginning Psychology. Oxford University Press, 1999.

[16] E. Hodges. The Guild Handbook of Scientific Illustration. John Wiley \& Sons, 2003.

[17] H. Ellis and V. Mahadevan. Clinical Anatomy: Applied Anatomy for Students and Junior Doctors. John Wiley \& Sons, 2010.

[18] A. Mescher. Junqueira's Basic Histology: Text and Atlas. Mcgraw-hill, 2013.

[19] G. Hildebrand and A. Fielder. Anatomy and Physiology of the Retina. In Pediatric Retina, pages 39-65. Springer, 2011.

[20] D. Purves, G. Augustine, D. Fitzpatrick, et al. Cones and Color Vision, 2001.

[21] D. Purves, G. Augustine, D. Fitzpatrick, L. Katz, A. LaMantia, J. McNamara, and S. Williams. Neuroscience 2nd Edition. Sunderland (MA) Sinauer Associates, 2001.

[22] Biology- Sensory System. URL: https://cnx.org/contents/GFy_h8cud10.53: fVwAeW4E@6/Vision.

[23] S. Malavade. Overview of the Ophthalmic System. In Nano-Biomaterials For Ophthalmic Drug Delivery, pages 9-36. Springer, 2016.

[24] Lee A. Remington. Clinical Anatomy of the Visual System E-Book. Elsevier Health Sciences, 2011.

[25] How the Retina Allows Us To See, Retina Doctor Chevy Chase, Jan 2017. URL: https://www.washingtoneye.com/retina/ understanding-retina-allows-us-see/

[26] B. Wandell. Foundations of Vision. Sinauer Associates, 1995.

[27] J. Forrester, A. Dick, P. McMenamin, F. Roberts, and E. Pearlman. The Eye E-Book: Basic Sciences in Practice. Elsevier Health Sciences, 2015.

[28] C Contet, SP Goulding, DA Kuljis, and AL Barth. Bk channels in the central nervous system. In International review of neurobiology, volume 128, pages 281-342. Elsevier, 2016.

[29] T. Neumann. Clinical Anatomy and Physiology of the Visual System. Clinical Anatomy, $1: 2,2016$.

[30] S. Omri, B. Omri, M. Savoldelli, L. Jonet, B. Thillaye-Goldenberg, G. Thuret, and et al. The Outer Limiting Membrane (OLM) Revisited: Clinical Implications. Clinical Ophthalmology (Auckland, NZ), 4:183, 2010.

[31] C. Phelps. Introduction to Ophthalmoscopy. Archives of Ophthalmology, 95(6):1079-1079, 1977.

[32] C. Ruete. Der Augenspiegel u. des Optometer für Practische Aerzte. 1852.

[33] R. Keeler. The Ophthalmoscope in the Lifetime of Hermann von Helmholtz. Archives of Ophthalmology, 120(2):194-201, 2002. 
[34] C. Wood. The Fundus Oculi of Birds: Especially as Viewed by the Ophthalmoscope: A Study in the Comparative Anatomy and Physiology. Lakeside Press, 1917.

[35] G. Timberlake and M. Kennedy. The Direct Ophthalmoscope How It Works and How to Use It, 2005.

[36] I. Cordero. Understanding and Caring for the Direct Ophthalmoscope. Community Eye Health, 29(93):17, 2016.

[37] Bruce James and Larry Benjamin. E-Book-Ophthalmology: Investigation and Examination Techniques. Elsevier Health Sciences, 2006.

[38] Liebreich Richard. Richard Liebreich (1830-1917) e o Primeiro Atlas de Oftalmoscopia. Revista da AMRIGS, 54(3):356-359, 2010.

[39] Ismael Cordero. Understanding and Caring for an ndirect Ophthalmoscope. Community Eye Health, 29(95):57, 2016.

[40] HEINE Optotechnik. Heine omega $₫ 500$ binocular indirect ophthalmoscope. URL: https://www.heine.com/en_GB/ specialties/ophthalmology/product/product-detail/ 23908-heine-omegaR500-binocular-indirect-ophthalmoscope/.

[41] D. Huang, E. A Swanson, C. Lin, J. S Schuman, W. Stinson, W. Chang, M. Hee, T. Flotte, K. Gregory, C. A Puliafito, et al. Optical Coherence Tomography. Science (New York, NY), 254(5035):1178, 1991.

[42] A. Podoleanu. Optical Coherence Tomography. Journal of Microscopy, 247(3):209-219, 2012.

[43] A. A Michelson and E. Morley. On the Relative Motion of the Earth and of the Luminiferous Ether. Sidereal Messenger, vol. 6, pp. 306-310, 6:306-310, 1887.

[44] C. Nucci, L. Cerulli, N. Osborne, and G. Bagetta. Glaucoma: An Open-Window to Neurodegeneration and Neuroprotection, volume 173. Newnes, 2008.

[45] P. Jansz, S. Richardson, G. Wild, and S. Hinckley. Biomedical Image Signal Processing for Reflection-Based Imaging. In Biomedical Engineering-Technical Applications in Medicine. InTech, 2012.

[46] Zeiss Cirrus HD. URL: http://www.cirrusdemo.com//.

[47] Radha Das, Gareth Spence, Ruth E Hogg, Michael Stevenson, and Usha Chakravarthy. Disorganization of Inner Retina and Outer Retinal Morphology in Diabetic Macular Edema. JAMA ophthalmology, 2018.

[48] G. Trichonas and P. Kaiser. Optical Coherence Tomography Imaging of Macular Oedema. British Journal of Ophthalmology, 98(Suppl 2):ii24-ii29, 2014.

[49] Diabetic retinopathy: The signs, symptoms, and solutions. URL: https://ocularpro. com/diabetic-retinopathy-the-signs-symptoms-and-solutions/.

[50] Direção Geral de Saúde. Programa Nacional para a Diabetes 2017, 2017. URL: https: //www.dgs.pt/programa-nacional-para-a-diabetes.aspx 
[51] Sociedade Portuguesa de Oftalmologia. Oftalmologia. REVISTA DA SOCIEDADE PORTUGUESA DE OFTALMOLOGIA, 34(4), 2015.

[52] Q. Mohamed, A. Ross, and C. Chu. Diabetic Retinopathy (Treatment). BMJ Clinical Evidence, 2011, 2011.

[53] Diabetic Retinopathy Treatment, Oct 2017. URL: https://www.aao.org/ eye-health/diseases/diabetic-retinopathy-treatment.

[54] S. Pershing, E. Enns, B. Matesic, D. Owens, and J. Goldhaber-Fiebert. Cost-Effectiveness of Treatment of Diabetic Macular Edema. Annals of Internal Medicine, 160(1):18-29, 2014.

[55] What Is Macular Degeneration?, Jan 2018. URL: https://www.aao.org/ eye-health/diseases/amd-macular-degeneration.

[56] L. Mogk and M. Mogk. Macular Degeneration: The Complete Guide to Saving and Maximizing Your Sight. Ballantine Books, 2003.

[57] J. Sunness. The Natural History of Geographic Atrophy, the Advanced Atrophic Form of Age-Related Macular Degeneration. Mol Vis, 5(25):25, 1999.

[58] I. Wong, S. Koo, and C. Chan. Prevention of Age-Related Macular Degeneration. International Ophthalmology, 31(1):73-82, 2011.

[59] Facts About Age-Related Macular Degeneration, Sep 2015. URL: https://nei.nih. gov/health/maculardegen/armd_facts.

[60] Age-Related Macular Degeneration (AMD). URL:https://nei.nih.gov/eyedata/ amd\#3.

[61] Diabetic Retinopathy. URL:https://nei.nih.gov/eyedata/diabetic

[62] Tratamiento de la Retinopatía Proliferativa. URL: http://www.diabetesforecast. org/2015/may-jun/es/tratamiento-de-la-retinopata.html.

[63] T. Otani, S. Kishi, and Y. Maruyama. Patterns of Diabetic Macular Edema with Optical Coherence Tomography. American Journal of Ophthalmology, 127(6):688-693, 1999.

[64] G. Panozzo, B. Parolini, E. Gusson, A. Mercanti, S. Pinackatt, G. Bertoldo, and S. Pignatto. Diabetic Macular Edema: An OCT-Based Classification. In Seminars in Ophthalmology, volume 19, pages 13-20. Taylor \& Francis, 2004.

[65] K. Seo, S. Yu, M. Kim, and H. Kwak. Visual and Morphologic Outcomes of Intravitreal Ranibizumab for Diabetic Macular Edema based on Optical Coherence Tomography Patterns. Retina, 36(3):588-595, 2016.

[66] H Joel Trussell and Michael J Vrhel. Fundamentals of Digital Imaging. Cambridge University Press, 2008.

[67] Desire Sidibe, Shrinivasan Sankar, Guillaume Lemaitre, Mojdeh Rastgoo, Joan Massich, Carol Cheung, Gavin Tan, Dan Milea, Ecosse Lamoureux, Tien Wong, et al. An Anomaly Detection Approach for the Identification of DME Patients Using Spectral Domain Optical Coherence Tomography Images. Computer Methods and Programs in Biomedicine, 139:109-117, 2017. 
[68] Cecilia S Lee, Ariel J Tyring, Nicolaas P Deruyter, Yue Wu, Ariel Rokem, and Aaron Y Lee. Deep-learning based, automated segmentation of macular edema in optical coherence tomography. Biomedical optics express, 8(7):3440-3448, 2017.

[69] Abhijit Roy, Sailesh Conjeti, Sri Phani Karri, Debdoot Sheet, Amin Katouzian, Christian Wachinger, and Nassir Navab. Relaynet: Retinal Layer and Fluid Segmentation of Macular Optical Coherence Tomography Using Fully Convolutional Network. Biomedical optics express, 8(8):3627-3642, 2017.

[70] K. Alsaih, G. Lemaitre, M. Rastgoo, J. Massich, D. Sidibé, and F. Meriaudeau. Machine Learning Techniques for Diabetic Macular Edema (DME) Classification on SD-OCT Images. Biomedical Engineering Online, 16(1):68, 2017.

[71] N. Dalal and B. Triggs. Histograms of Oriented Gradients for Human Detection. In Computer Vision and Pattern Recognition, 2005. CVPR 2005. IEEE Computer Society Conference on, volume 1, pages 886-893. IEEE, 2005.

[72] T. Ojala, M. Pietikainen, and T. Maenpaa. Multiresolution Gray-Scale and Rotation Invariant Texture Classification with Local Binary Patterns. IEEE Transactions on Pattern Analysis and Machine Intelligence, 24(7):971-987, 2002.

[73] A. Montuoro, S. Waldstein, B. Gerendas, U. Schmidt-Erfurth, and H. Bogunović. Joint Retinal Layer and Fluid Segmentation in OCT Scans of Eyes with Severe Macular Edema Using Unsupervised Representation and Auto-Context. Biomedical Optics Express, 8(3):1874-1888, 2017.

[74] Zhuli Sun, Haoyu Chen, Fei Shi, Lirong Wang, Weifang Zhu, Dehui Xiang, Chenglin Yan, Liang Li, and Xinjian Chen. An Automated Framework for 3D Serous Pigment Epithelium Detachment Segmentation in SD-OCT Images. Scientific Reports, 6:21739, 2016.

[75] B. Hassan, G. Raja, T. Hassan, and U. Akram. Structure Tensor Based Automated Detection of Macular Edema and Central Serous Retinopathy Using Optical Coherence Tomography Images. JOSA A, 33(4):455-463, 2016.

[76] Jelena Novosel, Ziyuan Wang, Henk de Jong, Mirjam Van Velthoven, Koenraad Vermeer, and Lucas van Vliet. Locally-Adaptive Loosely-Coupled Level Sets for Retinal Layer and Fluid Segmentation in Subjects With Central Serous Retinopathy. In Biomedical Imaging (ISBI), 2016 IEEE 13th International Symposium, pages 702-705. IEEE, 2016.

[77] M. Wu, W. Fan, Q. Chen, Z. Du, X. Li, S. Yuan, and H. Park. Three-Dimensional Continuous Max Flow Optimization-Based Serous Retinal Detachment Segmentation in SD-OCT for Central Serous Chorioretinopathy. Biomedical Optics Express, 8(9):4257-4274, 2017.

[78] M. Wu, Q. Chen, X. He, P. Li, W. Fan, S. Yuan, and H. Park. Automatic Subretinal Fluid Segmentation of Retinal SD-OCT Images with Neurosensory Retinal Detachment Guided by Enface Fundus Imaging. IEEE Transactions on Biomedical Engineering, 65(1):87-95, 2018.

[79] A. Syed, T. Hassan, U. Akram, S. Naz, and S. Khalid. Automated Diagnosis of Macular Edema and Central Serous Retinopathy Through Robust Reconstruction of 3D Retinal Surfaces. Computer Methods and Programs in Biomedicine, 137:1-10, 2016. 
[80] T. Schlegl, S. Waldstein, H. Bogunovic, F. Endstraßer, A. Sadeghipour, A. Philip, and et al. Fully Automated Detection and Quantification of Macular Fluid in OCT Using Deep Learning. Ophthalmology, 2017.

[81] Donghuan Lu, Morgan Heisler, Sieun Lee, Gavin Ding, Marinko Sarunic, and Mirza Beg. Retinal Fluid Segmentation and Detection in Optical Coherence Tomography Images using Fully Convolutional Neural Network. CoRR 1710.04778, 2017.

[82] G. Wilkins, O. Houghton, and A. Oldenburg. Automated Segmentation of Intraretinal Cystoid Fluid in Optical Coherence Tomography. IEEE Transactions on Biomedical Engineering, 59(4):1109-1114, 2012.

[83] Sohini Roychowdhury, Dara Koozekanani, Salma Radwan, and Keshab Parhi. Automated Localization of Cysts in Diabetic Macular Edema Using Optical Coherence Tomography Images. In Engineering in Medicine and Biology Society (EMBC), 2013 35th Annual International Conference of the IEEE, pages 1426-1429. IEEE, 2013.

[84] A. González, B. Remeseiro, M. Ortega, M. Penedo, and P. Charlón. Automatic Cyst Detection in OCT Retinal Images Combining Region Flooding and Texture Analysis. In Computer-Based Medical Systems (CBMS), pages 397-400. IEEE, 2013.

[85] W. Wieclawek and E. Pietka. Retina Analysis in Optical Coherence Tomography Images. In Information Technologies in Biomedicine, Volume 3, pages 247-258. Springer, 2014.

[86] G. Girish, A. Kothari, and J. Rajan. Automated Segmentation of Intra-Retinal Cysts from Optical Coherence Tomography Scans Using Marker Controlled Watershed Transform. In Engineering in Medicine and Biology Society (EMBC), pages 1292-1295. IEEE, 2016.

[87] W. Zhu, L. Zhang, F. Shi, D. Xiang, L. Wang, J. Guo, and at al. Automated Framework for Intraretinal Cystoid Macular Edema Segmentation in Three-Dimensional Optical Coherence Tomography Images with Macular Hole. Journal of Biomedical Optics, 22(7):076014, 2017.

[88] J. de Moura, J. Novo, J. Rouco, M. Penedo, and M. Ortega. Automatic Identification of Intraretinal Cystoid Regions in Optical Coherence Tomography. In Conference on Artificial Intelligence in Medicine in Europe, pages 305-315. Springer, 2017.

[89] Abdolreza Rashno, Dara D Koozekanani, Paul M Drayna, Behzad Nazari, Saeed Sadri, Hossein Rabbani, and Keshab K Parhi. Fully-Automated Segmentation of Fluid/Cyst Regions in Optical Coherence Tomography Images with Diabetic Macular Edema Using Neutrosophic Sets and Graph Algorithms. IEEE Transactions on Biomedical Engineering, 2017.

[90] Karthik Gopinath and Jayanthi Sivaswamy. Segmentation of Retinal Cysts from Optical Coherence Tomography Volumes Via Selective Enhancement. IEEE Journal of Biomedical and Health Informatics, 2018.

[91] G Girish, Bibhash Thakur, Sohini Roy Chowdhury, Abhishek Kothari, and Jeny Rajan. Segmentation of Intra-Retinal Cysts from Optical Coherence Tomography Images Using a Fully Convolutional Neural Network Model. IEEE Journal of Biomedical and Health Informatics, 2018. 
[92] G. Samagaio, J. de Moura, J. Novo, and M. Ortega. Optical Coherence Tomography Denoising by Means of a Fourier Butterworth Filter-Based Approach. In International Conference on Image Analysis and Processing, pages 422-432. Springer, 2017.

[93] S. Chiu, X. Li, P. Nicholas, C. Toth, J. Izatt, and S. Farsiu. Automatic segmentation of Seven Retinal Layers in SDOCT Images Congruent with Expert Manual Segmentation. Optics Express, 18(18):19413-19428, 2010.

[94] E. Dijkstra. A Note on Two Problems in Connexion with Graphs. Numerische Mathematik, 1(1):269-271, 1959.

[95] S. Zhu and A. Yuille. Region Competition: Unifying Snakes, Region Growing, and Bayes/MDL for Multiband Image Segmentation. IEEE Transactions on Pattern Analysis and Machine Intelligence, 18(9):884-900, 1996.

[96] T. Murakami and N. Yoshimura. Structural Changes in Individual Retinal Layers in Diabetic Macular Edema. Journal of Diabetes Research, 2013, 2013.

[97] J. Gelfand, R. Nolan, D. Schwartz, J. Graves, and A. Green. Microcystic Macular Oedema in Multiple Sclerosis is Associated with Disease Severity. Brain, 135(6):1786-1793, 2012.

[98] N. Otsu. A Threshold Selection Method from Gray-Level Histograms. IEEE Transactions on Systems, Man, and Cybernetics, 9(1):62-66, 1979.

[99] H. Noma, H. Funatsu, T. Mimura, and K. Shimada. Visual Function and Serous Retinal Detachment in Patients with Branch Retinal Vein Occlusion and Macular Edema: A Case Series. BMC Ophthalmology, 11(1):29, 2011.

[100] A. Gupta, R. Raman, K. Mohana, V. Kulothungan, and T. Sharma. Communications Between Intraretinal and Subretinal Space on Optical Coherence Tomography of Neurosensory Retinal Detachment in Diabetic Macular Edema. Oman Journal of Ophthalmology, 6(3):183, 2013.

[101] L. Carmona-Moxica and F. Hernández-Núñez. Revisión Bibliográfica: Edema Macular Diabético, Repercusiones y ratamiento. Revista Médica del Instituto Mexicano del Seguro Social, 53(5):600-607, 2015.

[102] S. Ooto, A. Tsujikawa, S. Mori, H. Tamura, K. Yamashiro, and N. Yoshimura. Thickness of Photoreceptor Layers in Polypoidal Choroidal Vasculopathy and Central Serous Chorioretinopathy. Graefe's Archive for Clinical and Experimental Ophthalmology, 248(8):1077-1086, 2010.

[103] R. Haralick, K. Shanmugam, et al. Textural Features for Image Classification. IEEE Transactions on Systems, Man, and Cybernetics, SMC-3(6):610-621, 1973.

[104] W. Siedlecki and J. Sklansky. On Automatic Feature Selection. In Handbook of Pattern Recognition and Computer Vision, pages 63-87. World Scientific, 1993.

[105] Feiping Nie, Heng Huang, Xiao Cai, and Chris H Ding. Efficient and Robust Feature Selection Via Joint $\ell 2,1$-norms Minimization. In Advances in Neural Information Processing Systems, pages 1813-1821, 2010. 
[106] Jinbo Bi, Kristin Bennett, Mark Embrechts, Curt Breneman, and Minghu Song. Dimensionality Reduction Via Sparse Support Vector Machines. Journal of Machine Learning Research,3(Mar):1229-1243, 2003.

[107] N. Friedman, D. Geiger, and M. Goldszmidt. Bayesian Network Classifiers. Machine Learning, 29(2-3):131-163, 1997.

[108] C. Cortes and V. Vapnik. Support-Vector Networks. Machine Learning, 20(3):273-297, 1995.

[109] Perseus. Classification Parameter Optimization, 2015. Disponível em http://www.coxdocs.org/doku.php?id=perseus:user:activities: matrixprocessing:learning:classificationparameteroptimization.

[110] J. de Moura, J. Novo, P. Charlón, N. Barreira, and M. Ortega. Enhanced Visualization of the Retinal Vasculature Using Depth Information in OCT. Medical \& Biological Engineering \& Computing, 55(12):2209-2225, 2017.

[111] H. Hahn and H. Peitgen. Iwt-Interactive Watershed Transform: A Hierarchical Method for Efficient Interactive and Automated Segmentation of Multidimensional Gray-Scale Images. In Medical Imaging 2003: Image Processing, volume 5032, pages 643-654. International Society for Optics and Photonics, 2003.

[112] B. Wolff, G. Azar, V. Vasseur, J. Sahel, C. Vignal, and M. Mauget-Faÿsse. Microcystic Changes in the Retinal Internal Nuclear ILayer Associated with Optic Atrophy: A Prospective Study. Journal of Ophthalmology, 2014, 2014.

[113] Y. Helmy and H. Allah. Optical Coherence Tomography Classification of Diabetic Cystoid Macular Edema. Clinical Ophthalmology, 7:1731, 2013.

[114] W. Goebel and T.a Kretzchmar-Gross. Retinal Thickness in Diabetic Retinopathy: A Study Using Optical Coherence Tomography (OCT). Retina, 22(6):759-767, 2002.

[115] Kiran Vupparaboina, Srinath Nizampatnam, Jay Chhablani, Ashutosh Richhariya, and Soumya Jana. Automated Estimation of Choroidal Thickness Distribution and Volume Based on OCT Images of Posterior Visual Section. Computerized Medical Imaging and Graphics, 46:315-327, 2015.

[116] Gabriela Samagaio, AiAda Estévez, Joaquim de Moura, Jorge Novo, Maria Isabel Fernandez, and Marcos Ortega. Automatic Macular Edema Identification and Characterization Using OCT Images. Computer Methods and Programs in Biomedicine, 2018. 